\title{
SEARCH FOR SPATIALLY EXTENDED FERMI LARGE AREA TELESCOPE SOURCES USING TWO YEARS OF DATA
}

\author{
J. Lande ${ }^{1}$, M. Ackermann ${ }^{2}$, A. Allafort ${ }^{1}$, J. Ballet ${ }^{3}$, K. Bechtol ${ }^{1}$, T. H. BurnetT ${ }^{4}$, J. Cohen-Tanugi ${ }^{5}$, \\ A. Drlica-Wagner ${ }^{1}$, S. Funk ${ }^{1}$, F. Giordano ${ }^{6,7}$, M.-H. Grondin ${ }^{8,9}$, M. KerR $^{1}$, And M. Lemoine-Goumard ${ }^{10,11}$ \\ ${ }^{1}$ W. W. Hansen Experimental Physics Laboratory, Kavli Institute for Particle Astrophysics and Cosmology, Department of Physics and SLAC National Accelerator \\ Laboratory, Stanford University, Stanford, CA 94305, USA; joshualande@ gmail.com, funk@ slac.stanford.edu \\ ${ }^{2}$ Deutsches Elektronen Synchrotron DESY, D-15738 Zeuthen, Germany; markus.ackermann@ desy.de \\ ${ }^{3}$ Laboratoire AIM, CEA-IRFU/CNRS/Université Paris Diderot, Service d'Astrophysique, CEA Saclay, F-91191 Gif sur Yvette, France \\ ${ }^{4}$ Department of Physics, University of Washington, Seattle, WA 98195-1560, USA \\ ${ }^{5}$ Laboratoire Univers et Particules de Montpellier, Université Montpellier 2, CNRS/IN2P3, F-34095 Montpellier, France \\ ${ }^{6}$ Dipartimento di Fisica “M. Merlin” dell’Università e del Politecnico di Bari, I-70126 Bari, Italy \\ ${ }^{7}$ Istituto Nazionale di Fisica Nucleare, Sezione di Bari, I-70126 Bari, Italy \\ ${ }^{8}$ Max-Planck-Institut für Kernphysik, D-69029 Heidelberg, Germany \\ ${ }^{9}$ Landessternwarte, Universität Heidelberg, Königstuhl, D-69117 Heidelberg, Germany \\ ${ }^{10}$ Centre d'Études Nucléaires de Bordeaux Gradignan, Université Bordeaux 1, CNRS/IN2p3, F-33175 Gradignan, France \\ Received 2011 December 19; accepted 2012 June 29; published 2012 August 8
}

\begin{abstract}
Spatial extension is an important characteristic for correctly associating $\gamma$-ray-emitting sources with their counterparts at other wavelengths and for obtaining an unbiased model of their spectra. We present a new method for quantifying the spatial extension of sources detected by the Large Area Telescope (LAT), the primary science instrument on the Fermi Gamma-ray Space Telescope (Fermi). We perform a series of Monte Carlo simulations to validate this tool and calculate the LAT threshold for detecting the spatial extension of sources. We then test all sources in the second Fermi-LAT catalog for extension. We report the detection of seven new spatially extended sources.
\end{abstract}

Key words: catalogs - gamma rays: general - ISM: supernova remnants - methods: statistical

Online-only material: color figures

\section{INTRODUCTION}

A number of astrophysical source classes including supernova remnants (SNRs), pulsar wind nebulae (PWNe), molecular clouds, normal galaxies, and galaxy clusters are expected to be spatially resolvable by the Large Area Telescope (LAT), the primary instrument on the Fermi Gamma-ray Space Telescope (Fermi). Additionally, dark matter satellites are also hypothesized to be spatially extended. See Atwood et al. (2009) for prelaunch predictions. The LAT has detected seven SNRs which are significantly extended at $\mathrm{GeV}$ energies: W51C, W30, IC 443, W28, W44, RX J1713.7-3946, and the Cygnus Loop (Abdo et al. 2009a, 2010f, 2010h, 2010i, 2011, 2012b; Katagiri et al. 2011). In addition, three extended PWNe have been detected by the LAT: MSH 15-52, Vela X, and HESS J1825-137 (Abdo et al. 2010b, 2010g; Grondin et al. 2011). Two nearby galaxies, the Large Magellanic Cloud (LMC) and Small Magellanic Cloud (SMC), and the lobes of one radio galaxy, Centaurus A, were spatially resolved at $\mathrm{GeV}$ energies (Abdo et al. 2010c, $2010 \mathrm{~d}, 2010 \mathrm{j})$. A number of additional sources detected at $\mathrm{GeV}$ energies are positionally coincident with sources that exhibit large enough extension at other wavelengths to be spatially resolvable by the $\mathrm{LAT}$ at $\mathrm{GeV}$ energies. In particular, there are $59 \mathrm{GeV}$ sources in the second Fermi Source Catalog (2FGL) that might be associated with extended SNRs (2FGL; Nolan et al. 2012). Previous analyses of extended LAT sources were performed as dedicated studies of individual sources so we expect that a systematic scan of all LAT-detected sources could uncover additional spatially extended sources.

\footnotetext{
${ }^{11}$ Funded by contract ERC-StG-259391 from the European Community.
}

The current generation of air Cerenkov detectors has made it apparent that many sources can be spatially resolved at even higher energies. Most prominent was a survey of the Galactic plane using the High Energy Stereoscopic System (H.E.S.S.) which reported 14 spatially extended sources with extensions varying from $\sim 0.1$ to $\sim 0.25$ (Aharonian et al. 2006d). Within our Galaxy very few sources detected at $\mathrm{TeV}$ energies, most notably the $\gamma$-ray binaries LS 5039 (Aharonian et al. 2006a), LS I+61-303 (Albert et al. 2006; Acciari et al. 2011), HESS J0632+057 (Aharonian et al. 2007c), and the Crab Nebula (Weekes et al. 1989), have no detectable extension. High-energy $\gamma$-rays from $\mathrm{TeV}$ sources are produced by the decay of $\pi^{0}$ s produced by hadronic interactions with interstellar matter and by relativistic electrons due to inverse Compton (IC) scattering and bremsstrahlung radiation. It is plausible that the $\mathrm{GeV}$ and $\mathrm{TeV}$ emission from these sources originates from the same population of high-energy particles and so at least some of these sources should be detectable at $\mathrm{GeV}$ energies. Studying these $\mathrm{TeV}$ sources at $\mathrm{GeV}$ energies would help to determine the emission mechanisms producing these high-energy photons.

The LAT is a pair conversion telescope that has been surveying the $\gamma$-ray sky since 2008 August. The LAT has broad energy coverage ( $20 \mathrm{MeV}$ to $>300 \mathrm{GeV})$, wide field of view $(\sim 2.4 \mathrm{sr})$, and large effective area $\left(\sim 8000 \mathrm{~cm}^{2}\right.$ at $\left.>1 \mathrm{GeV}\right)$. Additional information about the performance of the LAT can be found in Atwood et al. (2009).

Using two years of all-sky survey data, the LAT Collaboration published 2FGL (Nolan et al. 2012). The possible counterparts of many of these sources can be spatially resolved when observed at other frequencies. But detecting the spatial extension of these sources at $\mathrm{GeV}$ energies is difficult because the size of 
the point-spread function (PSF) of the LAT is comparable to the typical size of many of these sources.

The capability to spatially resolve GeV $\gamma$-ray sources is important for several reasons. Finding a coherent source extension across different energy bands can help to associate a LAT source to an otherwise confused counterpart. Furthermore, $\gamma$-ray emission from dark matter annihilation has been predicted to be detectable by the LAT. Some of the dark matter substructure in our Galaxy could be spatially resolvable by the LAT (Baltz et al. 2008). Characterization of spatial extension could help to identify this substructure. Also, due to the strong energy dependence of the LAT PSF, the spatial and spectral characterization of a source cannot be decoupled. An inaccurate spatial model will bias the spectral model of the source and vice versa. Specifically, modeling a spatially extended source as point-like will systematically soften measured spectra. Furthermore, correctly modeling source extension is important for understanding an entire region of the sky. For example, an imperfect model of the spatially extended LMC introduced significant residuals in the surrounding region (Abdo et al. 2010e; Nolan et al. 2012). Such residuals can bias the significance and measured spectra of neighboring sources in the densely populated Galactic plane.

For these reasons, in Section 2 we present a new systematic method for analyzing spatially extended LAT sources. In Section 3, we demonstrate that this method can be used to test the statistical significance of the extension of a LAT source and we assess the expected level of bias introduced by assuming an incorrect spatial model. In Section 4, we calculate the LAT detection threshold to resolve the extension of a source. In Section 5, we study the ability of the LAT to distinguish between a single extended source and unresolved closely spaced point-like sources. In Section 6, we further demonstrate that our detection method does not misidentify point-like sources as being extended by testing the extension of active galactic nuclei (AGNs) believed to be unresolvable. In Section 7, we systematically reanalyze the 12 extended sources included in the 2FGL catalog and in Section 8 we describe a way to estimate systematic errors on the measured extension of a source. In Section 9, we describe a search for new spatially extended LAT sources. Finally, in Section 10 we present the detection of the extension of nine spatially extended sources that were reported in the 2FGL catalog but treated as point-like in the analysis. Two of these sources have been previously analyzed in dedicated publications.

\section{ANALYSIS METHODS}

Morphological studies of sources using the LAT are challenging because of the strongly energy-dependent PSF that is comparable in size to the extension of many sources expected to be detected at $\mathrm{GeV}$ energies. Additional complications arise for sources along the Galactic plane due to systematic uncertainties in the model for Galactic diffuse emission.

For energies below $\sim 300 \mathrm{MeV}$, the angular resolution is limited by multiple scattering in the silicon strip tracking section of the detector and is several degrees at $100 \mathrm{MeV}$. The PSF improves with energy approaching a $68 \%$ containment radius of $\sim 0.2$ at the highest energies (when averaged over the acceptance of the LAT) and is limited by the ratio of the strip pitch to the height of the tracker (Atwood et al. 2009; Abdo et al. 2009c, 2012a). ${ }^{12}$ However, since most

\footnotetext{
12 More information about the performance of the LAT can be found at the
} Fermi Science Support Center (FSSC, http://fermi.gsfc.nasa.gov). high-energy astrophysical sources have spectra that decrease rapidly with increasing energy, there are typically fewer higher energy photons with improved angular resolution. Therefore sophisticated analysis techniques are required to maximize the sensitivity of the LAT to extended sources.

\subsection{Modeling Extended Sources in the pointlike Package}

A new maximum likelihood analysis tool has been developed to address the unique requirements for studying spatially extended sources with the LAT. It works by maximizing the Poisson likelihood to detect the observed distributions of $\gamma$-rays (referred to as counts) given a parameterized spatial and spectral model of the sky. The data are binned spatially, using a HEALPix pixellization, and spectrally (Górski et al. 2005) and the likelihood is maximized over all bins in a region. The extension of a source can be modeled by a geometric shape (e.g., a disk or a two-dimensional (2D) Gaussian) and the position, extension, and spectrum of the source can be simultaneously fit.

This type of analysis is unwieldy using the standard LAT likelihood analysis tool gtlike ${ }^{13}$ because it can only fit the spectral parameters of the model unless a more sophisticated iterative procedure is used. We note that gtlike has been used in the past in several studies of source extension in the LAT Collaboration (Abdo et al. 2009a, 2010c, 2010f, 2010j). In these studies, a set of gtlike maximum likelihood fits at fixed extensions was used to build a profile of the likelihood as a function of extension. The gtlike likelihood profile approach has been shown to correctly reproduce the extension of simulated extended sources assuming that the true position is known (Giordano 2011). But it is not optimal because the position, extension, and spectrum of the source must be simultaneously fit to find the best-fit parameters and to maximize the statistical significance of the detection. Furthermore, because the gtlike approach is computationally intensive, no large-scale Monte Carlo simulations have been run to calculate its false detection rate.

The approach presented here is based on a second maximum likelihood fitting package developed in the LAT Collaboration called pointlike (Abdo et al. 2010e; Kerr 2011). The choice to base the spatial extension fitting on pointlike rather than gtlike was made due to considerations of computing time. The pointlike algorithm was optimized for speed to handle larger numbers of sources efficiently, which is important for our catalog scan and for being able to perform large-scale Monte Carlo simulations to validate the analysis. Details on the pointlike package can be found in Kerr (2011). We extended the code to allow a simultaneous fit of the source extension together with the position and the spectral parameters.

\subsection{Extension Fitting}

In pointlike, one can fit the position and extension of a source under the assumption that the source model can be factorized: $M(x, y, E)=S(x, y) \times X(E)$, where $S(x, y)$ is the spatial distribution and $X(E)$ is the spectral distribution. To fit an extended source, pointlike convolves the extended source shape with the PSF (as a function of energy) and uses the minuit library (James \& Roos 1975) to maximize the likelihood by simultaneously varying the position, extension, and spectrum of the source. As will be described in Section 3.1, simultaneously fitting the position, extension, and spectrum is important to

\footnotetext{
13 gtlike is distributed publicly by the FSSC.
} 
maximize the statistical significance of the detection of the extension of a source. To avoid projection effects, the longitude and latitude of the source are not directly fit but instead the displacement of the source in a reference frame centered on the source.

The significance of the extension of a source can be calculated from the likelihood-ratio test. The likelihood ratio defines the test statistic (TS) by comparing the likelihood of a simpler hypothesis to a more complicated one:

$$
\mathrm{TS}=2 \log \left(\mathcal{L}\left(H_{1}\right) / \mathcal{L}\left(H_{0}\right)\right)
$$

where $H_{1}$ is the more complicated hypothesis and $H_{0}$ the simpler one. For the case of the extension test, we compare the likelihood when assuming the source has either a point-like or spatially extended spatial model:

$$
\mathrm{TS}_{\text {ext }}=2 \log \left(\mathcal{L}_{\text {ext }} / \mathcal{L}_{\text {ps }}\right) .
$$

pointlike calculates $\mathrm{TS}_{\text {ext }}$ by fitting a source first with a spatially extended model and then as a point-like source. The interpretation of $\mathrm{TS}_{\mathrm{ext}}$ in terms of a statistical significance is discussed in Section 3.1.

For extended sources with an assumed radially symmetric shape, we optimized the calculation by performing one of the integrals analytically. The expected photon distribution can be written as

$$
\operatorname{PDF}(\boldsymbol{r})=\int \operatorname{PSF}\left(\left|\boldsymbol{r}-\boldsymbol{r}^{\prime}\right|\right) I_{\mathrm{src}}\left(\boldsymbol{r}^{\prime}\right) r^{\prime} d r^{\prime} d \phi^{\prime},
$$

where $\boldsymbol{r}$ represents the position in the sky and $I_{\mathrm{src}}(\boldsymbol{r})$ is the spatial distribution of the source. The PSF of the LAT is currently parameterized in the Pass 7_V6 (P7_V6) Source Instrument Response Functions (IRFs; Abdo et al. 2012a) by a King function (King 1962):

$$
\operatorname{PSF}(r)=\frac{1}{2 \pi \sigma^{2}}\left(1-\frac{1}{\gamma}\right)\left(1+\frac{u}{\gamma}\right)^{-\gamma},
$$

where $u=(r / \sigma)^{2} / 2$ and $\sigma$ and $\gamma$ are free parameters (Kerr 2011). For radially symmetric extended sources, the angular part of the integral can be evaluated analytically

$$
\begin{aligned}
\operatorname{PDF}(u)= & \int_{0}^{\infty} r^{\prime} d r^{\prime} I_{\mathrm{src}}(v) \int_{0}^{2 \pi} d \phi^{\prime} \mathrm{PSF} \\
& \times\left(\sqrt{2 \sigma^{2}\left(u+v-2 \sqrt{u v} \cos \left(\phi-\phi^{\prime}\right)\right)}\right) \\
= & \int_{0}^{\infty} d v I_{\mathrm{src}}(v)\left(\frac{\gamma-1}{\gamma}\right)\left(\frac{\gamma}{\gamma+u+v}\right)^{\gamma} \\
& \times{ }_{2} F_{1}\left(\gamma / 2, \frac{1+\gamma}{2}, 1, \frac{4 u v}{(\gamma+u+v)^{2}}\right)
\end{aligned}
$$

where $v=\left(r^{\prime} / \sigma\right)^{2} / 2$ and ${ }_{2} F_{1}$ is the Gaussian hypergeometric function. This convolution formula reduces the expected photon distribution to a single numerical integral.

There will always be a small numerical discrepancy between the expected photon distribution derived from a true point-like source and a very small extended source due to numerical error in the convolution. In most situations, this error is insignificant. But in particular for very bright sources, this numerical error has the potential to bias the TS for the extension test. Therefore, when calculating $\mathrm{TS}_{\text {ext }}$, we compare the likelihood fitting the source with an extended spatial model to the likelihood when the extension is fixed to a very small value $\left(10^{-10} \mathrm{deg}\right.$ in radius for a uniform disk model).

We estimate the error on the extension of a source by fixing the position of the source and varying the extension until the log of the likelihood has decreased by $1 / 2$, corresponding to a $1 \sigma$ error (Eadie et al. 1971). Figure 1 demonstrates this method by showing the change in the log of the likelihood when varying the modeled extension of the SNR IC 443. The localization error is calculated by fixing the extension and spectrum of the source to their best-fit values and then fitting the log of the likelihood to a 2D Gaussian as a function of position. This localization error algorithm is further described in Nolan et al. (2012).

\section{3. gtlike Analysis Validation}

pointlike is important for analyses of LAT data that require many iterations such as source localization and extension fitting. On the other hand, because gtlike makes fewer approximations in calculating the likelihood we expect the spectral parameters found with gtlike to be slightly more accurate. Furthermore, because gtlike is the standard likelihood analysis package for LAT data, it has been more extensively validated for spectral analysis. For those reasons, in the following analysis we used pointlike to determine the position and extension of a source and subsequently derived the spectrum using gtlike. Both gtlike and pointlike can be used to estimate the statistical significance of the extension of a source and we required that both methods agree for a source to be considered extended. There was good agreement between the two methods. Unless explicitly mentioned, all $\mathrm{TS}, \mathrm{TS}_{\mathrm{ext}}$, and spectral parameters were calculated using gtlike with the best-fit positions and extension found by pointlike.

\subsection{Comparing Source Sizes}

We considered two models for the surface brightness profile for extended sources: a 2D Gaussian model

$$
I_{\text {Gaussian }}(x, y)=\frac{1}{2 \pi \sigma^{2}} \exp \left(-\left(x^{2}+y^{2}\right) / 2 \sigma^{2}\right),
$$

or a uniform disk model

$$
I_{\text {disk }}(x, y)= \begin{cases}\frac{1}{\pi \sigma^{2}} & x^{2}+y^{2} \leqslant \sigma^{2} \\ 0 & x^{2}+y^{2}>\sigma^{2} .\end{cases}
$$

Although these shapes are significantly different, Figure 2 shows that, after convolution with the LAT PSF, their probability density functions (PDFs) are similar for a source that has a 0.5 radius typical of LAT-detected extended sources. To allow a valid comparison between the Gaussian and the uniform disk models, we define the source size as the radius containing $68 \%$ of the intensity $\left(r_{68}\right)$. By direct integration, we find

$$
\begin{gathered}
r_{68, \text { Gaussian }}=1.51 \sigma, \\
r_{68, \text { disk }}=0.82 \sigma,
\end{gathered}
$$

where $\sigma$ is defined in Equations (7) and (8), respectively. For the example above, $r_{68}=0.5$ so $\sigma_{\text {disk }}=0.61$ and $\sigma_{\text {Gaussian }}=0.33$.

For sources that are comparable in size to the PSF, the differences in the PDF for different spatial models are lost in the 

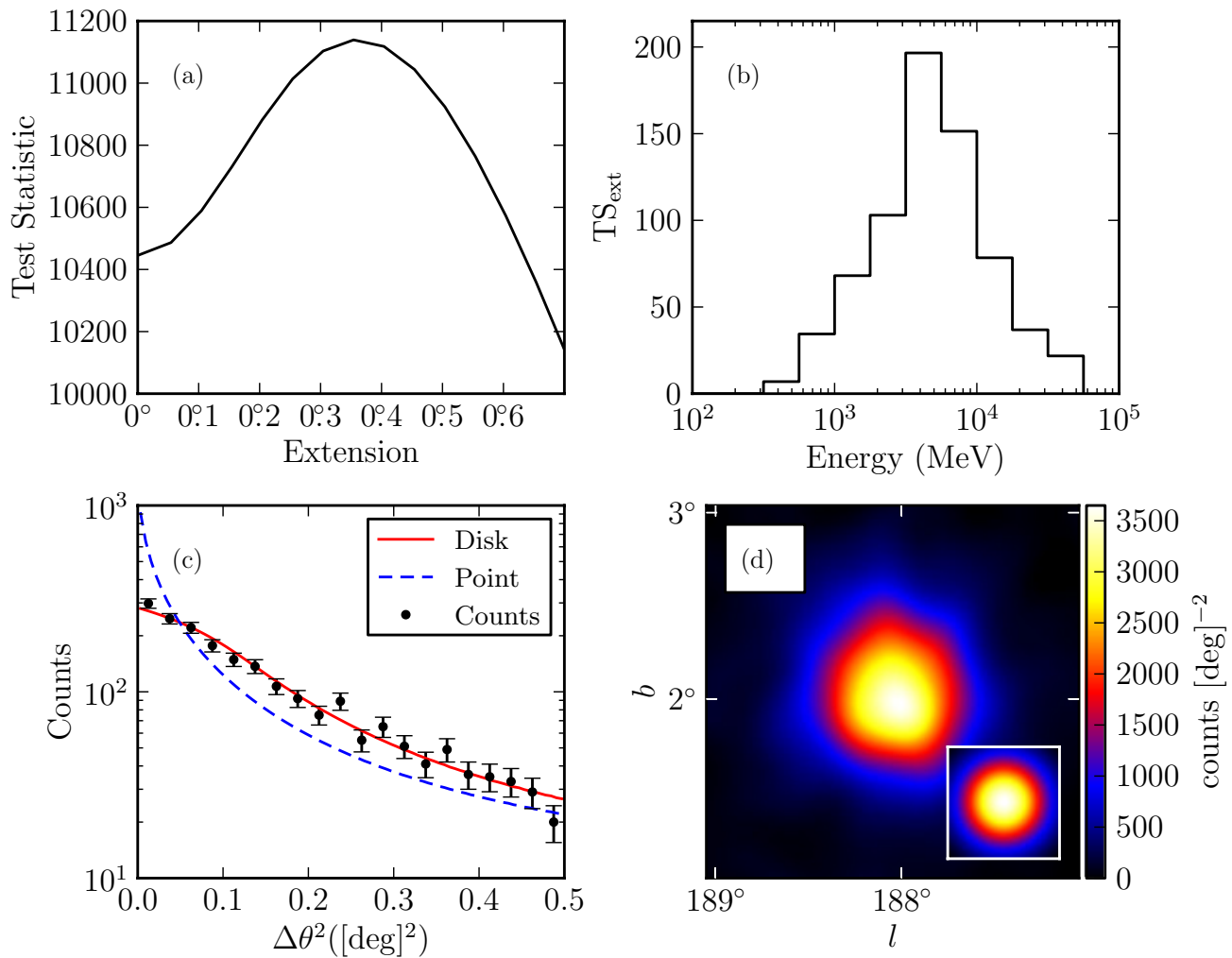

Figure 1. Counts maps and TS profiles for the SNR IC 443. (a) TS vs. extension of the source. (b) $\mathrm{TS}_{\text {ext }}$ for individual energy bands. (c) Observed radial profile of counts in comparison to the expected profiles for a spatially extended source (solid and colored red in the online version) and for a point-like source (dashed and colored blue in the online version). (d) Smoothed counts map after subtraction of the diffuse emission compared to the smoothed LAT PSF (inset). Both were smoothed by a $0.12 \mathrm{D}$ Gaussian kernel. Plots (a), (c), and (d) use only photons with energies between $1 \mathrm{GeV}$ and $100 \mathrm{GeV}$. Plots (c) and (d) include only photons which converted in the front part of the tracker and have an improved angular resolution (Atwood et al. 2009).

(A color version of this figure is available in the online journal.)

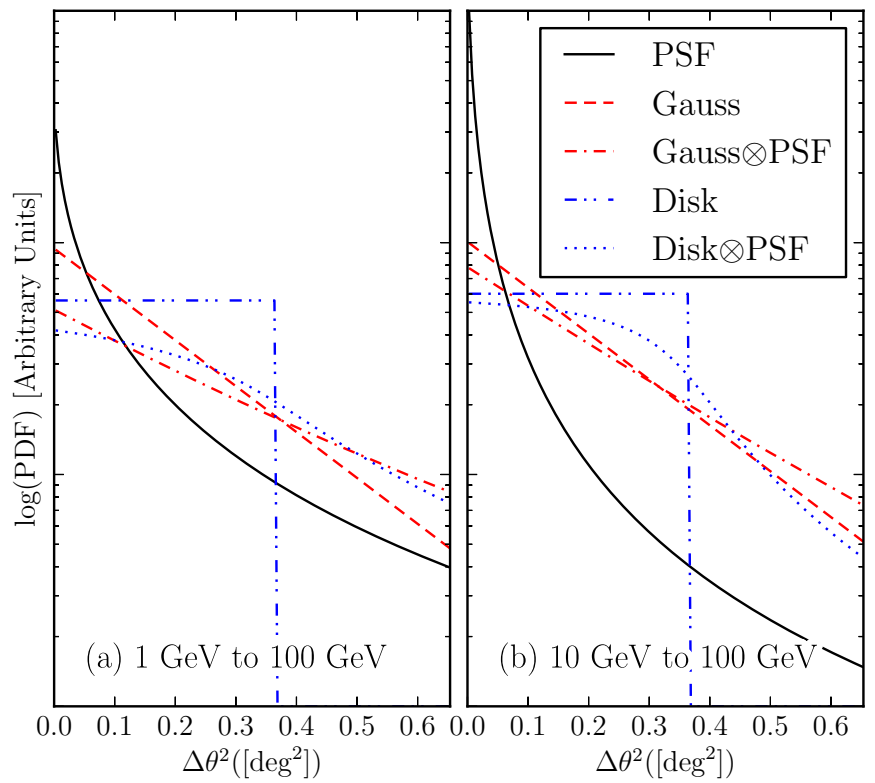

Figure 2. Comparison of a 2D Gaussian and uniform disk spatial model of extended sources before and after convolving with the PSF for two energy ranges. The solid black line is the PSF that would be observed for a power-law source of spectral index 2. The dashed line and the dash-dotted lines are the brightness profile of a Gaussian with $r_{68}=0.5$ and the convolution of this profile with the LAT PSF, respectively (colored red in the online version). The dash-dot-dotted and the dot-dotted lines are the brightness profile of a uniform disk with $r_{68}=0.5$ and the convolution of this profile with the LAT PSF respectively (colored blue in the online version).

(A color version of this figure is available in the online journal.) noise and the LAT is not sensitive to the detailed spatial structure of these sources. In Section 3.3, we perform a dedicated Monte Carlo simulation that shows there is little bias due to incorrectly modeling the spatial structure of an extended source. Therefore, in our search for extended sources we use only a radially symmetric uniform disk spatial model. Unless otherwise noted, we quote the radius to the edge $(\sigma)$ as the size of the source.

\section{VALIDATION OF THE TS DISTRIBUTION}

\subsection{Point-like Source Simulations Over a Uniform Background}

We tested the theoretical distribution for $\mathrm{TS}_{\text {ext }}$ to evaluate the false detection probability for measuring source extension. To do so, we tested simulated point-like sources for extension. Mattox et al. (1996) discuss that the TS distribution for a likelihood-ratio test on the existence of a source at a given position is

$$
P(\mathrm{TS})=\frac{1}{2}\left(\chi_{1}^{2}(\mathrm{TS})+\delta(\mathrm{TS})\right)
$$

where $P(\mathrm{TS})$ is the probability density to get a particular value of TS, $\chi_{1}^{2}$ is the chi-squared distribution with one degree of freedom, and $\delta$ is the Dirac delta function. The particular form of Equation (11) is due to the null hypothesis (source flux $\Phi=0$ ) residing on the edge of parameter space and the model hypothesis adding a single degree of freedom (the source flux). It leads to the often quoted result $\sqrt{\mathrm{TS}}=\sigma$, where $\sigma$ here refers to the significance of the detection. It is plausible to expect a similar distribution for the TS in the test for source extension since the same conditions apply (with the source flux $\Phi$ replaced 
Table 1

Monte Carlo Spectral Parameters

\begin{tabular}{|c|c|c|c|c|c|}
\hline Spectral Index & $\begin{array}{c}\text { Flux }^{\mathrm{a}} \\
\left(\mathrm{ph} \mathrm{cm}^{-2} \mathrm{~s}^{-1}\right)\end{array}$ & $N_{1-100 \mathrm{GeV}}$ & $\langle\mathrm{TS}\rangle_{1-100 \mathrm{GeV}}$ & $N_{10-100 \mathrm{GeV}}$ & $\langle\mathrm{TS}\rangle_{10-100 \mathrm{GeV}}$ \\
\hline \multicolumn{6}{|c|}{ Isotropic background } \\
\hline 1.5 & $\begin{array}{r}3 \times 10^{-7} \\
10^{-7} \\
3 \times 10^{-8} \\
10^{-8} \\
3 \times 10^{-9} \\
\end{array}$ & $\begin{array}{l}18938 \\
19079 \\
19303 \\
19385 \\
18694 \\
\end{array}$ & $\begin{array}{r}22233 \\
5827 \\
1276 \\
303 \\
62 \\
\end{array}$ & $\begin{array}{l}18938 \\
19079 \\
19303 \\
19381 \\
12442 \\
\end{array}$ & $\begin{array}{r}8084 \\
2258 \\
541 \\
142 \\
43 \\
\end{array}$ \\
\hline 2 & $\begin{array}{r}10^{-6} \\
3 \times 10^{-7} \\
10^{-7} \\
3 \times 10^{-8} \\
10^{-8}\end{array}$ & $\begin{array}{l}18760 \\
18775 \\
18804 \\
18836 \\
17060\end{array}$ & $\begin{array}{r}22101 \\
4913 \\
1170 \\
224 \\
50\end{array}$ & $\begin{array}{c}18760 \\
18775 \\
18803 \\
15256 \\
\ldots\end{array}$ & $\begin{array}{r}3033 \\
730 \\
192 \\
50 \\
\ldots\end{array}$ \\
\hline 2.5 & $\begin{array}{r}3 \times 10^{-6} \\
10^{-6} \\
3 \times 10^{-7} \\
10^{-7} \\
3 \times 10^{-8}\end{array}$ & $\begin{array}{l}18597 \\
18609 \\
18613 \\
18658 \\
14072 \\
\end{array}$ & $\begin{array}{r}19036 \\
4738 \\
954 \\
203 \\
41 \\
\end{array}$ & $\begin{array}{c}18597 \\
18608 \\
15958 \\
\ldots \\
\ldots \\
\end{array}$ & $\begin{array}{r}786 \\
208 \\
53 \\
\ldots \\
\ldots \\
\end{array}$ \\
\hline 3 & $\begin{array}{r}10^{-5} \\
3 \times 10^{-6} \\
10^{-6} \\
3 \times 10^{-7} \\
10^{-7} \\
\end{array}$ & $\begin{array}{l}18354 \\
18381 \\
18449 \\
18517 \\
13714 \\
\end{array}$ & $\begin{array}{r}19466 \\
4205 \\
966 \\
174 \\
41 \\
\end{array}$ & $\begin{array}{c}18354 \\
15973 \\
\ldots \\
\ldots \\
\ldots \\
\end{array}$ & $\begin{array}{r}215 \\
54 \\
\ldots \\
\ldots \\
\ldots \\
\end{array}$ \\
\hline \multicolumn{6}{|c|}{ Galactic diffuse and isotropic background ${ }^{\mathrm{b}}$} \\
\hline $\begin{array}{l}1.5 \\
2 \\
2.5 \\
3\end{array}$ & $\begin{array}{l}2.3 \times 10^{-8} \\
1.2 \times 10^{-7} \\
4.5 \times 10^{-7} \\
2.0 \times 10^{-6}\end{array}$ & $\begin{array}{l}90741 \\
92161 \\
86226 \\
94412\end{array}$ & $\begin{array}{l}63 \\
60 \\
47 \\
61\end{array}$ & $\begin{array}{l}\ldots \\
\ldots \\
\ldots \\
\ldots\end{array}$ & $\begin{array}{l}\ldots \\
\ldots \\
\ldots \\
\ldots\end{array}$ \\
\hline
\end{tabular}

Notes. A list of the spectral models of the simulated point-like sources which were tested for extension. For each model, the number of statistically independent simulations and the average value of TS is also tabulated. The top rows are the simulations on top of an isotropic background and the bottom rows are the simulations on top of the Galactic diffuse and isotropic background.

${ }^{\text {a }}$ Integral $100 \mathrm{MeV}$ to $100 \mathrm{GeV}$ flux.

${ }^{b}$ For the Galactic simulations, the quoted fluxes are the fluxes for sources placed in the Galactic center. The actual fluxes are scaled by Equation (12).

by the source radius $r$ and $r<0$ being unphysical). To verify Equation (11), we evaluated the empirical distribution function of $\mathrm{TS}_{\mathrm{ext}}$ computed from simulated sources.

We simulated point-like sources with various spectral forms using the LAT on-orbit simulation tool gtobssim ${ }^{14}$ and fit the sources with pointlike using both point-like and extended source hypotheses. These point-like sources were simulated with a power-law spectral model with integrated fluxes above $100 \mathrm{MeV}$ ranging from $3 \times 10^{-9}$ to $1 \times 10^{-5} \mathrm{ph} \mathrm{cm}^{-2} \mathrm{~s}^{-1}$ and spectral indices ranging from 1.5 to 3 . These values were picked to represent typical parameters of LAT-detected sources. The point-like sources were simulated on top of an isotropic background with a power-law spectral model with integrated flux above $100 \mathrm{MeV}$ of $1.5 \times 10^{-5} \mathrm{ph} \mathrm{cm}^{-2} \mathrm{~s}^{-1} \mathrm{sr}^{-1}$ and spectral index 2.1. This was taken to be the same as the isotropic spectrum measured by EGRET (Sreekumar et al. 1998). This spectrum is comparable to the high-latitude background intensity seen by the LAT. The Monte Carlo simulation was performed over a one-year observation period using a representative spacecraft orbit and livetime. The reconstruction was performed using the P7_V6 Source class event selection and IRFs (Abdo et al. 2012a). For each significantly detected pointlike source ( $T S \geqslant 25$ ), we used pointlike to fit the source as

\footnotetext{
14 gtobssim is distributed publicly by the FSSC.
}

an extended source and calculate $\mathrm{TS}_{\text {ext }}$. This entire procedure was performed twice, once fitting in the $1-100 \mathrm{GeV}$ energy range and once fitting in the $10-100 \mathrm{GeV}$ energy range.

For each set of spectral parameters, $\sim 20,000$ statistically independent simulations were performed. For lower-flux spectral models, many of the simulations left the source insignificant $(\mathrm{TS}<25)$ and were discarded. Table 1 shows the different spectral models used in our study as well as the number of simulations and the average point-like source significance. The cumulative density of $\mathrm{TS}_{\text {ext }}$ is plotted in Figures 3 and 4 and compared to the $\chi_{1}^{2} / 2$ distribution of Equation (11).

Our study shows broad agreement between simulations and Equation (11). To the extent that there is a discrepancy, the simulations tended to produce smaller than expected values of $\mathrm{TS}_{\text {ext }}$ which would make the formal significance conservative. Considering the distribution in Figures 3 and 4, the choice of a threshold $\mathrm{TS}_{\text {ext }}$ set to 16 (corresponding to a formal $4 \sigma$ significance) is reasonable.

\subsection{Point-like Source Simulations over a Structured Background}

We performed a second set of simulations to show that the theoretical distribution for $\mathrm{TS}_{\mathrm{ext}}$ is still preserved when the point-like sources are present over a highly structured diffuse 


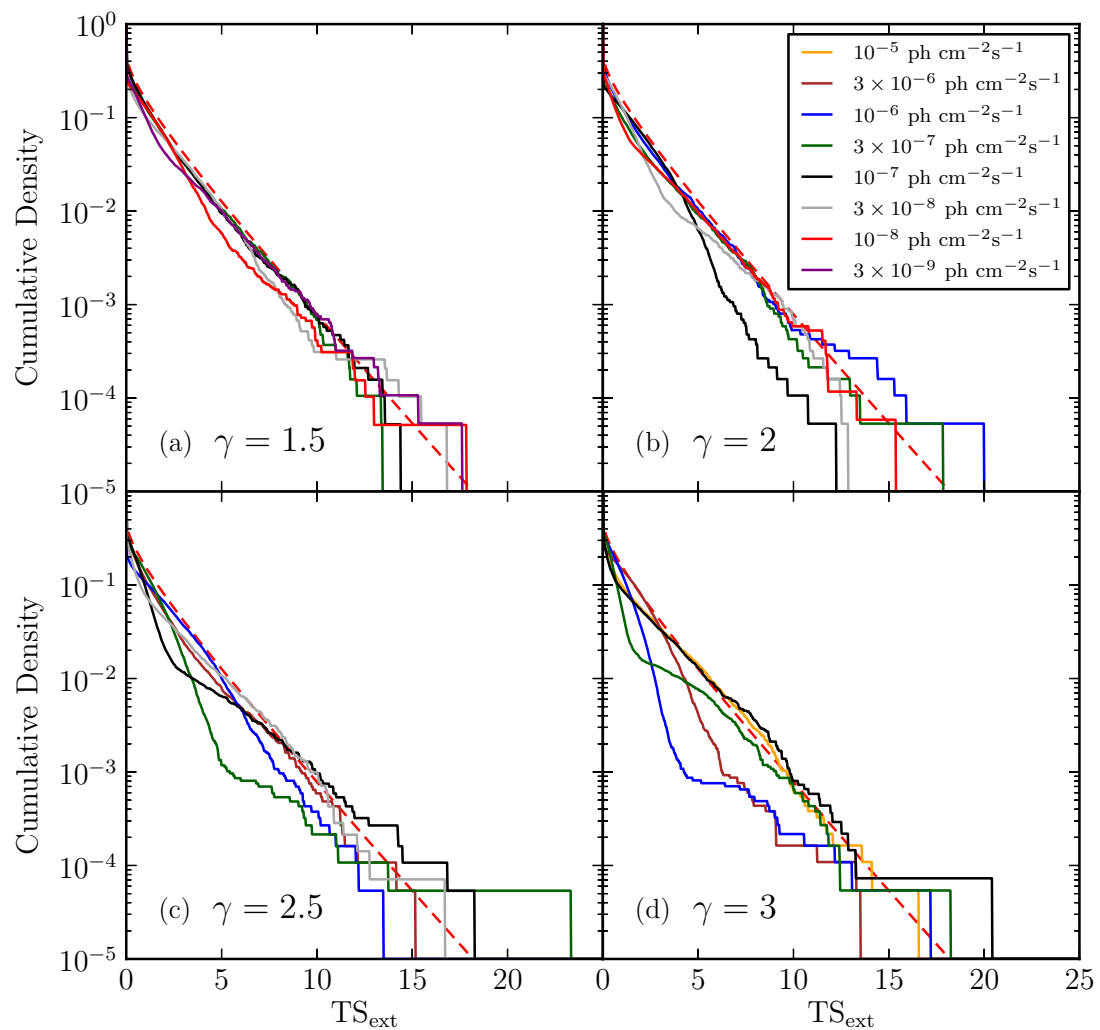

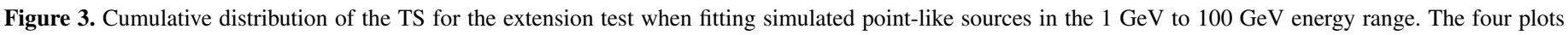

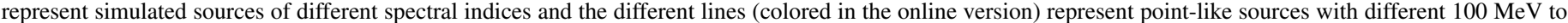
$100 \mathrm{GeV}$ integral fluxes. The dashed line (colored red) is the cumulative density function of Equation (11).

(A color version of this figure is available in the online journal.)

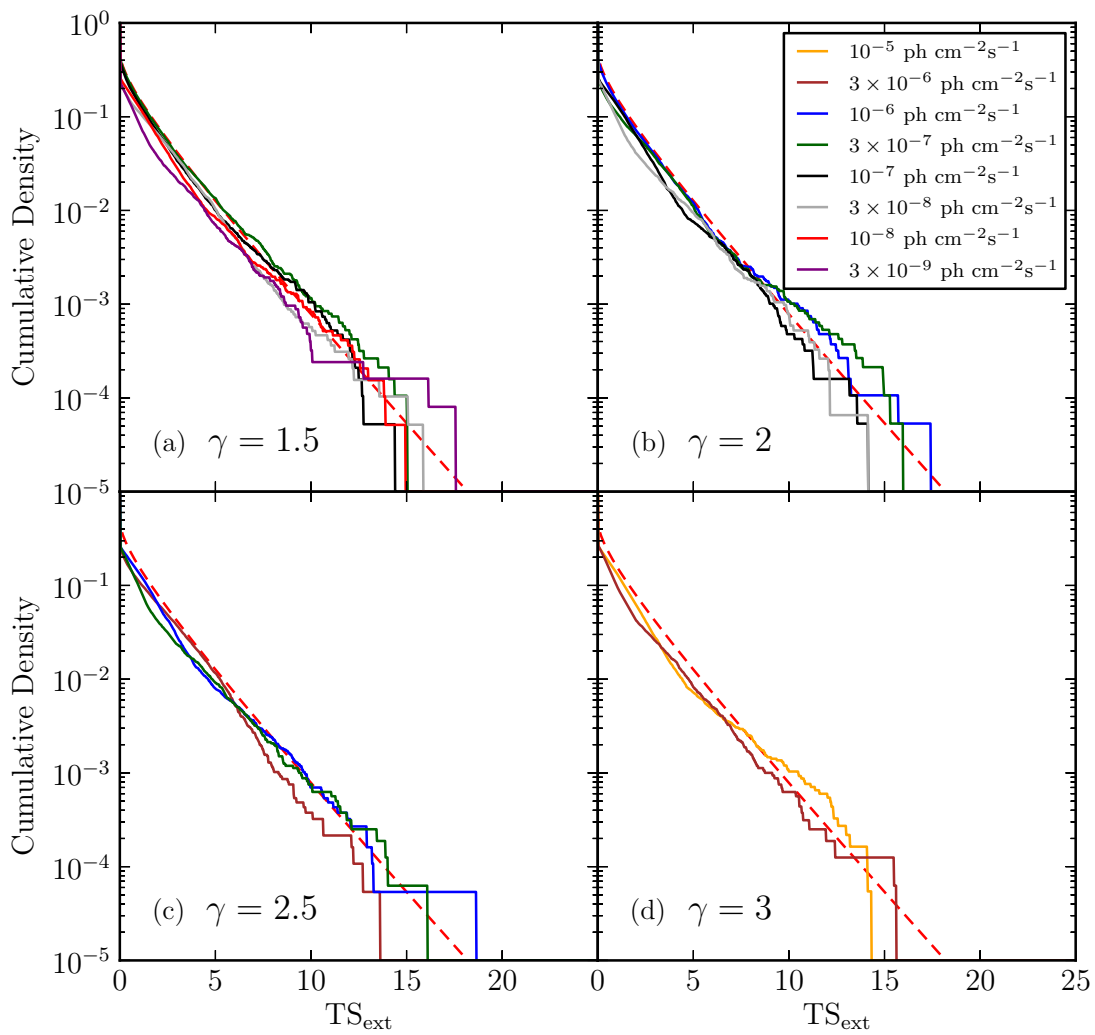

Figure 4. Same plot as Figure 3 but fitting in the 10-100 GeV energy range. (A color version of this figure is available in the online journal.) 


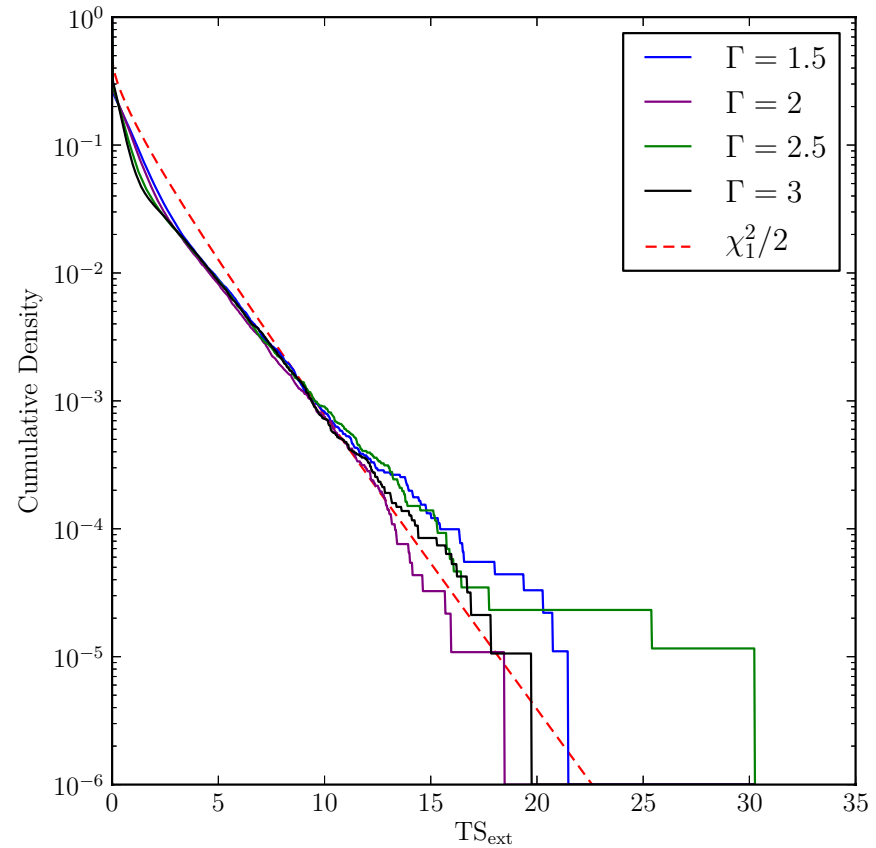

Figure 5. Cumulative distribution of $\mathrm{TS}_{\mathrm{ext}}$ for sources simulated on top of the Galactic diffuse and isotropic background.

(A color version of this figure is available in the online journal.)

background. Our simulation setup was the same as above except that the sources were simulated on top of and analyzed assuming the presence of the standard Galactic diffuse and isotropic background models used in 2FGL. In our simulations, we selected our sources to have random positions on the sky such that they were within $5^{\circ}$ of the Galactic plane. This probes the brightest and most strongly contrasting areas of the Galactic background.

To limit the number of tests, we selected only one flux level for each of the four spectral indices and we performed this test only in the 1-100 GeV energy range. As described below, the fluxes were selected so that TS $\sim 50$. We do not expect to be able to spatially resolve sources at lower fluxes than these, and the results for much brighter sources are less likely to be affected by the structured background.

Because the Galactic diffuse emission is highly structured with strong gradients, the point-source sensitivity can vary significantly across the Galactic plane. To account for this, we scaled the flux (for a given spectral index) so that the source always has approximately the same signal-to-noise ratio:

$$
F(\boldsymbol{x})=F(\mathrm{GC}) \times\left(\frac{B(\boldsymbol{x})}{B(\mathrm{GC})}\right)^{1 / 2} .
$$

Here, $\boldsymbol{x}$ is the position of the simulated source, $F$ is the integral flux of the source from $100 \mathrm{MeV}$ to $100 \mathrm{GeV}, F(\mathrm{GC})$ is the same quantity if the source was at the Galactic center, $B$ is the integral of the Galactic diffuse and isotropic emission from $1 \mathrm{GeV}$ to $100 \mathrm{GeV}$ at the position of the source, and $B(\mathrm{GC})$ is the same quantity if the source was at the Galactic center. For the four spectral models, Table 1 lists $F(\mathrm{GC})$ and the average value of TS.

For each spectrum, we performed $\sim 90,000$ simulations. Figure 5 shows the cumulative density of $\mathrm{TS}_{\mathrm{ext}}$ for each spectrum. For small values of $\mathrm{TS}_{\mathrm{ext}}$, there is good agreement between the simulations and theory. For the highest values of
$\mathrm{TS}_{\text {ext }}$, there is possibly a small discrepancy, but the discrepancy is not statistically significant. Therefore, we are confident we can use $\mathrm{TS}_{\text {ext }}$ as a robust measure of statistical significance when testing LAT-detected sources for extension.

\subsection{Extended Source Simulations over a Structured Background}

We also performed a Monte Carlo study to show that incorrectly modeling the spatial extension of an extended source does not substantially bias the spectral fit of the source, although it does alter the value of the TS. To assess this, we simulated the spatially extended ring-type SNR W44. We selected W44 because it is the most significant extended source detected by the LAT that has a non-radially symmetric photon distribution (Abdo et al. 2010h).

W44 was simulated with a power-law spectral model with an integral flux of $7.12 \times 10^{-8} \mathrm{ph} \mathrm{cm}^{-2} \mathrm{~s}^{-1}$ in the energy range from $1 \mathrm{GeV}$ to $100 \mathrm{GeV}$ and a spectral index of 2.66 (see Section 7).

W44 was simulated with the elliptical ring spatial model described in Abdo et al. (2010h). For reference, the ellipse has a semi-major axis of 0.3 , a semi-minor axis of 0.19 , a position angle of $147^{\circ}$ measured east of celestial north, and the ring's inner radius is $75 \%$ of the outer radius.

We used a simulation setup similar to that described in Section 3.2, but the simulations were over the two-year interval of the 2FGL catalog. In the simulations, we did not include the finite energy resolution of the LAT to isolate any effects due to changing the assumed spatial model. The fitting code we use also ignores this energy dispersion and the potential bias introduced by this will be discussed in an upcoming paper by the LAT Collaboration (Abdo et al. 2012a). In total, we performed 985 independent simulations.

The simulated sources were fit using a point-like spatial model, a radially symmetric Gaussian spatial model, a uniform disk spatial model, an elliptical disk spatial model, and finally with an elliptical ring spatial model. We obtained the best-fit spatial parameters using pointlike and, with these parameters, obtained the best-fit spectral parameters using gtlike.

Figure 6(a) shows that the significance of W44 in the simulations is very large (TS $\sim 3500$ ) for a model with a point-like source hypothesis. Figure 6(b) shows that the significance of the spatial extension is also large $\left(\mathrm{TS}_{\text {ext }} \sim 250\right)$. On average $\mathrm{TS}_{\mathrm{ext}}$ is somewhat larger when fitting the sources with more accurate spatial models. This shows that assuming an incorrect spatial model will cause the source's significance to be underestimated. Figure 6(c) shows that the sources were fit better when assuming an elliptical disk spatial model compared to a uniform disk spatial model $\left(\mathrm{TS}_{\text {elliptical disk }}-\mathrm{TS}_{\text {disk }} \sim 30\right.$ ). Finally, Figure 6(d) shows that the sources were fit somewhat better assuming an elliptical ring spatial model compared to an elliptical disk spatial model $\left(\mathrm{TS}_{\text {elliptical ring }}-\mathrm{TS}_{\text {elliptical disk }} \sim 9\right.$ ). This shows that the LAT has some additional power to resolve substructure in bright extended sources.

Figures 7(a) and (b) clearly show that no significant bias is introduced by modeling the source as extended but with an inaccurate spatial model, while a point-like source modeling results in a $\sim 10 \%$ and $\sim 0.125$ bias in the fit flux and index, respectively. Furthermore, Figure 7(c) shows that the $r_{68}$ estimate of the extension size is very mildly biased $(\sim 10 \%)$ toward higher values when inaccurate spatial models are used, and thus represents a reasonable measurement of the true $68 \%$ containment radius for the source. For the elliptical spatial models, $r_{68}$ is computed by numeric integration. 

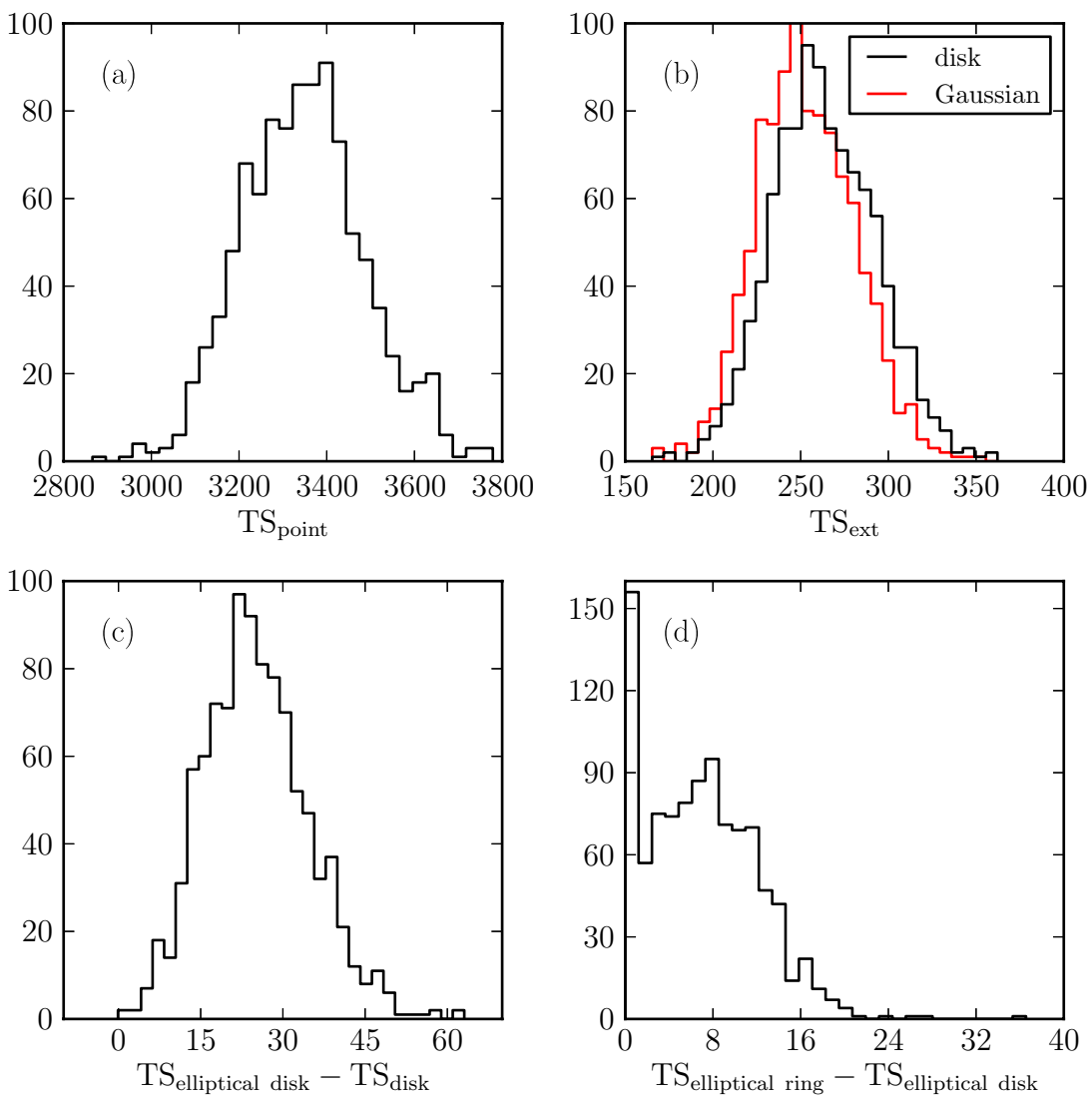

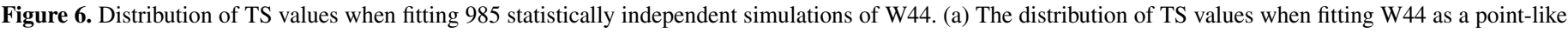

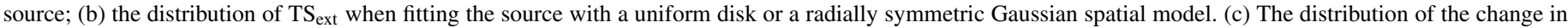

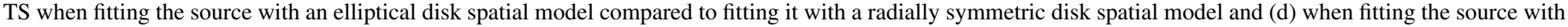
an elliptical ring spatial model compared to an elliptical disk spatial model.

(A color version of this figure is available in the online journal.)

\section{EXTENDED SOURCE DETECTION THRESHOLD}

We calculated the LAT flux threshold to detect spatial extent. We define the detection threshold as the flux at which the value of $\mathrm{TS}_{\text {ext }}$ averaged over many statistical realizations is $\left\langle\mathrm{TS}_{\text {ext }}\right\rangle=16$ (corresponding to a formal $4 \sigma$ significance) for a source of a given extension.

We used a simulation setup similar to that described in Section 3.1, but instead of point-like sources we simulated extended sources with radially symmetric uniform disk spatial models. Additionally, we simulated our sources over the twoyear time range included in the 2FGL catalog. For each extension and spectral index, we selected a flux range which bracketed $\mathrm{TS}_{\text {ext }}=16$ and performed an extension test for $>100$ independent realizations of 10 fluxes in the range. We calculated $\left\langle\mathrm{TS}_{\text {ext }}\right\rangle=16$ by fitting a line to the flux and $\mathrm{TS}_{\mathrm{ext}}$ values in the narrow range.

Figure 8 shows the threshold for sources of four spectral indices from 1.5 to 3 and extensions varying from $\sigma=0.1$ to 2.0 . The threshold is high for small extensions when the source is small compared to the size of the PSF. It drops quickly with increasing source size and reaches a minimum around 0.5 . The threshold increases for large extended sources because the source becomes increasingly diluted by the background. Figure 8 shows the threshold using photons with energies between $100 \mathrm{MeV}$ and $100 \mathrm{GeV}$ and also using only photons with energies between $1 \mathrm{GeV}$ and $100 \mathrm{GeV}$. Except for very large or very soft sources, the threshold is not substantially improved by including photons with energies between $100 \mathrm{MeV}$ and $1 \mathrm{GeV}$. This is also demonstrated in Figure 1 which shows $\mathrm{TS}_{\text {ext }}$ for the SNR IC 443 computed independently in 12 energy bins between $100 \mathrm{MeV}$ and $100 \mathrm{GeV}$. For IC 443, which has a spectral index $\sim 2.4$ and an extension $\sim 0.35$, almost the entire increase in likelihood from optimizing the source extent in the model comes from energies above $1 \mathrm{GeV}$. Furthermore, other systematic errors become increasingly large at low energy. For our extension search (Section 9), we therefore used only photons with energies above $1 \mathrm{GeV}$.

Figure 9 shows the flux threshold as a function of source extension for different background levels $(1 \times, 10 \times$, and $100 \times$ the nominal background), different spectral indices, and two different energy ranges (1-100 GeV and 10-100 GeV). The detection threshold is higher for sources in regions of higher background. When studying sources only at energies above $1 \mathrm{GeV}$, the LAT detection threshold (defined as the $1-100 \mathrm{GeV}$ flux at which $\left\langle\mathrm{TS}_{\mathrm{ext}}\right\rangle=16$ ) depends less strongly on the spectral index of the source. The index dependence of the detection threshold is even weaker when considering only photons with energies above $10 \mathrm{GeV}$ because the PSF changes little from $10 \mathrm{GeV}$ to $100 \mathrm{GeV}$. Overlaid on Figure 9 are the LAT-detected extended sources that will be discussed in Sections 7 and 10 . The extension thresholds are tabulated in Table 2.

Finally, Figure 10 shows the projected detection threshold of the LAT to extension with a 10 year exposure against 10 times the isotropic background measured by EGRET. This background is representative of the background near the Galactic 

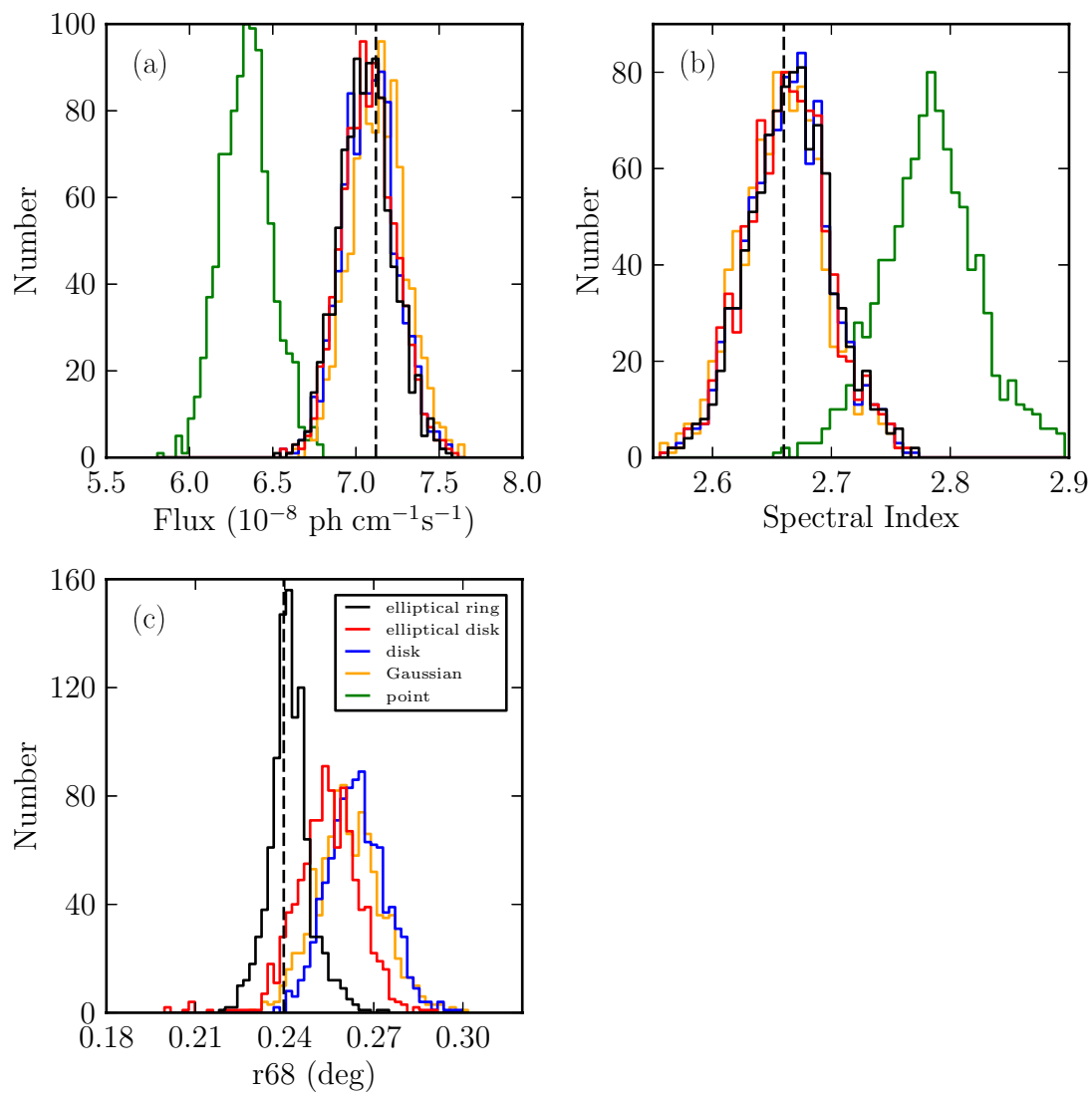

Figure 7. Distribution of fit parameters for the Monte Carlo simulations of W44. The plots show the distribution of best fit (a) flux, (b) spectral index, and (c) 68\% containment radius. The dashed vertical lines represent the simulated values of the parameters.

(A color version of this figure is available in the online journal.)

plane. For small extended sources, the threshold improves by a factor larger than the square root of the relative exposures because the LAT is signal limited at high energies where the present analysis is most sensitive. For large extended sources, the relevant background is over a larger spatial range and so the improvement is closer to a factor corresponding to the square root of the relative exposures that is caused by Poisson fluctuations in the background.

\section{TESTING AGAINST SOURCE CONFUSION}

It is impossible to discriminate using only LAT data between a spatially extended source and multiple point-like sources separated by angular distances comparable to or smaller than the size of the LAT PSF. To assess the plausibility of source confusion for sources with $\mathrm{TS}_{\mathrm{ext}} \geqslant 16$, we developed an algorithm to test if a region contains two point-like sources. The algorithm works by simultaneously fitting in pointlike the positions and spectra of the two point-like sources. To help with convergence, it begins by dividing the source into two spatially coincident point-like sources and then fitting the sum and difference of the positions of the two sources without any limitations on the fit parameters.

After simultaneously fitting the two positions and two spectra, we define $\mathrm{TS}_{2 \text { pts }}$ as twice the increase in the $\log$ of the likelihood fitting the region with two point-like sources compared to fitting the region with one point-like source:

$$
\mathrm{TS}_{2 \mathrm{pts}}=2 \log \left(\mathcal{L}_{2 \mathrm{pts}} / \mathcal{L}_{\mathrm{ps}}\right) .
$$

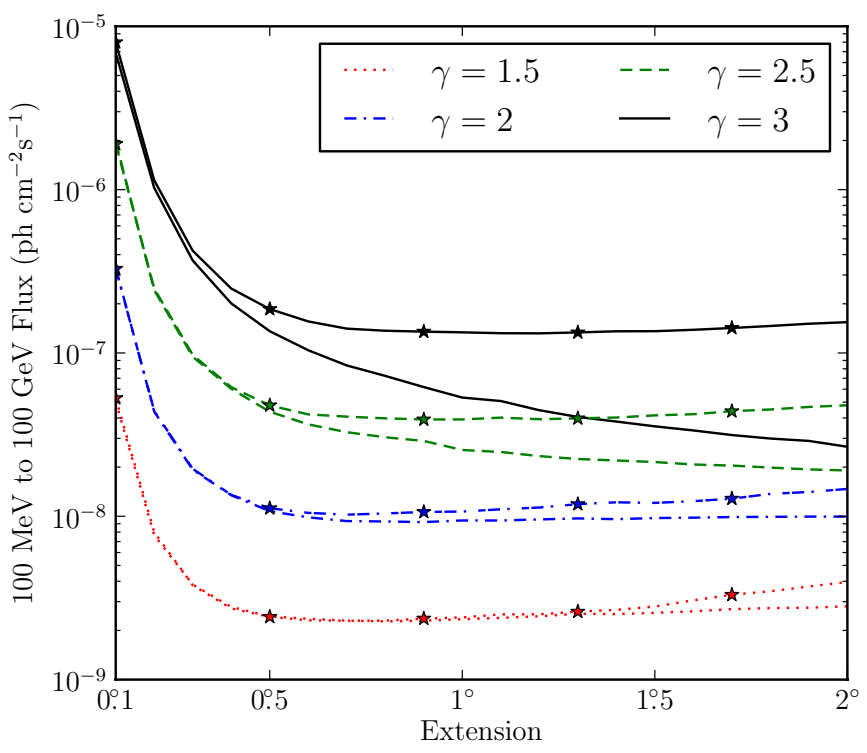

Figure 8. Detection threshold to resolve an extended source with a uniform disk model for a two-year exposure. All sources have an assumed power-law spectrum and the different line styles (colors in the online version) correspond to different simulated spectral indices. The lines with no markers correspond to the detection threshold using photons with energies between $100 \mathrm{MeV}$ and $100 \mathrm{GeV}$, while the lines with star-shaped markers correspond to the threshold using photons with energies between $1 \mathrm{GeV}$ and $100 \mathrm{GeV}$.

(A color version of this figure is available in the online journal.) 


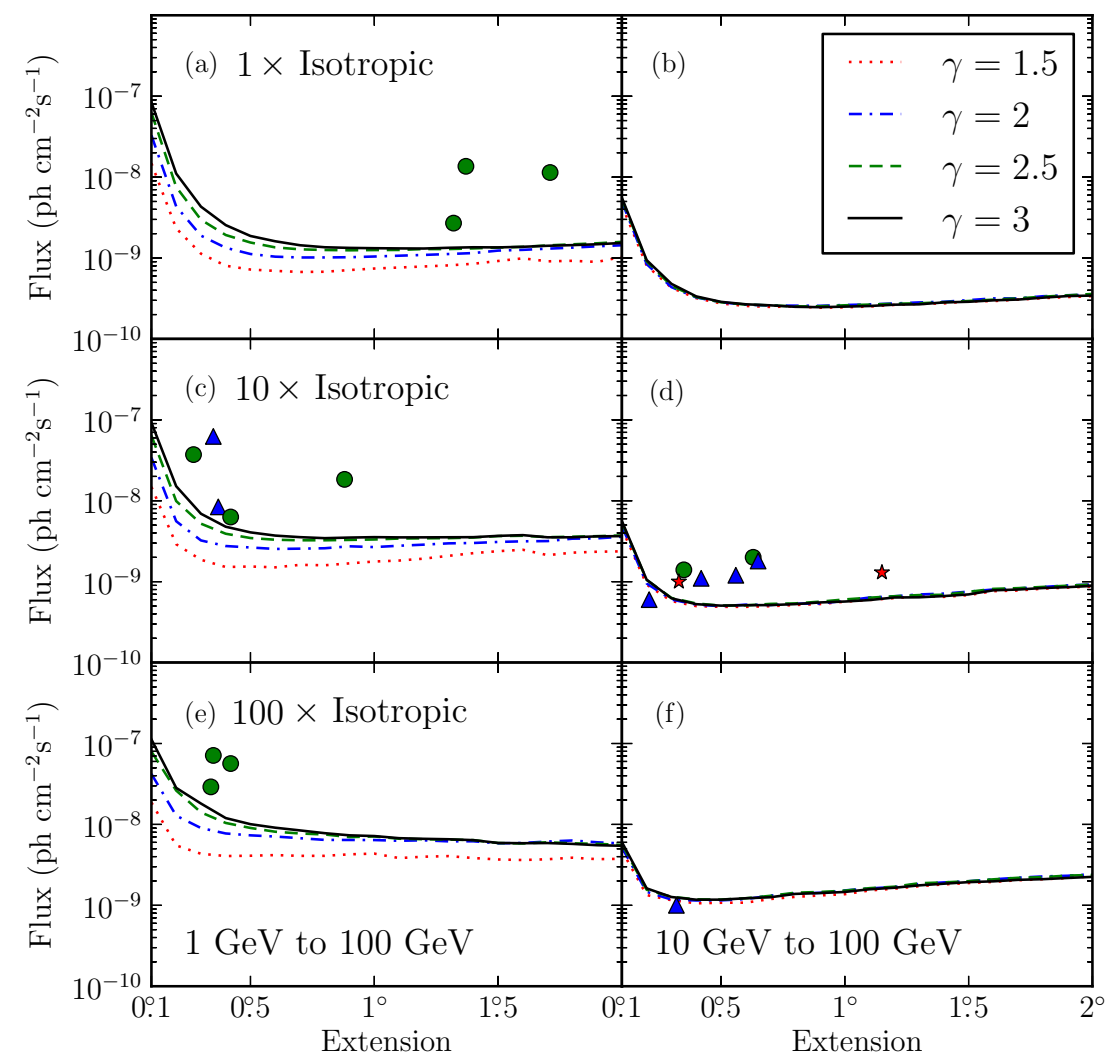

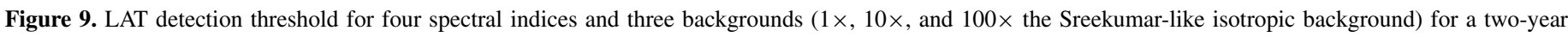

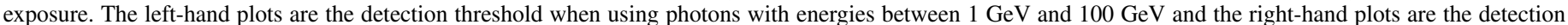

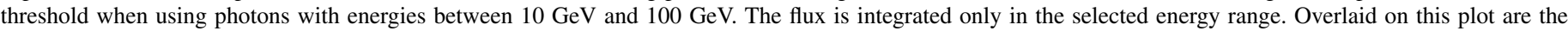

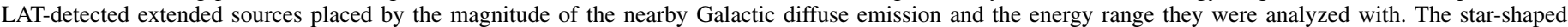

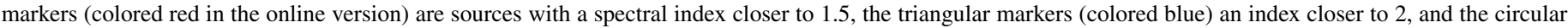
markers (colored green) an index closer to 2.5. The triangular marker in plot (d) below the sensitivity line is MSH $15-52$.

(A color version of this figure is available in the online journal.)

For the following analysis of LAT data, $\mathrm{TS}_{2 \mathrm{pts}}$ was computed by fitting the spectra of the two point-like sources in gtlike using the best-fit positions of the sources found by pointlike.

$\mathrm{TS}_{2 \mathrm{pts}}$ cannot be quantitatively compared to $\mathrm{TS}_{\text {ext }}$ using a simple likelihood-ratio test to evaluate which model is significantly better because the models are not nested (Protassov et al. 2002). Even though the comparison of $\mathrm{TS}_{\text {ext }}$ with $\mathrm{TS}_{2 \mathrm{pts}}$ is not a calibrated test, $\mathrm{TS}_{\mathrm{ext}}>\mathrm{TS}_{2 \mathrm{pts}}$ indicates that the likelihood for the extended source hypothesis is higher than for two pointlike sources and we only consider a source to be extended if $\mathrm{TS}_{\text {ext }}>\mathrm{TS}_{2 \mathrm{pts}}$.

We considered using the Bayesian information criterion (Schwarz 1978) as an alternative Bayesian formulation for this test, but it is difficult to apply to LAT data because it contains a term including the number of data points. For studying $\gamma$-ray sources in LAT data, we analyze relatively large regions of the sky to better define the contributions from diffuse backgrounds and nearby point sources. This is important for accurately evaluating source locations and fluxes but the fraction of data directly relevant to the evaluation of the parameters for the source of interest is relatively small.

As an alternative, we considered the Akaike information criterion (AIC) test (Akaike 1974). The AIC is defined as $\mathrm{AIC}=2 k-2 \log \mathcal{L}$, where $k$ is the number of parameters in the model. In this formulation, the best hypothesis is considered to be the one that minimizes the AIC. The first term penalizes models with additional parameters.
The two point-like sources hypothesis has three more parameters than the single extended source hypothesis (two more spatial parameters and two more spectral parameters compared to one extension parameter), so the comparison $\mathrm{AIC}_{\mathrm{ext}}<\mathrm{AIC}_{2 \mathrm{pts}}$ is formally equivalent to $\mathrm{TS}_{\mathrm{ext}}+6>\mathrm{TS}_{2 \mathrm{pts}}$. Our criterion for accepting extension $\left(\mathrm{TS}_{\mathrm{ext}}>\mathrm{TS}_{2 \mathrm{pts}}\right.$ ) is thus equivalent to requesting that the AIC-based empirical support for the two point-like sources model be "considerably less" than for the extended source model, following the classification by Burnham \& Anderson (2002).

We assessed the power of the $\mathrm{TS}_{\text {ext }}>\mathrm{TS}_{2 \text { pts }}$ test with a Monte Carlo study. We simulated one spatially extended source and fit it as both an extended source and as two point-like sources using pointlike. We then simulated two point-like sources and fit them with the same two hypotheses. By comparing the distribution of $\mathrm{TS}_{2 p t s}$ and $\mathrm{TS}_{\mathrm{ext}}$ computed by pointlike for the two cases, we evaluated how effective the $\mathrm{TS}_{\mathrm{ext}}>\mathrm{TS}_{2 \mathrm{pts}}$ test is at rejecting cases of source confusion as well as how likely it is to incorrectly reject that an extended source is spatially extended. All sources were simulated using the same time range as in Section 4 against a background 10 times the isotropic background measured by EGRET, representative of the background near the Galactic plane.

We did this study first in the energy range from $1 \mathrm{GeV}$ to $100 \mathrm{GeV}$ by simulating extended sources of flux $4 \times 10^{-9}$ $\mathrm{ph} \mathrm{cm}^{-2} \mathrm{~s}^{-1}$ integrated from $1 \mathrm{GeV}$ to $100 \mathrm{GeV}$ and a powerlaw spectral model with spectral index 2 . This spectrum was 
Table 2

Extension Detection Threshold

\begin{tabular}{|c|c|c|c|c|c|c|c|c|c|c|c|c|c|c|c|c|c|c|c|c|c|}
\hline$\gamma$ & BG & 0.1 & 0.2 & 0.3 & 0.4 & 0.5 & 0.6 & 0.7 & 0.8 & 0.9 & 1.0 & 1.1 & 1.2 & 1.3 & 1.4 & 1.5 & 1.6 & 1.7 & 1.8 & 1.9 & 2.0 \\
\hline \multicolumn{22}{|c|}{$E>1 \mathrm{GeV}$} \\
\hline \multirow[t]{3}{*}{1.5} & $1 \times$ & 148.1 & 23.3 & 11.3 & 8.0 & 7.2 & 6.9 & 6.7 & 6.8 & 7.1 & 7.4 & 7.6 & 7.9 & 8.1 & 8.5 & 9.2 & 9.9 & 9.1 & 9.2 & 9.0 & 10.3 \\
\hline & $10 \times$ & 148.4 & 29.0 & 18.7 & 15.2 & 15.4 & 15.0 & 16.1 & 16.0 & 16.8 & 17.7 & 18.2 & 19.3 & 20.9 & 22.5 & 23.8 & 24.8 & 21.3 & 22.8 & 23.4 & 23.7 \\
\hline & $100 \times$ & 186.8 & 55.0 & 43.4 & 40.7 & 41.0 & 41.8 & 40.9 & 40.9 & 42.7 & 43.6 & 38.4 & 39.9 & 40.6 & 38.4 & 36.9 & 36.3 & 37.1 & 38.8 & 37.2 & 37.6 \\
\hline \multirow[t]{3}{*}{2} & $1 \times$ & 328.4 & 43.4 & 18.9 & 13.4 & 11.2 & 10.4 & 10.2 & 10.2 & 10.2 & 10.4 & 10.7 & 10.9 & 11.2 & 11.5 & 12.4 & 12.6 & 13.0 & 13.4 & 14.0 & 14.4 \\
\hline & $10 \times$ & 341.0 & 55.9 & 32.3 & 27.6 & 26.5 & 25.4 & 25.6 & 25.9 & 27.4 & 26.8 & 27.8 & 28.7 & 29.8 & 30.1 & 31.0 & 31.5 & 31.7 & 34.0 & 34.3 & 35.9 \\
\hline & $100 \times$ & 420.5 & 128.3 & 90.2 & 77.3 & 73.3 & 70.8 & 67.5 & 64.3 & 64.2 & 64.1 & 62.8 & 63.6 & 61.7 & 61.9 & 58.4 & 59.0 & 61.4 & 63.3 & 60.1 & 58.1 \\
\hline \multirow[t]{3}{*}{2.5} & $1 \times$ & 627.1 & 5.6 & 29.8 & 19.3 & 15.5 & 13.5 & 12.8 & 12.6 & 12.5 & 12.5 & 12.6 & 12.9 & 12.9 & 13.1 & 13.5 & 13.7 & 14.3 & 14.8 & 15.2 & 15.8 \\
\hline & $10 \times$ & 638.9 & 99.1 & 52.1 & 39.1 & 34.6 & 33.0 & 32.5 & 32.5 & 32.8 & 33.2 & 34.1 & 34.3 & 34.5 & 35.1 & 36.6 & 36.9 & 35.5 & 36.0 & 36.5 & 37.3 \\
\hline & $100 x$ & 795.0 & 262.1 & 140.9 & 104.3 & 90.4 & 81.2 & 77.2 & 75.1 & 69.7 & 70.9 & 66.5 & 65.6 & 64.9 & 64.0 & 58.9 & 58.1 & 60.2 & 5 & 5 & 55.8 \\
\hline \multirow[t]{3}{*}{3} & $1 \times$ & 841.5 & 110.6 & & 25 & 18.7 & 16.1 & 14.4 & 13.6 & 13. & 13.2 & 13.1 & 13 & 13.4 & & 13.5 & 1 & 2 & 4 & 1 & 15.4 \\
\hline & $10 \times$ & 921.6 & 151.3 & 69.1 & 47.8 & 40.7 & 37.1 & 35.5 & 34.5 & 35. & 35.5 & 35.3 & 35.3 & 35.4 & 35 & 36.8 & 37.6 & 35.3 & 35.4 & 36.3 & 36.6 \\
\hline & $100 \times$ & 1124.1 & 282.9 & 181.1 & 119.8 & 100.7 & 91.1 & 84.3 & 77.9 & 73.3 & 71.8 & 67.6 & 66.4 & 65.5 & 63.9 & 59.0 & 58.6 & 58.8 & 57.5 & 55.4 & 54.4 \\
\hline \multicolumn{22}{|c|}{$E>10 \mathrm{GeV}$} \\
\hline \multirow[t]{3}{*}{1.5} & $1 \times$ & & & & & 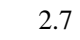 & 2 & & 2 & 2 & 2 & & & & & & & & & & 3.4 \\
\hline & $10 \times$ & 4 & & & & 4.9 & 4.9 & 5.0 & 5.2 & 5 & 5 & 3.9 & & & 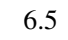 & 6.8 & 7.6 & 8 & & 5 & 8.7 \\
\hline & $100 \times$ & 4 & & & & 10.8 & 10.8 & 12.0 & 12.7 & 13 & 13 & 15 & 1 & 2 & 18.2 & 18.9 & 19 & 20.4 & 0 & 21.7 & 22.9 \\
\hline \multirow[t]{3}{*}{2} & $1 \times$ & 49.7 & & 4. & & 2. & 2 & 2 & 2.6 & 2 & 2 & 2 & & 2. & 2 & 3.0 & & & 3.4 & 3.5 & 3.5 \\
\hline & $10 \times$ & 48.6 & 9.5 & 6.0 & 5 & 5.0 & 5.2 & 5.2 & 5.3 & 5.4 & 5.8 & 6.4 & 6.6 & 7.0 & 7.1 & 7.5 & 8.0 & 8.3 & 8.6 & 9.0 & 9.2 \\
\hline & $100 \times$ & 51.8 & 14.7 & 11.8 & 11.5 & 11.5 & 11.9 & 13.2 & 14.0 & 14.3 & 15.3 & 16.2 & 16.9 & 18.4 & 19.2 & 19.8 & 21.0 & 22.0 & 22.8 & 23.2 & 24.3 \\
\hline \multirow[t]{3}{*}{2.5} & $1 \times$ & 53.1 & 9.1 & 4.5 & 3.3 & 2.8 & 2.7 & 2.6 & 2.5 & 2.5 & 2.6 & 2.7 & 2.7 & 2.8 & 2.8 & 2.9 & 3.1 & 3.2 & 3.3 & 3.5 & 3.6 \\
\hline & $10 \times$ & 53.7 & 10.5 & 6.3 & 5.4 & 5.1 & 5.1 & 5.3 & 5.4 & 5.7 & 6.0 & 6.3 & 6.6 & 6.8 & 6.9 & 7.5 & 8.1 & 8.3 & 8.6 & 8.9 & 9.2 \\
\hline & $100 \times$ & 57.0 & 15.6 & 12.7 & 11.9 & 11.8 & 12.2 & 13.1 & 14.3 & 14.6 & 15.2 & 16.3 & 17.0 & 18.8 & 19.2 & 19.9 & 21.0 & 21.9 & 22.3 & 23.3 & 23.7 \\
\hline \multirow[t]{3}{*}{3} & $1 \times$ & 55.5 & 9.4 & 4.8 & 3.4 & 2.9 & 2.7 & 2.6 & 2.5 & 2.5 & 2.5 & 2.6 & 2.7 & 2.7 & 2.8 & 2.9 & 3.0 & 3.1 & 3.2 & 3.4 & 3.4 \\
\hline & $10 \times$ & 56.0 & 10.5 & 6.2 & 5.3 & 5.1 & 5.1 & 5.1 & 5.3 & 5.5 & 5.7 & 5.9 & 6.4 & 6.4 & 6.6 & 7.0 & 7.8 & 8.0 & 8.3 & 8.6 & 8.9 \\
\hline & $100 \times$ & 60.3 & 16.2 & 12.7 & 11.7 & 11.8 & 12.2 & 12.6 & 13.8 & 14.2 & 14.6 & 15.8 & 16.5 & 17.6 & 18.5 & 19.4 & 19.8 & 20.7 & 21.0 & 21.8 & 22.5 \\
\hline
\end{tabular}

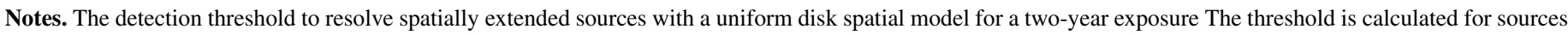

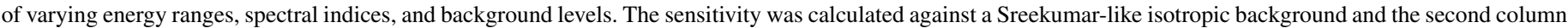

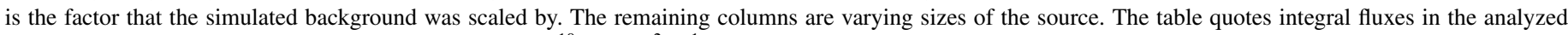
energy range (1-100 GeV or $10-100 \mathrm{GeV})$ in units of $10^{-10} \mathrm{ph} \mathrm{cm}^{-2} \mathrm{~s}^{-1}$.

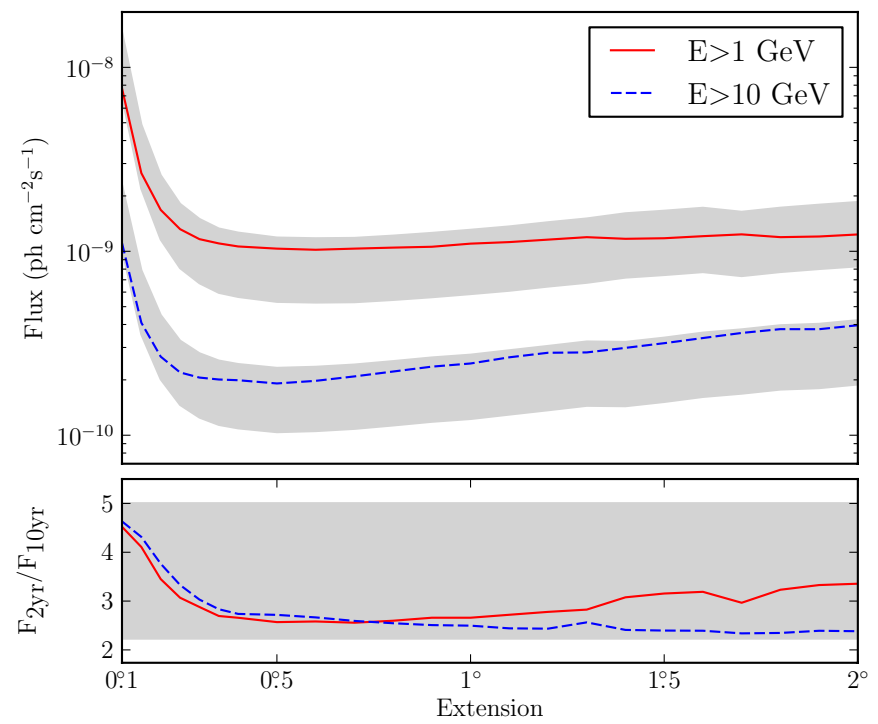

Figure 10. Projected detection threshold of the LAT to extension after 10 years for a power-law source of spectral index 2 against 10 times the isotropic background in the energy range from $1 \mathrm{GeV}$ to $100 \mathrm{GeV}$ (solid line colored red in the online version) and 10-100 GeV (dashed line colored blue). The shaded gray regions represent the detection threshold assuming the sensitivity improves from 2 to 10 years by the square root of the exposure (top edge) and linearly with exposure (bottom edge). The lower plot shows the factor increase in sensitivity. For small extended sources, the detection threshold of the LAT to the extension of a source will improve by a factor larger than the square root of the exposure.

(A color version of this figure is available in the online journal.) picked to be representative of the new extended sources that were discovered in the following analysis when looking in the $1-100 \mathrm{GeV}$ energy range (see Section 10 ). We simulated these sources using uniform disk spatial models with extensions varying up to $1^{\circ}$. Figure 11 (a) shows the distribution of $\mathrm{TS}_{\mathrm{ext}}$ and $\mathrm{TS}_{2 \mathrm{pts}}$ and Figure 11(c) shows the distribution of $\mathrm{TS}_{\mathrm{ext}}-\mathrm{TS}_{2 \mathrm{pts}}$ as a function of the simulated extension of the source for 200 statistically independent simulations.

Figure 12(a) shows the same plot but when fitting two simulated point-like sources each with half of the flux of the spatially extended source and with the same spectral index as the extended source. Finally, Figure 12(c) shows the same plot with each point-like source having the same flux but different spectral indices. One point-like source had a spectral index of 1.5 and the other an index of 2.5. These indices are representative of the range of indices of LAT-detected sources.

The same four plots are shown in Figures 11(b), (d), and 12(b), and (d) but this time when analyzing a source of flux $10^{-9} \mathrm{ph} \mathrm{cm}^{-2} \mathrm{~s}^{-1}$ (integrated from $10 \mathrm{GeV}$ to $100 \mathrm{GeV}$ ) only in the $10-100 \mathrm{GeV}$ energy range. This flux is typical of the new extended sources discovered using only photons with energies between $10 \mathrm{GeV}$ and $100 \mathrm{GeV}$ (see Section 10).

Several interesting conclusions can be made from this study. As one would expect, $\mathrm{TS}_{\mathrm{ext}}-\mathrm{TS}_{2 \mathrm{pts}}$ is mostly positive when fitting the simulated extended sources. In the 1-100 GeV analysis, only 11 of the 200 simulated extended sources had $\mathrm{TS}_{\text {ext }}>16$ but were incorrectly rejected due to $\mathrm{TS}_{2 \mathrm{pts}}$ being greater than $\mathrm{TS}_{\mathrm{ext}}$. In the $10-100 \mathrm{GeV}$ analysis, only 7 of the 200 sources were incorrectly rejected. From this, we conclude 


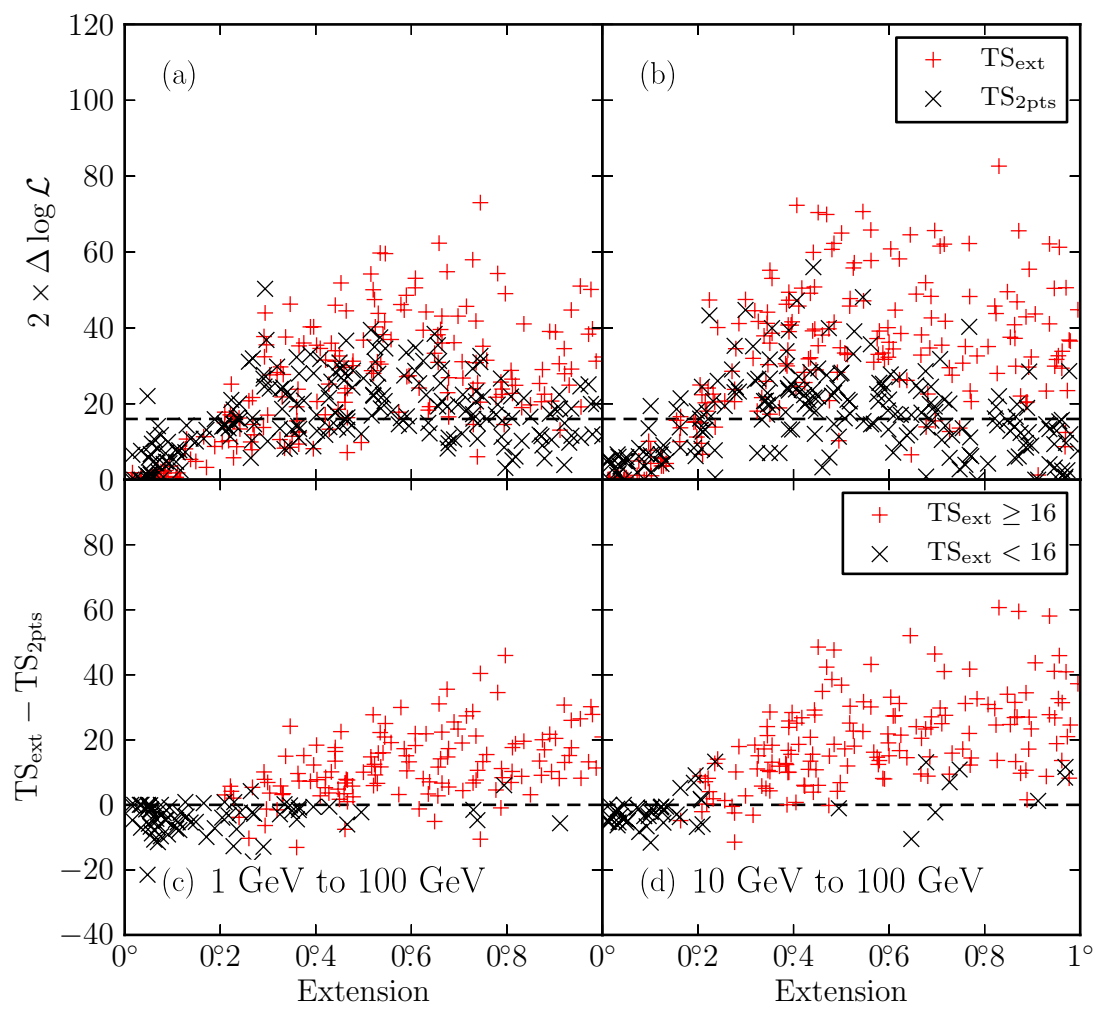

Figure 11. (a) and (b) are the distribution of $\mathrm{TS}_{\text {ext }}$ and of $\mathrm{TS}_{2 \mathrm{pts}}$ when fitting simulated spatially extended sources of varying sizes as both an extended source and as two point-like sources. (c) and (d) are the distribution of $\mathrm{TS}_{\text {ext }}-\mathrm{TS}_{2 \mathrm{pts}}$ for the same simulated sources. (a) and (c) represent sources fit in the 1-100 GeV energy range and (b) and (d) represent sources fit in the 10-100 GeV energy range. In (c) and (d), the plus-shaped markers (colored red in the online version) are fits where $\mathrm{TS}_{\mathrm{ext}} \geqslant 16$.

(A color version of this figure is available in the online journal.)

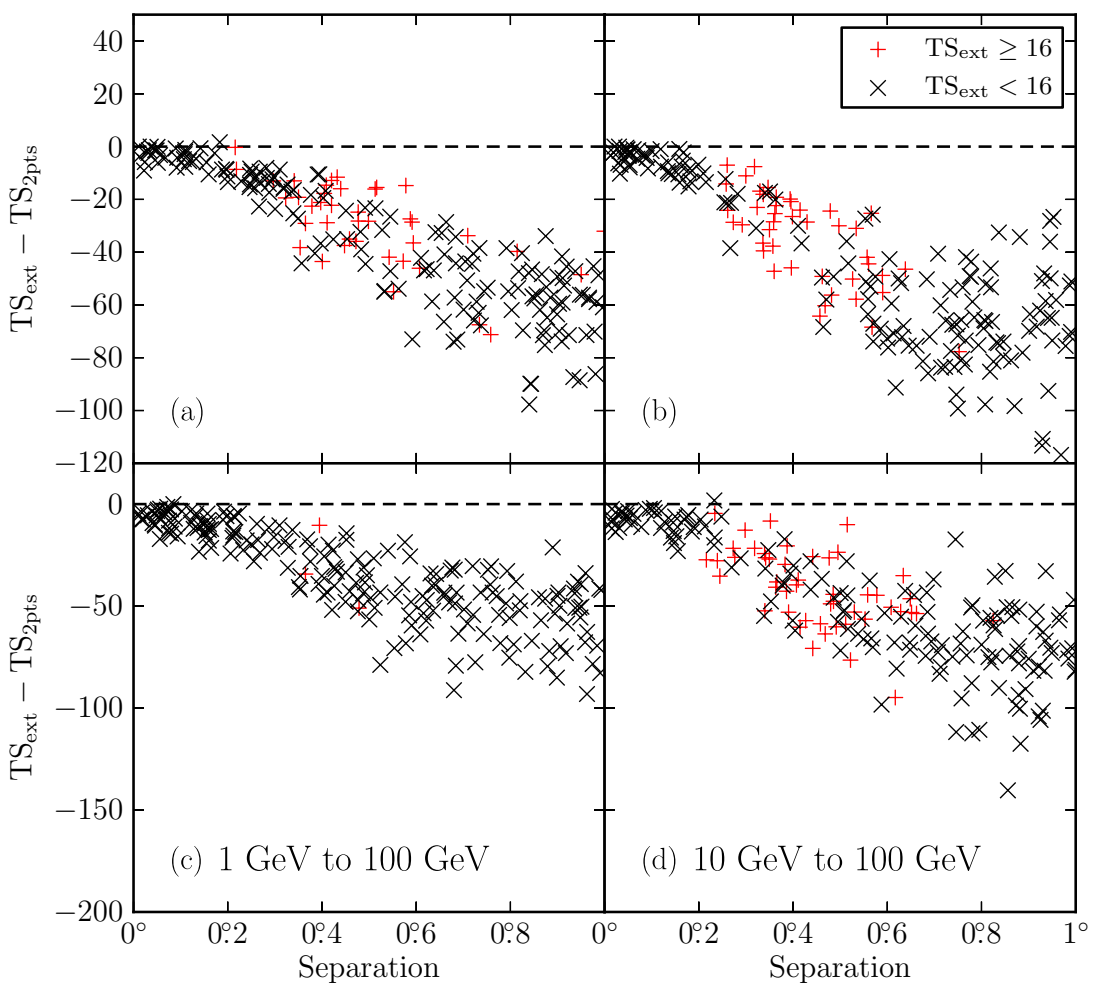

Figure 12. Distribution of $\mathrm{TS}_{\mathrm{ext}}-\mathrm{TS}_{2 \mathrm{pts}}$ when fitting two simulated point-like sources of varying separations as both an extended source and as two point-like sources. (a) and (b) represent simulations of two point-like sources with the same spectral index and (c) and (d) represent simulations of two point-like sources with different spectral indices. (a) and (c) fit the simulated sources in the 1-100 GeV energy range and (b) and (d) fit in the 10-100 GeV energy range. The plus-shaped markers (colored red in the online version) are fits where $\mathrm{TS}_{\mathrm{ext}} \geqslant 16$.

(A color version of this figure is available in the online journal.) 
that this test is unlikely to incorrectly reject truly spatially extended sources.

On the other hand, it is often the case that $\mathrm{TS}_{\text {ext }}>16$ when testing the two simulated point-like sources for extension. This is especially the case when the two sources had the same spectral index. Forty out of 200 sources in the $1-100 \mathrm{GeV}$ energy range and 43 out of 200 sources in the $10-100 \mathrm{GeV}$ energy range had $\mathrm{TS}_{\text {ext }}>16$. But in these cases, we always found the single extended source fit to be worse than the two point-like source fit. From this, we conclude that the $\mathrm{TS}_{\mathrm{ext}}>\mathrm{TS}_{2 \mathrm{pts}}$ test is powerful at discarding cases in which the true emission comes from two point-like sources.

The other interesting feature in Figures 11(a) and (b) is that for simulated extended sources with typical sizes $(\sigma \sim 0.5)$, one can often obtain almost as large an increase in likelihood fitting the source as two point-like sources $\left(\mathrm{TS}_{2 \mathrm{pts}} \sim \mathrm{TS}_{\mathrm{ext}}\right)$. This is because although the two point-like sources represent an incorrect spatial model, the second source has four additional degrees of freedom (two spatial and two spectral parameters) and can therefore easily model much of the extended source and statistical fluctuations in the data. This effect is most pronounced when using photons with energies between $1 \mathrm{GeV}$ and $100 \mathrm{GeV}$ where the PSF is broader.

From this Monte Carlo study, we can see the limits of an analysis with LAT data of spatially extended sources. Section 3.1 showed that we have a statistical test that finds when a LAT source is not well described by the PSF. But this test does not uniquely prove that the emission originates from spatially extended emission instead of from multiple unresolved sources. Demanding that $\mathrm{TS}_{\mathrm{ext}}>\mathrm{TS}_{2 \mathrm{pts}}$ is a powerful second test to avoid cases of simple confusion of two point-like sources. But it could always be the case that an extended source is actually the superposition of multiple point-like or extended sources that could be resolved with deeper observations of the region. There is nothing about this conclusion unique to analyzing LAT data, but the broad PSF of the LAT and the density of sources expected to be $\mathrm{GeV}$ emitters in the Galactic plane makes this issue more significant for analyses of LAT data. When possible, multiwavelength information should be used to help select the best model of the sky.

\section{TEST OF 2LAC SOURCES}

For all following analyses of LAT data, we used the same two-year data set that was used in the 2FGL catalog spanning from 2008 August 4 to 2010 August 1. We applied the same acceptance cuts and we used the same P7_V6 Source class event selection and IRFs (Abdo et al. 2012a). When analyzing sources in pointlike, we used a circular $10^{\circ}$ region of interest (ROI) centered on our source and eight energy bins per logarithmic decade in energy. When refitting the region in gtlike using the best-fit spatial and spectral models from pointlike, we used the "binned likelihood" mode of gtlike on a $14^{\circ} \times 14^{\circ} \mathrm{ROI}$ with a pixel size of 0.03 .

Unless explicitly mentioned, we used the same background model as 2FGL to represent the Galactic diffuse, isotropic, and Earth limb emission. To compensate for possible residuals in the diffuse emission model, the Galactic emission was scaled by a power law and the normalization of the isotropic component was left free. Unless explicitly mentioned, we used all 2FGL sources within $15^{\circ}$ of our source as our list of background sources and we refit the spectral parameters of all sources within $2^{\circ}$ of the source.

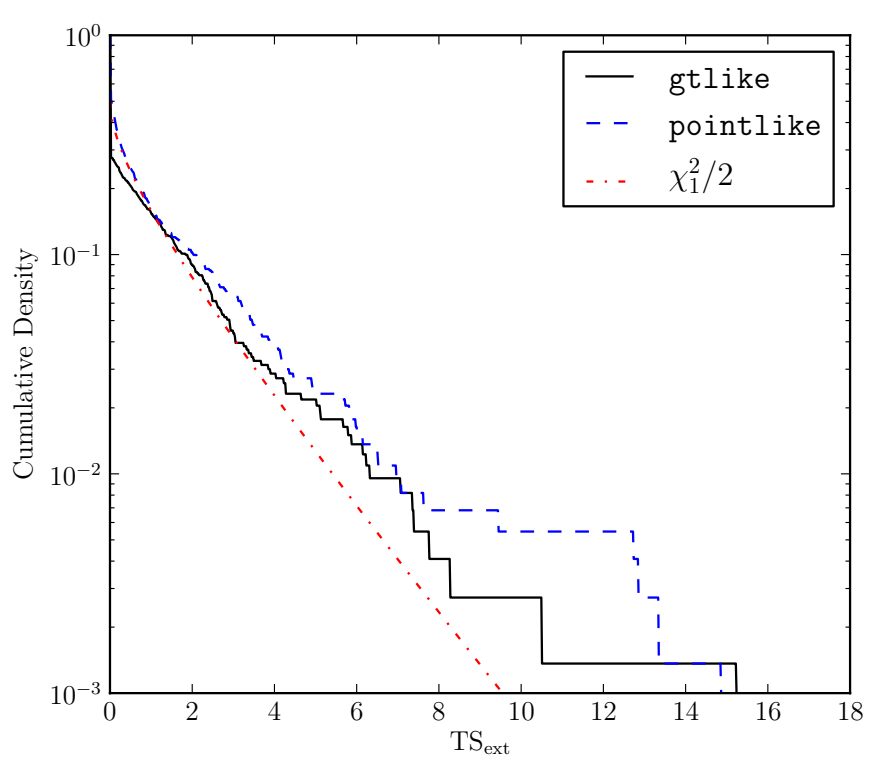

Figure 13. Cumulative density of $\mathrm{TS}_{\text {ext }}$ for the 733 clean AGNs in $2 \mathrm{LAC}$ that were significant above $1 \mathrm{GeV}$ calculated with pointlike (dashed line colored blue in the online version) and with gtlike (solid line colored black). AGNs are too far and too small to be resolved by the LAT. Therefore, the cumulative density of $\mathrm{TS}_{\mathrm{ext}}$ is expected to follow a $\chi_{1}^{2} / 2$ distribution (Equation (11), the dash-dotted line colored red).

(A color version of this figure is available in the online journal.)

To validate our method, we tested LAT sources associated with AGNs for extension. GeV emission from AGNs is believed to originate from collimated jets. Therefore AGNs are not expected to be spatially resolvable by the LAT and provide a good calibration source to demonstrate that our extension detection method does not misidentify point-like sources as being extended. We note that megaparsec-scale $\gamma$-ray halos around AGNs have been hypothesized to be resolvable by the LAT (Aharonian et al. 1994). However, no such halo has been discovered in the LAT data so far (Neronov et al. 2011).

Following 2FGL, the LAT Collaboration published the Second LAT AGN Catalog (2LAC), a list of high-latitude $\left(|b|>10^{\circ}\right)$ sources that had a high probability association with AGNs (Ackermann et al. 2011). 2LAC associated 1016 2FGL sources with AGNs. To avoid systematic problems with AGN classification, we selected only the 885 AGNs which made it into the clean AGN sub-sample defined in the 2LAC paper. An AGN association is considered clean only if it has a high probability of association $P \geqslant 80 \%$, if it is the only AGN associated with the 2FGL source, and if no analysis flags have been set for the source in the 2 FGL catalog. These last two conditions are important for our analysis. Source confusion may look like a spatially extended source and flagged 2FGL sources may correlate with unmodeled structure in the diffuse emission.

Of the 885 clean AGNs, we selected the 733 of these 2FGL sources which were significantly detected above $1 \mathrm{GeV}$ and fit each of them for extension. The cumulative density of $\mathrm{TS}_{\mathrm{ext}}$ for these AGNs is compared to the $\chi_{1}^{2} / 2$ distribution of Equation (11) in Figure 13. The $\mathrm{TS}_{\mathrm{ext}}$ distribution for the AGN shows reasonable agreement with the theoretical distribution and no AGN was found to be significantly extended $\left(\mathrm{TS}_{\text {ext }}>16\right)$. The observed discrepancy from the theoretical distribution is likely due to small systematics in our model of the LAT PSF and the Galactic diffuse emission (see Section 8). The discrepancy could also in a few cases be due to confusion with a nearby undetected source. We note that the Monte Carlo study 
Table 3

Analysis of the 12 Extended Sources Included in the 2FGL Catalog

\begin{tabular}{|c|c|c|c|c|c|c|c|c|}
\hline Name & $\begin{array}{c}\text { GLON } \\
\text { (deg) }\end{array}$ & $\begin{array}{l}\text { GLAT } \\
\text { (deg) }\end{array}$ & $\begin{array}{c}\sigma \\
(\operatorname{deg})\end{array}$ & $\mathrm{TS}$ & $\mathrm{TS}_{\mathrm{ext}}$ & $\begin{array}{c}\text { Pos Err } \\
\text { (deg) }\end{array}$ & Flux $^{a}$ & Index \\
\hline \multicolumn{9}{|c|}{$E>1 \mathrm{GeV}$} \\
\hline SMC & 302.59 & -44.42 & $1.32 \pm 0.15 \pm 0.31$ & 95.0 & 52.9 & 0.14 & $2.7 \pm 0.3$ & $2.48 \pm 0.19$ \\
\hline LMC & 279.26 & -32.31 & $1.37 \pm 0.04 \pm 0.11$ & 1127.9 & 909.9 & 0.04 & $13.6 \pm 0.6$ & $2.43 \pm 0.06$ \\
\hline IC 443 & 189.05 & 3.04 & $0.35 \pm 0.01 \pm 0.04$ & 10692.9 & 554.4 & 0.01 & $62.4 \pm 1.1$ & $2.22 \pm 0.02$ \\
\hline Vela X & 263.34 & -3.11 & 0.88 & & & & & \\
\hline Centaurus A & 309.52 & 19.42 & $\sim 10$ & & & & & \\
\hline W28 & 6.50 & -0.27 & $0.42 \pm 0.02 \pm 0.05$ & 1330.8 & 163.8 & 0.01 & $56.5 \pm 1.8$ & $2.60 \pm 0.03$ \\
\hline W30 & 8.61 & -0.20 & $0.34 \pm 0.02 \pm 0.02$ & 464.8 & 76.0 & 0.02 & $29.1 \pm 1.5$ & $2.56 \pm 0.05$ \\
\hline W44 & 34.69 & -0.39 & $0.35 \pm 0.02 \pm 0.02$ & 1917.0 & 224.8 & 0.01 & $71.2 \pm 0.5$ & $2.66 \pm 0.00$ \\
\hline W51C & 49.12 & -0.45 & $0.27 \pm 0.02 \pm 0.04$ & 1823.4 & 118.9 & 0.01 & $37.2 \pm 1.3$ & $2.34 \pm 0.03$ \\
\hline Cygnus Loop & 74.21 & -8.48 & $1.71 \pm 0.05 \pm 0.06$ & 357.9 & 246.0 & 0.06 & $11.4 \pm 0.7$ & $2.50 \pm 0.10$ \\
\hline \multicolumn{9}{|c|}{$E>10 \mathrm{GeV}$} \\
\hline MSH $15-52^{b}$ & 320.39 & -1.22 & $0.21 \pm 0.04 \pm 0.04$ & 76.3 & 6.6 & 0.03 & $0.6 \pm 0.1$ & $2.20 \pm 0.22$ \\
\hline HESS J1825-137 ${ }^{\mathrm{b}}$ & 17.56 & -0.47 & $0.65 \pm 0.04 \pm 0.02$ & 59.7 & 33.8 & 0.05 & $1.6 \pm 0.2$ & $1.63 \pm 0.22$ \\
\hline
\end{tabular}

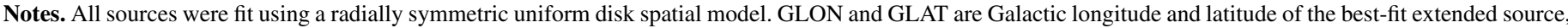

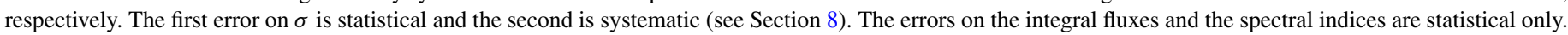
Pos Err is the error on the position of the source. Vela X and the Centaurus A Lobes were not fit in our analysis but are included for completeness.

${ }^{a}$ Integral flux in units of $10^{-9} \mathrm{ph} \mathrm{cm}^{-2} \mathrm{~s}^{-1}$ and integrated in the fit energy range (either $1-100 \mathrm{GeV}$ or $10-100 \mathrm{GeV}$ ).

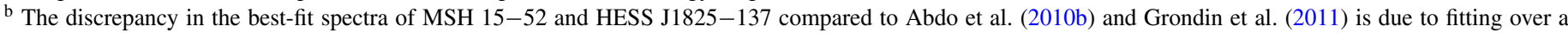
different energy range.

of Section 3.1 effectively used perfect IRFs and a perfect model of the sky. The overall agreement with the expected distribution demonstrates that we can use $\mathrm{TS}_{\mathrm{ext}}$ as a measure of the statistical significance of the detection of the extension of a source.

We note that the LAT PSF used in this study was determined empirically by fitting the distributions of gamma rays around bright AGNs (see Section 8). Finding that the AGNs we test are not extended is not surprising. This validation analysis is not suitable to reject any hypotheses about the existence of megaparsec-scale halos around AGNs.

\section{ANALYSIS OF EXTENDED SOURCES IDENTIFIED IN THE 2FGL CATALOG}

As further validation of our method for studying spatially extended sources, we reanalyzed the 12 spatially extended sources which were included in the 2FGL catalog (Nolan et al. 2012). Even though these sources had all been the subjects of dedicated analyses and separate publications, and had been fit with a variety of spatial models, it is valuable to show that these sources are significantly extended using our systematic method assuming radially symmetric uniform disk spatial models. On the other hand, for some of these sources a uniform disk spatial model does not well describe the observed extended emission and so the dedicated publications by the LAT Collaboration provide better models of these sources.

Six extended SNRs were included in the 2FGL catalog: W51C, IC 443, W28, W44, the Cygnus Loop, and W30 (Abdo et al. 2009a, 2010f, 2010h, 2010i, 2012b; Katagiri et al. 2011). Using photons with energies between $1 \mathrm{GeV}$ and $100 \mathrm{GeV}$, our analysis significantly detected that these six SNRs are spatially extended.

Two nearby satellite galaxies of the Milky Way the LMC and the SMC were included in the 2FGL catalog as spatially extended sources (Abdo et al. 2010c, 2010j). Their extensions were significantly detected using photons with energies between $1 \mathrm{GeV}$ and $100 \mathrm{GeV}$. Our fit extensions are comparable to the published result, but we note that the previous LAT Collabora- tion publication on the LMC used a more complicated two 2D Gaussian surface brightness profile when fitting it (Abdo et al. 2010j).

Three PWNe, MSH 15-52, Vela X, and HESS J1825-137, were fit as extended sources in the 2FGL analysis (Abdo et al. 2010b, 2010g; Grondin et al. 2011). In the present analysis, HESS J1825-137 was significantly detected using photons with energies between $10 \mathrm{GeV}$ and $100 \mathrm{GeV}$. To avoid confusion with the nearby bright pulsar PSR J1509-5850, MSH 15-52 had to be analyzed at high energies. Using photons with energies above $10 \mathrm{GeV}$, we fit the extension of MSH $15-52$ to be consistent with the published size but with $\mathrm{TS}_{\mathrm{ext}}=6.6$.

Our analysis was unable to resolve Vela $\mathrm{X}$ which would have required first removing the pulsed photons from the Vela pulsar which was beyond the scope of this paper. Our analysis also failed to detect a significant extension for the Centaurus A Lobes because the shape of the source is significantly different from a uniform radially symmetric disk (Abdo et al. 2010d).

Our analysis of these sources is summarized in Table 3. This table includes the best-fit positions and extensions of these sources when fitting them with a radially symmetric uniform disk model. It also includes the best-fit spectral parameters for each source. The positions and extensions of Vela $\mathrm{X}$ and the Centaurus A Lobes were taken from Abdo et al. (2010d, 2010g) and are included in this table for completeness.

\section{SYSTEMATIC ERRORS ON EXTENSION}

We developed two criteria for estimating systematic errors on the extensions of the sources. First, we estimated a systematic error due to uncertainty in our knowledge of the LAT PSF. Before launch, the LAT PSF was determined by detector simulations which were verified in accelerator beam tests (Atwood et al. 2009). However, in-flight data revealed a discrepancy above $3 \mathrm{GeV}$ in the PSF compared to the angular distribution of photons from bright AGNs (Abdo et al. 2012a). Subsequently, the PSF was fit empirically to bright AGNs and this empirical parameterization is used in the P7_V6 IRFs. To 
account for the uncertainty in our knowledge of the PSF, we refit our extended source candidates using the pre-flight Monte Carlo representation of the PSF and consider the difference in extension found using the two PSFs as a systematic error on the extension of a source. The same approach was used in Abdo et al. (2010i). We believe that our parameterization of the PSF from bright AGNs is substantially better than the Monte Carlo representation of the PSF so this systematic error is conservative.

We estimated a second systematic error on the extension of a source due to uncertainty in our model of the Galactic diffuse emission by using an alternative approach to modeling the diffuse emission which takes as input templates calculated by GALPROP ${ }^{15}$ but then fits each template locally in the surrounding region. The particular GALPROP model that was used as input is described in the analysis of the isotropic diffuse emission with LAT data (Abdo et al. 2010k). The intensities of various components of the Galactic diffuse emission were fitted individually using a spatial distribution predicted by the model. We considered separate contributions from cosmic-ray interactions with the molecular hydrogen, the atomic and ionized hydrogen, residual gas traced by dust (Grenier et al. 2005), and the interstellar radiation field. We further split the contributions from interactions with molecular and atomic hydrogen to the Galactic diffuse emission according to the distance from the Galactic center in which they are produced. Hence, we replaced the standard diffuse emission model by 18 individually fitted templates to describe individual components of the diffuse emission. A similar crosscheck was used in an analysis of RX J1713.7-3946 by the LAT Collaboration (Abdo et al. 2011).

It is not expected that this diffuse model is superior to the standard LAT model obtained through an all-sky fit. However, adding degrees of freedom to the background model can remove likely spurious sources that correlate with features in the Galactic diffuse emission. Therefore, this tests systematics that may be due to imperfect modeling of the diffuse emission in the region. Nevertheless, this alternative approach to modeling the diffuse emission does not test all systematics related to the diffuse emission model. In particular, because the alternative approach uses the same underlying gas maps, it is unable to be used to assess systematics due to insufficient resolution of the underlying maps. Structure in the diffuse emission that is not correlated with these maps will also not be assessed by this test.

We do not expect the systematic error due to uncertainties in the PSF to be correlated with the systematic error due to uncertainty in the Galactic diffuse emission. Therefore, the total systematic error on the extension of a source was obtained by adding the two errors in quadrature.

There is another systematic error on the size of a source due to issues of modeling nearby sources in crowded regions of the sky. It is beyond the scope of this paper to address this systematic error. Therefore, for sources in crowded regions the systematic errors quoted in this paper may not represent the full set of systematic errors associated with this analysis.

\section{EXTENDED SOURCE SEARCH METHOD}

Having demonstrated that we understand the statistical issues associated with analyzing spatially extended sources

\footnotetext{
15 GALPROP is a software package for calculating the Galactic $\gamma$-ray emission based on a model of cosmic-ray propagation in the Galaxy and maps of the distributions of the components of the interstellar medium (Strong \& Moskalenko 1998; Vladimirov et al. 2011). See also http://galprop.stanford.edu/ for details.
}

(Sections 3.1 and 6) and that our method can correctly analyze the extended sources included in 2FGL (Section 7), we applied this method to search for new spatially extended $\mathrm{GeV}$ sources. The data and general analysis setting are as described in Section 6.

Ideally, we would apply a completely blind and uniform search that tests the extension of each 2FGL source in the presence of all other 2FGL sources to find a complete list of all spatially extended sources. As our test of AGNs in Section 6 showed, at high Galactic latitude where the source density is not as large and the diffuse emission is less structured, this method works well.

But this is infeasible in the Galactic plane where we are most likely to discover new spatially extended sources. In the Galactic plane, this analysis is challenged by our imperfect model of the diffuse emission and by an imperfect model of nearby sources. The Monte Carlo study in Section 5 showed that the overall likelihood would greatly increase by fitting a spatially extended source as two point-like sources so we expect that spatially extended sources would be modeled in the 2FGL catalog as multiple point-like sources. Furthermore, the positions of other nearby sources in the region close to an extended source could be biased by not correctly modeling the extension of the source. The 2FGL catalog contains a list of sources significant at energies above $100 \mathrm{MeV}$ whereas we are most sensitive to spatial extension at higher energies. We therefore expect that at higher energies our analysis would be complicated by 2 FGL sources no longer significant and by 2FGL sources whose positions were biased by diffuse emission at lower energies.

To account for these issues, we first produced a large list of possibly extended sources employing very liberal search criteria and then refined the analysis of the promising candidates on a case-by-case basis. Our strategy was to test all point-like 2FGL sources for extension assuming they had a uniform radially symmetric disk spatial model and a power-law spectral model. Although not all extended sources are expected to have a shape very similar to a uniform disk, Section 2.4 showed that for many spatially extended sources the wide PSF of the LAT and limited statistics makes this a reasonable approximation. On the other hand, choosing this spatial model biases us against finding extended sources that are not well described by a uniform disk model such as shell-type SNRs.

Before testing for extension, we automatically removed from the background model all other 2FGL sources within 0.5 of the source. This distance is somewhat arbitrary, but was picked in hopes of finding extended sources with sizes on the order of $\sim 1^{\circ}$ or smaller. On the other hand, by removing these nearby background sources we expect to also incorrectly add to our list of extended source candidates point-like sources that are confused with nearby sources. To screen out obvious cases of source confusion, we performed the dual localization procedure described in Section 5 to compare the extended source hypothesis to the hypothesis of two independent pointlike sources.

As was shown in Section 4, little sensitivity is gained by using photons with energies below $1 \mathrm{GeV}$. In addition, the broad PSF at low energy makes the analysis more susceptible to systematic errors arising from source confusion due to nearby soft pointlike sources and by uncertainties in our modeling of the Galactic diffuse emission. For these reasons, we performed our search using only photons with energies between $1 \mathrm{GeV}$ and $100 \mathrm{GeV}$.

We also performed a second search for extended sources using only photons with energies between $10 \mathrm{GeV}$ and 

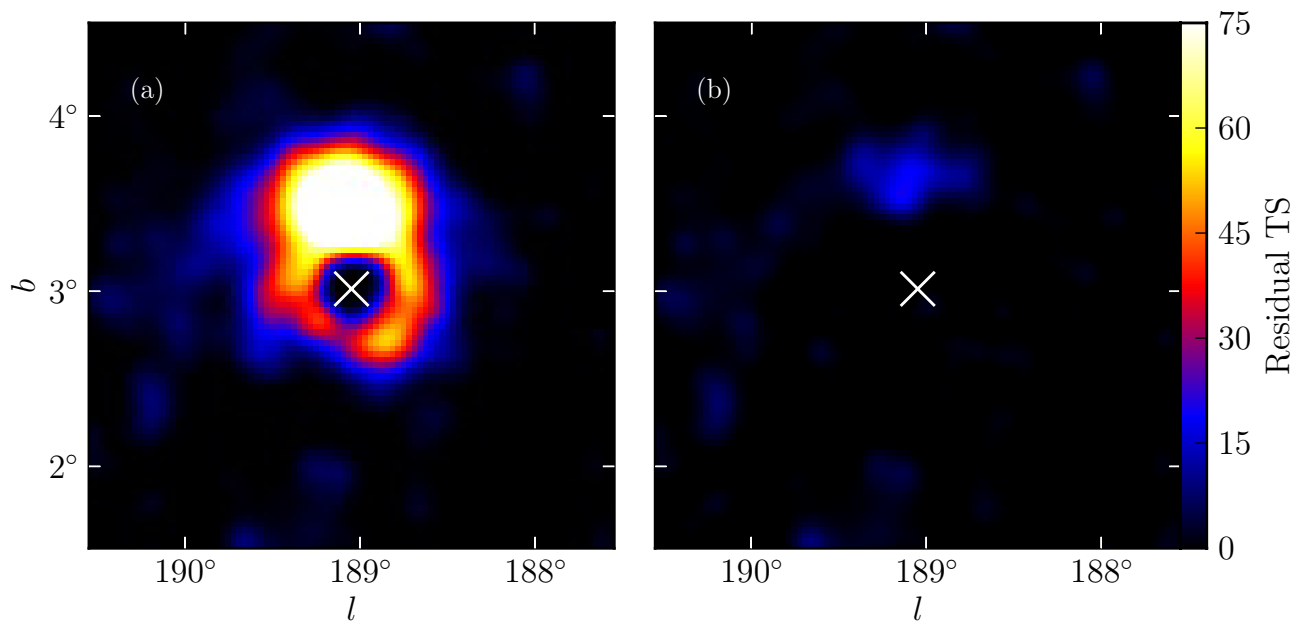

Figure 14. TS map generated for the region around the SNR IC 443 using photons with energies between $1 \mathrm{GeV}$ and $100 \mathrm{GeV}$. (a) TS map after subtracting IC 443 modeled as a point-like source. (b) Same as (a), but IC 443 modeled as an extended source. The cross represents the best-fit position of IC 443.

(A color version of this figure is available in the online journal.)

$100 \mathrm{GeV}$. Although this approach tests the same sources, it is complementary because the Galactic diffuse emission is even less dominant above $10 \mathrm{GeV}$ and because source confusion is less of an issue. A similar procedure was used to detect the spatial extensions of MSH 15-52 and HESS J1825-137 with the LAT (Abdo et al. 2010b; Grondin et al. 2011).

When we applied this test to the 1861 point-like sources in the 2FGL catalog, our search found 117 extended source candidates in the $1-100 \mathrm{GeV}$ energy range and 11 extended source candidates in the $10-100 \mathrm{GeV}$ energy range. Most of the extended sources found above $10 \mathrm{GeV}$ were also found above $1 \mathrm{GeV}$ and in many cases multiple nearby point-like sources were found to be extended even though they fit the same emission region. For example, the sources 2FGL J1630.2-4752, 2FGL J1632.4-4753c, 2FGL J1634.4-4743c, and 2FGL J1636.3-4740c were all found to be spatially extended in the $10-100 \mathrm{GeV}$ energy range even though they all fit to similar positions and sizes. For these situations, we manually discarded all but one of the 2FGL sources.

Similarly, many of these sources were confused with nearby point-like sources or influenced by large-scale residuals in the diffuse emission. To help determine which of these fits found truly extended sources and when the extension was influenced by source confusion and diffuse emission, we generated a series of diagnostic plots. For each candidate, we generated a map of the residual TS by adding a new point-like source of spectral index 2 into the region at each position and finding the increase in likelihood when fitting its flux. Figure 14 shows this map around the most significantly extended source IC 443 when it is modeled both as a point-like source and as an extended source. The residual TS map indicates that the spatially extended model for IC 443 is a significantly better description of the observed photons and that there is no TS $>25$ residual in the region after modeling the source as being spatially extended. We also generated plots of the sum of all counts within a given distance of the source and compared them to the model predictions assuming the emission originated from a point-like source. An example radial integral plot is shown for the extended source IC 443 in Figure 1. For each source, we also made diffuseemission-subtracted smoothed counts maps (shown for IC 443 in Figure 1).

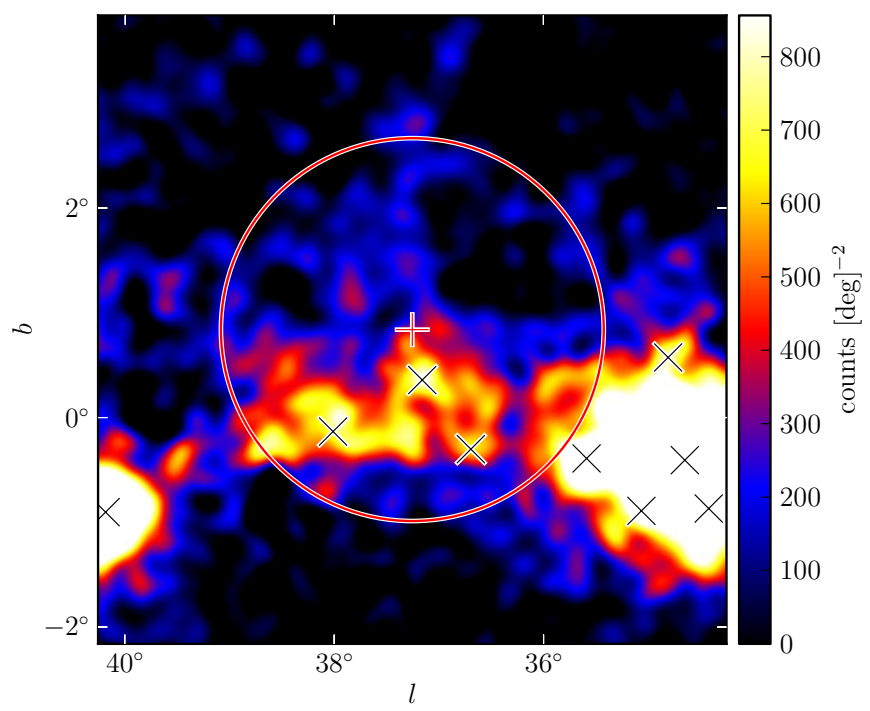

Figure 15. Diffuse-emission-subtracted 1-100 GeV counts map of the region around 2FGL J1856.2+0450c smoothed by a 0.12 D Gaussian kernel. The plusshaped marker and circle (colored red in the online version) represent the center and size of the source fit with a radially symmetric uniform disk spatial model. The black crosses represent the positions of other 2FGL sources. The extension is statistically significant, but the extension encompasses many 2FGL sources and the emission does not look to be uniform. Although the fit is statistically significant, it likely corresponds to residual features of inaccurately modeled diffuse emission picked up by the fit.

(A color version of this figure is available in the online journal.)

We found by visual inspection that in many cases our results were strongly influenced by large-scale residuals in the diffuse emission and hence the extension measure was unreliable. This was especially true in our analysis of sources in the $1-100 \mathrm{GeV}$ energy range. An example of such a case is 2FGL J1856.2+0450c analyzed in the $1-100 \mathrm{GeV}$ energy range. Figure 15 shows a diffuse-emission-subtracted smoothed counts map for this source with the best-fit extension of the source overlaid. There appear to be large-scale residuals in the diffuse emission in this region along the Galactic plane. As a result, 2FGL J1856.2+0450c is fit to an extension of $\sim 2^{\circ}$ and the result is statistically significant with $\mathrm{TS}_{\mathrm{ext}}=45.4$. However, by looking at the residuals it is clear that this complicated region is not well modeled. We manually discard sources like this. 
We only selected extended source candidates in regions that did not appear dominated by these issues and where there was a multiwavelength counterpart. Because of these systematic issues, this search cannot be expected to be complete and it is likely that there are other spatially extended sources that this method missed.

For each candidate that was not biased by neighboring point-like sources or by large-scale residuals in the diffuse emission model, we improved the model of the region by deciding on a case-by-case basis which background point-like sources should be kept. We kept in our model the sources that we believed represented physically distinct sources and we removed sources that we believed were included in the 2FGL catalog to compensate for residuals induced by not modeling the extension of the source. Soft nearby point-like 2FGL sources that were not significant at higher energies were frozen to the spectras predicted by 2FGL. When deciding which background sources to keep and which to remove, we used multiwavelength information about possibly extended source counterparts to help guide our choice. For each extended source presented in Section 10, we describe any modifications from 2FGL of the background model that were performed. In Table 6, we will summarize the sources in the 2FGL catalog that we have concluded here correspond to residuals induced by not modeling the extensions of nearby extended sources.

The best-fit positions of nearby point-like sources can be influenced by the extended source and vice versa. Similarly, the best-fit positions of nearby point-like sources in the 2FGL catalog can be biased by systematic issues at lower energies. Therefore, after selecting the list of background sources, we iteratively refit the positions and spectra of nearby background sources as well as the positions and extensions of the analyzed spatially extended sources until the overall fit converged globally. For each extended source, we will describe the positions of any relocalized background sources.

After obtaining the overall best-fit positions and extensions of all of the sources in the region using pointlike, we refit the spectral parameters of the region using gtlike. With gtlike, we obtained a second measure of $\mathrm{TS}_{\text {ext }}$. We only consider a source to be extended when both pointlike and gtlike agree that $\mathrm{TS}_{\mathrm{ext}} \geqslant 16$. We further required that $\mathrm{TS}_{\mathrm{ext}} \geqslant 16$ using the alternative approach to modeling the diffuse emission presented in Section 8. We then replaced the spatially extended source with two point-like sources and refit the positions and spectra of the two point-like sources to calculate $\mathrm{TS}_{2 \mathrm{pts}}$. We only consider a source to be spatially extended, instead of being the result of confusion of two point-like sources, if $\mathrm{TS}_{\mathrm{ext}}>\mathrm{TS}_{2 \mathrm{pts}}$. As was shown in Section 5, this test is fairly powerful at removing situations in which the emission actually originates from two distinct point-like sources instead of one spatially extended source. On the other hand, it is still possible that longer observations could resolve additional structure or new sources that the analysis cannot currently detect. Considering the very complicated morphologies of extended sources observed at other wavelengths and the high density of possible sources that are expected to emit at $\mathrm{GeV}$ energies, it is likely that in some of these regions further observations will reveal that the emission is significantly more complicated than the simple radially symmetric uniform disk model that we assume.

\section{NEW EXTENDED SOURCES}

Nine extended sources not included in the 2FGL catalog were found by our extended source search. Two of these have been

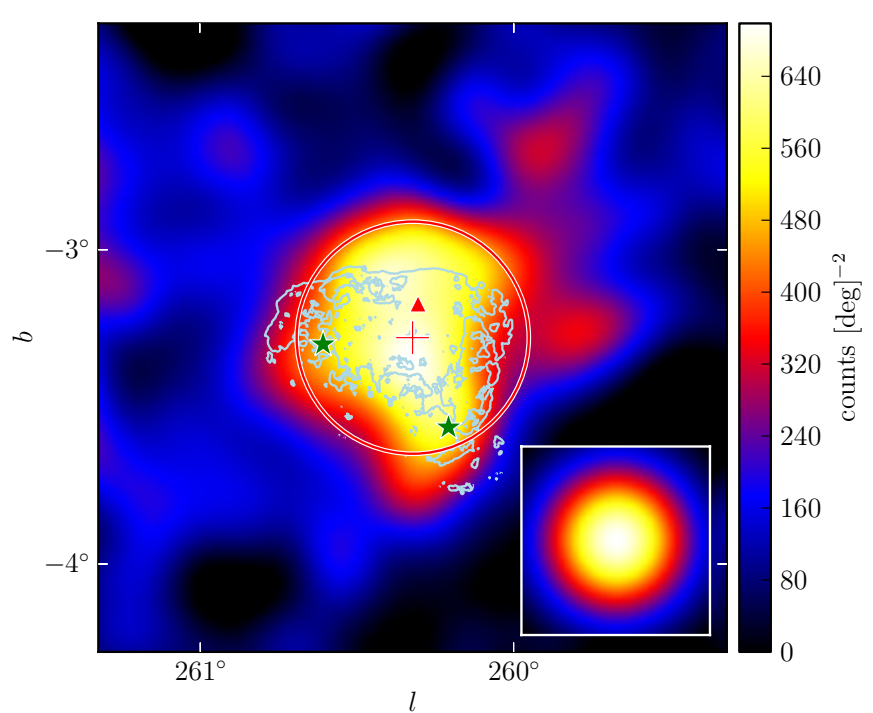

Figure 16. Diffuse-emission-subtracted 1-100 GeV counts map of 2FGL J0823.0-4246 smoothed by a 0.12 D Gaussian kernel. The triangular marker (colored red in the online version) represents the 2FGL position of this source. The plus-shaped marker and the circle (colored red) represent the best-fit position and extension of this source assuming a radially symmetric uniform disk model. The two star-shaped markers (colored green) represent 2FGL sources that were removed from the background model. From left to right, these sources are 2FGL J0823.4-4305 and 2FGL J0821.0-4254. The lower right inset is the model predicted emission from a point-like source with the same spectrum as 2FGL J0823.4-4305 smoothed by the same kernel. This source is spatially coincident with the Puppis A SNR. The light blue contours correspond to the X-ray image of Puppis A observed by ROSAT (Petre et al. 1996).

(A color version of this figure is available in the online journal.)

previously studied in dedicated publications: RX J1713.7-3946 and Vela Jr. (Abdo et al. 2011; Tanaka et al. 2011). Two of these sources were found when using photons with energies between $1 \mathrm{GeV}$ and $100 \mathrm{GeV}$ and seven were found when using photons with energies between $10 \mathrm{GeV}$ and $100 \mathrm{GeV}$. For the sources found at energies above $10 \mathrm{GeV}$, we restrict our analysis to higher energies because of the issues of source confusion and diffuse emission modeling described in Section 9. The spectral and spatial properties of these nine sources are summarized in Table 4 and the results of our investigation of systematic errors are presented in Table 5. Table 5 also compares the likelihood assuming the source is spatially extended to the likelihood assuming that the emission originates from two independent point-like sources. For these new extended sources, $\mathrm{TS}_{\text {ext }}>\mathrm{TS}_{2 \text { pts }}$ so we conclude that the GeV emission does not originate from two physically distinct point-like sources (see Section 5). Table 5 also includes the results of the extension fits using variations of the PSF and the Galactic diffuse model described in Section 8. There is good agreement between $\mathrm{TS}_{\mathrm{ext}}$ and the fit size using the standard analysis, the alternative approach to modeling the diffuse emission, and the alternative PSF. This suggests that the sources are robust against mismodeled features in the diffuse emission model and uncertainties in the PSF.

\subsection{FGL J0823.0-4246}

2FGL J0823.0-4246 was found by our search to be an extended source candidate in the $1-100 \mathrm{GeV}$ energy range and is spatially coincident with the SNR Puppis A. Figure 16 shows a counts map of this source. There are two nearby 2FGL sources 2FGL J0823.4-4305 and 2FGL J0821.0-4254 that are also coincident with the SNR but that do not appear to represent 
Table 4

Extension Fit for the Nine Additional Extended Sources

\begin{tabular}{|c|c|c|c|c|c|c|c|c|c|}
\hline Name & $\begin{array}{c}\text { GLON } \\
(\mathrm{deg})\end{array}$ & $\begin{array}{c}\text { GLAT } \\
(\mathrm{deg})\end{array}$ & $\begin{array}{c}\sigma \\
(\mathrm{deg})\end{array}$ & $\mathrm{TS}$ & $\mathrm{TS}_{\mathrm{ext}}$ & $\begin{array}{l}\text { Pos Err } \\
\text { (deg) }\end{array}$ & Flux $^{a}$ & Index & Counterpart \\
\hline \multicolumn{10}{|c|}{$E>1 \mathrm{GeV}$} \\
\hline 2FGL J0823.0-4246 & 260.32 & -3.28 & $0.37 \pm 0.03 \pm 0.02$ & 322.2 & 48.0 & 0.02 & $8.4 \pm 0.6$ & $2.21 \pm 0.09$ & Puppis A \\
\hline 2FGL J1627.0-2425c & 353.07 & 16.80 & $0.42 \pm 0.05 \pm 0.16$ & 139.9 & 32.4 & 0.04 & $6.3 \pm 0.6$ & $2.50 \pm 0.14$ & Ophiuchus \\
\hline \multicolumn{10}{|c|}{$E>10 \mathrm{GeV}$} \\
\hline 2FGL J0851.7-4635 & 266.31 & -1.43 & $1.15 \pm 0.08 \pm 0.02$ & 116.6 & 86.8 & 0.07 & $1.3 \pm 0.2$ & $1.74 \pm 0.21$ & Vela Jr. \\
\hline 2FGL J1615.0-5051 & 332.37 & -0.13 & $0.32 \pm 0.04 \pm 0.01$ & 50.4 & 16.7 & 0.04 & $1.0 \pm 0.2$ & $2.19 \pm 0.28$ & HESS J1616-508 \\
\hline 2FGL J1615.2-5138 & 331.66 & -0.66 & $0.42 \pm 0.04 \pm 0.02$ & 76.1 & 46.5 & 0.04 & $1.1 \pm 0.2$ & $1.79 \pm 0.26$ & HESS J1614-518 \\
\hline 2FGL J1632.4-4753c & 336.52 & 0.12 & $0.35 \pm 0.04 \pm 0.02$ & 64.4 & 26.9 & 0.04 & $1.4 \pm 0.2$ & $2.66 \pm 0.30$ & HESS J1632-478 \\
\hline 2FGL J1712.4-3941 & 347.26 & -0.53 & $0.56 \pm 0.04 \pm 0.02$ & 59.4 & 38.5 & 0.05 & $1.2 \pm 0.2$ & $1.87 \pm 0.22$ & RX J1713.7-3946 \\
\hline 2FGL J1837.3-0700c & 25.08 & 0.13 & $0.33 \pm 0.07 \pm 0.05$ & 47.0 & 18.5 & 0.07 & $1.0 \pm 0.2$ & $1.65 \pm 0.29$ & HESS J1837-069 \\
\hline 2FGL J2021.5+4026 & 78.24 & 2.20 & $0.63 \pm 0.05 \pm 0.04$ & 237.2 & 128.9 & 0.05 & $2.0 \pm 0.2$ & $2.42 \pm 0.19$ & $\gamma$-Cygni \\
\hline
\end{tabular}

Notes. The columns in this table have the same meaning as those in Table 3. RX J1713.7-3946 and Vela Jr. were previously studied in dedicated publications (Abdo et al. 2011; Tanaka et al. 2011).

${ }^{a}$ Integral flux in units of $10^{-9} \mathrm{ph} \mathrm{cm}^{-2} \mathrm{~s}^{-1}$ and integrated in the fit energy range (either $1-100 \mathrm{GeV}$ or $10-100 \mathrm{GeV}$ ).

b The discrepancy in the best-fit spectra of 2FGL J1712.4-3941 compared to Abdo et al. (2011) is due to fitting over a different energy range.

Table 5

Dual Localization, Alternative PSF, and Alternative Approach to Modeling the Diffuse Emission

\begin{tabular}{|c|c|c|c|c|c|c|c|c|c|c|}
\hline Name & $\mathrm{TS}_{\text {pointlike }}$ & $\mathrm{TS}_{\text {gtlike }}$ & $\mathrm{TS}_{\text {alt, diff }}$ & $\mathrm{TS}_{\text {extpointlike }}$ & $\mathrm{TS}_{\text {extgtlike }}$ & $\mathrm{TS}_{\text {ext alt,diff }}$ & $\begin{array}{c}\sigma \\
(\mathrm{deg})\end{array}$ & $\begin{array}{c}\sigma_{\text {alt, diff }} \\
(\mathrm{deg})\end{array}$ & $\begin{array}{c}\sigma_{\text {alt,psf }} \\
(\mathrm{deg})\end{array}$ & $\overline{\mathrm{TS}_{2 \mathrm{pts}}}$ \\
\hline \multicolumn{11}{|c|}{$E>1 \mathrm{GeV}$} \\
\hline \multicolumn{11}{|c|}{$E>10 \mathrm{GeV}$} \\
\hline 2FGL J0851.7-4635 & 115.2 & 116.6 & 123.1 & 83.9 & 86.8 & 89.8 & 1.15 & 1.16 & 1.17 & 15.5 \\
\hline 2FGL J1632.4-4753c & 64.5 & 64.4 & 66.8 & 23.0 & 26.9 & 25.5 & 0.35 & 0.36 & 0.37 & 10.9 \\
\hline 2FGL J1712.4-3941 & 59.8 & 59.4 & 39.9 & 38.4 & 38.5 & 30.7 & 0.56 & 0.55 & 0.53 & 2.7 \\
\hline 2FGL J1837.3-0700c & 44.5 & 47.0 & 39.2 & 17.6 & 18.5 & 16.1 & 0.33 & 0.32 & 0.38 & 10.8 \\
\hline 2FGL J2021.5+4026 & 239.1 & 237.2 & 255.8 & 139.1 & 128.9 & 138.0 & 0.63 & 0.65 & 0.59 & 37.3 \\
\hline
\end{tabular}

Notes. $\mathrm{TS}_{\text {pointlike }}, \mathrm{TS}_{\text {gtlike }}$, and $\mathrm{TS}_{\text {alt,diff }}$ are the test statistic values from pointlike, gtlike, and gtlike with the alternative approach to modeling the diffuse emission, respectively. $\mathrm{TS}_{\text {extpointlike }}, \mathrm{TS}_{\text {extgtlike }}$, and $\mathrm{TS}_{\text {extalt,diff }}$ are the $\mathrm{TS}$ values from pointlike, gtlike, and gtlike with the alternative approach to modeling the diffuse emission, respectively. $\sigma, \sigma_{\text {alt,diff }}$, and $\sigma_{\text {alt,psf }}$ are the fit sizes assuming a radially symmetric uniform disk model with the standard analysis, the alternative approach to modeling the diffuse emission, and the alternative PSF, respectively.

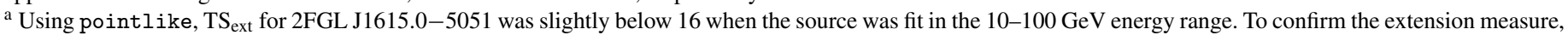
the extension was refit in pointlike using a slightly lower energy. In the 5.6-100 GeV energy range, we obtained a consistent extension and TS $\mathrm{ext}=28.0$. In the rest of this paper, we quote the $E>10 \mathrm{GeV}$ results for consistency with the other sources.

Table 6

Narby Residual-induced Sources

\begin{tabular}{lc}
\hline \hline Extended Source & Residual-induced Sources \\
\hline 2FGL J0823.0-4246 & 2FGL J0821.0-4254, 2FGL J0823.4-4305 \\
2FGL J1627.0-2425c & $\ldots$ \\
2FGL J0851.7-4635 & $\ldots$ \\
2FGL J1615.0-5051 & $\ldots$ \\
2FGL J1615.2-5138 & 2FGL J1614.9-5212 \\
2FGL J1632.4-4753c & 2FGL J1634.4-4743c \\
2FGL J1712.4-3941 & $\ldots$ \\
2FGL J1837.3-0700c & 2FGL J1835.5-0649 \\
2FGL J2021.5+4026 & 2FGL J2019.1+4040 \\
\hline
\end{tabular}

Notes. For each new extended source, we list nearby 2FGL sources that we have concluded here correspond to residuals induced by not modeling the extensions of nearby extended sources. 


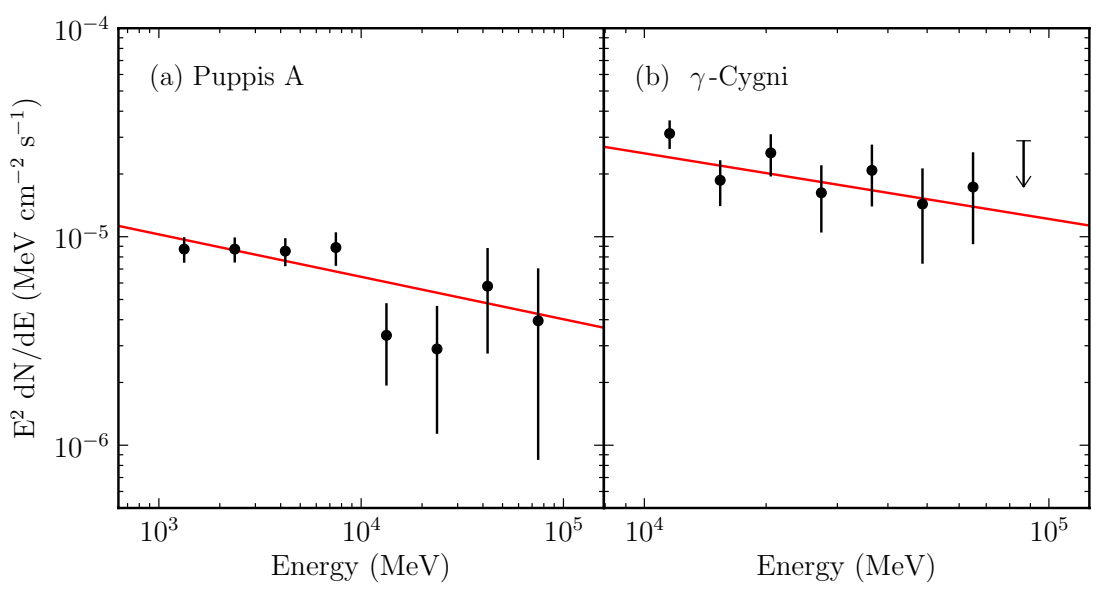

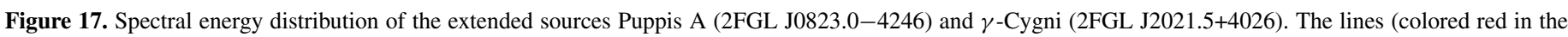

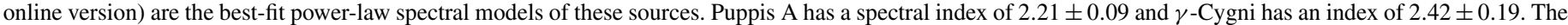
spectral errors are statistical only. The upper limit is at the $95 \%$ confidence level.

(A color version of this figure is available in the online journal.)

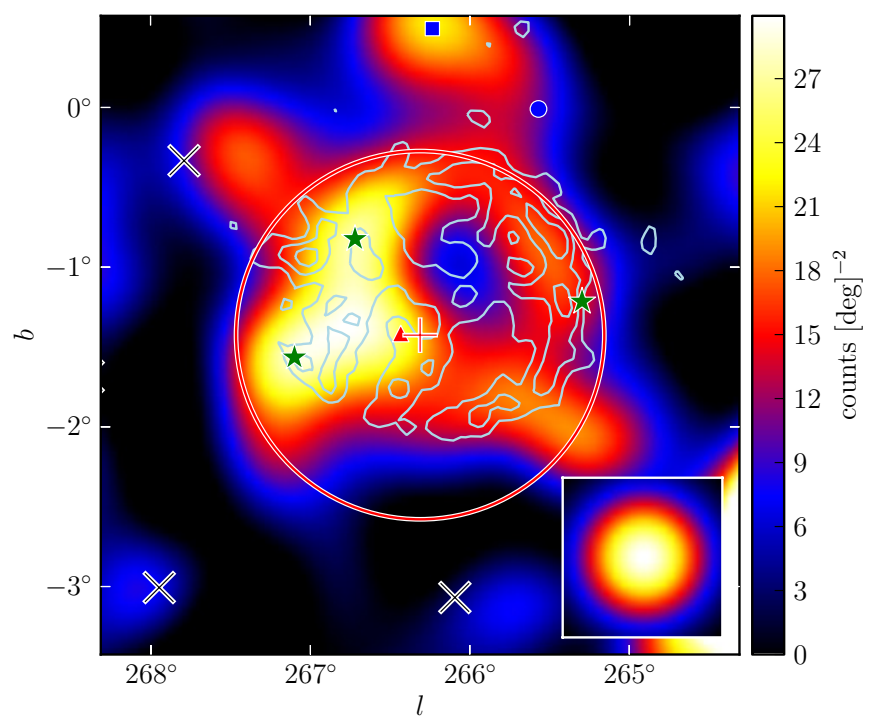

Figure 18. Diffuse-emission-subtracted $10-100 \mathrm{GeV}$ counts map of 2FGL J0851.7-4635 smoothed by a 0.25 2D Gaussian kernel. The triangular marker (colored red in the online version) represents the 2FGL position of this source. The plus-shaped marker and the circle (colored red) are the best-fit position and extension of this source assuming a radially symmetric uniform disk model. The three black crosses represent background 2FGL sources. The three star-shaped markers (colored green) represent other 2FGL sources that were removed from the background model. They are (from left to right) 2FGL J0853.5-4711, 2FGL J0855.4-4625, and 2FGL J0848.5-4535. The circular and square-shaped marker (colored blue) represents the 2FGL and relocalized position of another 2FGL source. This extended source is spatially coincident with the Vela Jr. SNR. The contours (colored light blue) correspond to the TeV image of Vela Jr. (Aharonian et al. 2007a).

(A color version of this figure is available in the online journal.)

physically distinct sources. We conclude that these nearby pointlike sources were included in the 2FGL catalog to compensate for residuals induced by not modeling the extension of this source and removed them from our model of the sky. After removing these sources, 2FGL J0823.0-4246 was found to have an extension $\sigma=0.37 \pm 0.03_{\text {stat }} \pm 0.02_{\text {sys }}$ with $\mathrm{TS}_{\text {ext }}=48.0$. Figure 17 shows the spectrum of this source.

Puppis A has been studied in detail in radio (Castelletti et al. 2006), and X-ray (Petre et al. 1996; Hwang et al. 2008). The fit extension of 2FGL J0823.0-4246 matches well the size of
Puppis A in X-ray. The distance of Puppis A was estimated at $2.2 \mathrm{kpc}$ (Reynoso et al. 1995, 2003) and leads to a $1-100 \mathrm{GeV}$ luminosity of $\sim 3 \times 10^{34} \mathrm{erg} \mathrm{s}^{-1}$. No molecular clouds have been observed directly adjacent to Puppis A (Paron et al. 2008), similar to the LAT-detected Cygnus Loop SNR (Katagiri et al. 2011). The luminosity of Puppis A is also smaller than that of other SNRs believed to interact with molecular clouds (Abdo et al. 2009a, 2010a, 2010f, 2010h, 2010i).

\subsection{FGL J0851.7-4635}

2FGL J0851.7-4635 was found by our search to be an extended source candidate in the $10-100 \mathrm{GeV}$ energy range and is spatially coincident with the SNR Vela Jr. This source was recently studied by the LAT Collaboration in Tanaka et al. (2011). Figure 18 shows a counts map of the source. Overlaid on Figure 18 are TeV contours of Vela Jr. (Aharonian et al. 2007a). There are three point-like 2FGL sources 2FGL J0848.5-4535, 2FGL J0853.5-4711, and 2FGL J0855.4-4625 which correlate with the multiwavelength emission of this SNR but do not appear to be physically distinct sources. They were most likely included in the 2FGL catalog to compensate for residuals induced by not modeling the extension of Vela Jr. and were removed from our model of the sky.

With this model of the background, 2FGL J0851.7-4635 was found to have an extension of $\sigma=1.15 \pm 0.08_{\text {stat }} \pm 0.02_{\text {sys }}$ with $\mathrm{TS}_{\text {ext }}=86.8$. The LAT size matches well the TeV morphology of Vela Jr. While fitting the extension of 2FGL J0851.7-4635, we iteratively relocalized the position of the nearby point-like 2FGL source 2FGL J0854.7-4501 to $(l, b)=(266.24,0.49)$ to better fit its position at high energies.

\subsection{FGL J1615.0-5051}

2FGL J1615.0-5051 and 2FGL J1615.2-5138 were both found to be extended source candidates in the $10-100 \mathrm{GeV}$ energy range. Because they are less than $1^{\circ}$ away from each other, they needed to be analyzed simultaneously. 2FGL J1615.0-5051 is spatially coincident with the extended TeV source HESS J1616-508 and 2FGL J1615.2-5138 is coincident with the extended TeV source HESS J1614-518. Figure 19 shows a counts map of these sources and overlays the TeV contours of HESS J1616-508 and HESS J1614-518 (Aharonian et al. 2006d). The figure shows that the 2FGL source 


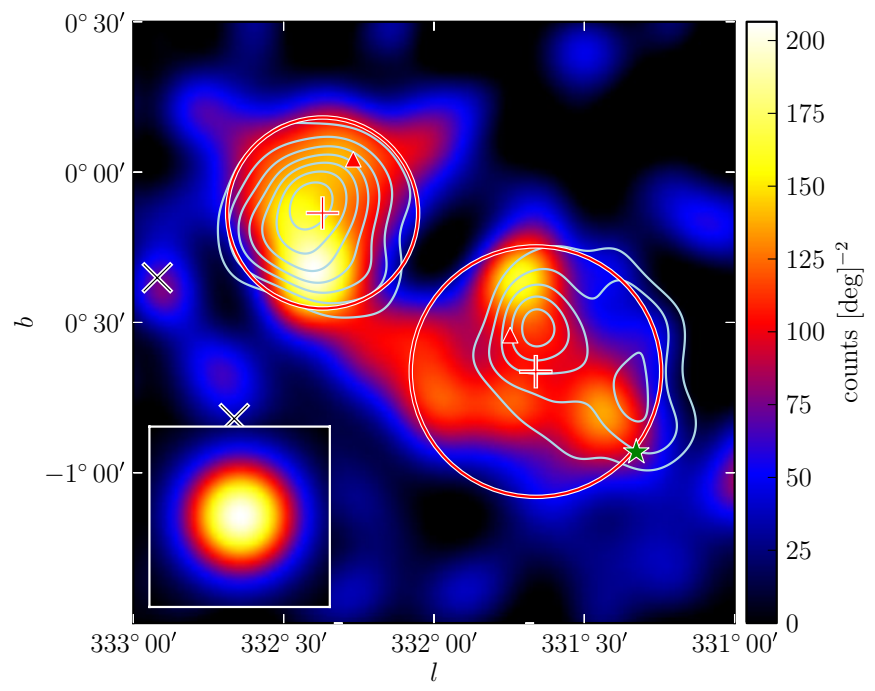

Figure 19. Diffuse-emission-subtracted $10-100 \mathrm{GeV}$ counts map of 2FGL J1615.0-5051 (upper left) and 2FGL J1615.2-5138 (lower right) smoothed by a $0.12 \mathrm{D}$ Gaussian kernel. The triangular markers (colored red in the online version) represent the 2FGL positions of these sources. The crossshaped markers and the circles (colored red) represent the best-fit positions and extensions of these sources assuming a radially symmetric uniform disk model. The two black crosses represent background 2FGL sources and the star-shaped marker (colored green) represents 2FGL J1614.9-5212, another 2FGL source that was removed from the background model. The contours (colored light blue) correspond to the TeV image of HESS J1616-508 (left) and HESS J1614-518 (right) (Aharonian et al. 2006d)

(A color version of this figure is available in the online journal.)
2FGL J1614.9-5212 is very close to 2FGL J1615.2-5138 and correlates with the same extended $\mathrm{TeV}$ source as 2FGL J1615.2-5138. We concluded that this source was included in the 2FGL catalog to compensate for residuals induced by not modeling the extension of 2FGL J1615.2-5138 and removed it from our model of the sky.

With this model of the sky, we iteratively fit the extensions of 2FGL J1615.0-5051 and 2FGL J1615.2-5138. 2FGL J1615.0-5051 was found to have an extension $\sigma=$ $0.32 \pm 0.04_{\text {stat }} \pm 0.01_{\text {sys }}$ and $\mathrm{TS}_{\text {ext }}=16.7$.

The TeV counterpart of 2FGL J1615.0-5051 was fit with a radially symmetric Gaussian surface brightness profile with $\sigma=0.136 \pm 0.008$ (Aharonian et al. 2006d). This TeV size corresponds to a $68 \%$ containment radius of $r_{68}=0.21 \pm 0.01$, comparable to the LAT size $r_{68}=0.26 \pm 0.03$. Figure 20 shows that the spectrum of 2FGL J1615.0-5051 at GeV energies connects to the spectrum of HESS J1616-508 at TeV energies.

HESS J1616-508 is located in the region of two SNRs RCW103 (G332.4-04) and Kes 32 (G332.4+0.1) but is not spatially coincident with either of them (Aharonian et al. 2006d). HESS J1616-508 is near three pulsars PSR J1614-5048, PSR J1616-5109, and PSR J1617-5055 (Torii et al. 1998; Landi et al. 2007a). Only PSR J1617-5055 is energetically capable of powering the $\mathrm{TeV}$ emission and Aharonian et al. (2006d) speculated that HESS J1616-508 could be a PWN powered by this young pulsar. Because HESS J1616-508 is $9^{\prime}$ away from PSR J1617-5055, this would require an asymmetric X-ray PWNe to power the $\mathrm{TeV}$ emission. Chandra ACIS observations revealed an underluminous PWN of size

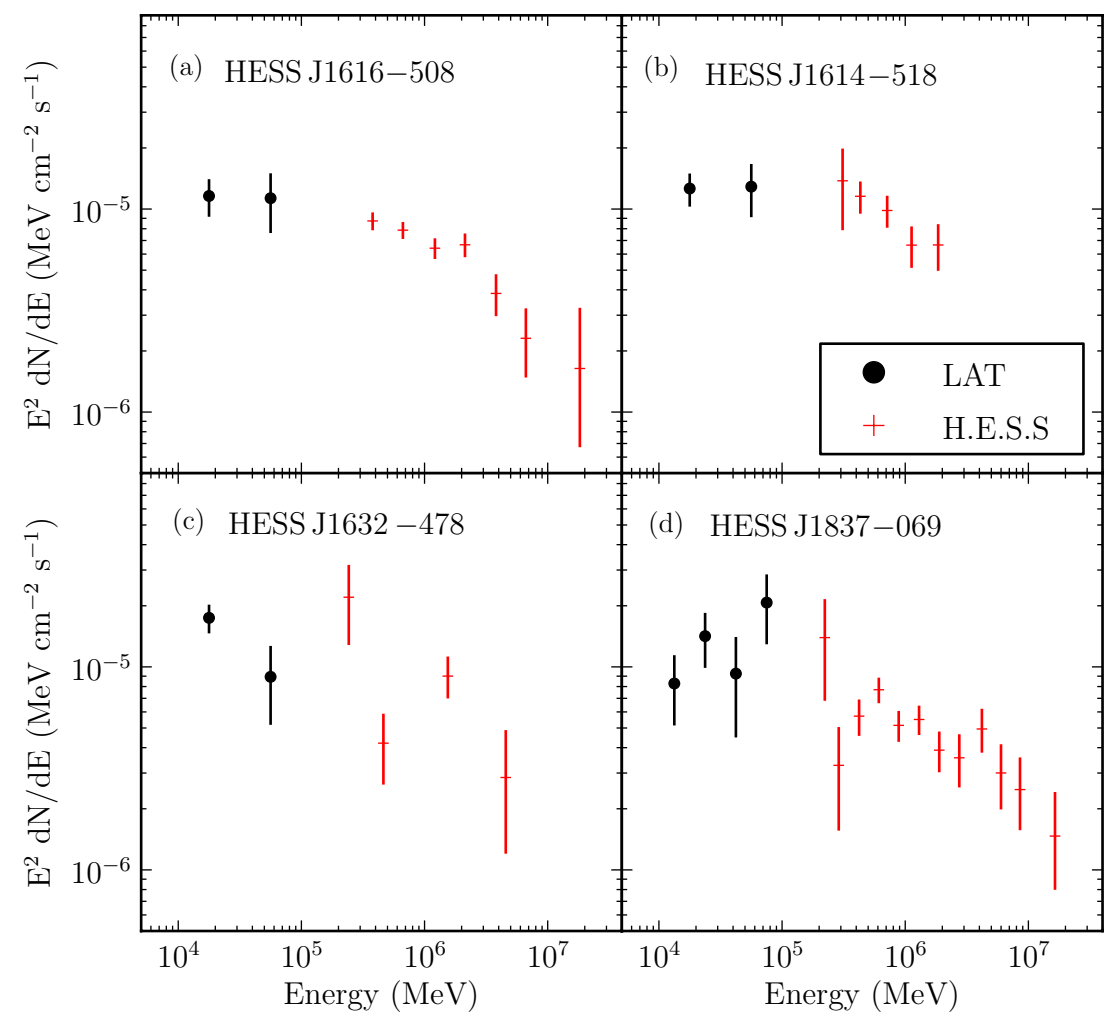

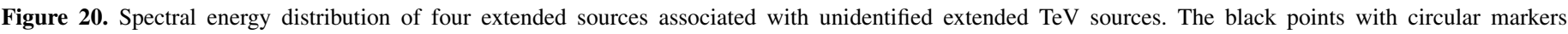

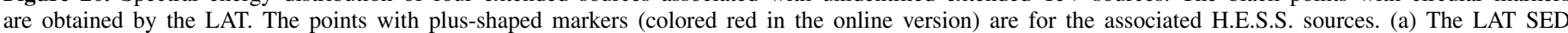

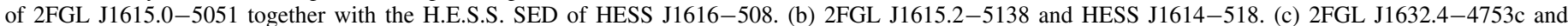

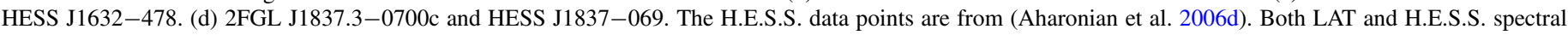
errors are statistical only.

(A color version of this figure is available in the online journal.) 

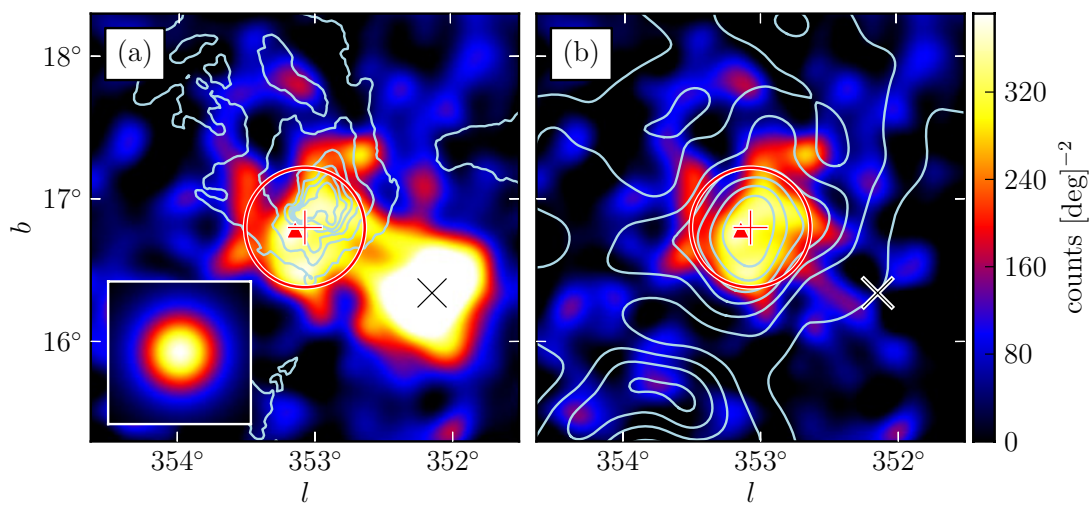

Figure 21. Diffuse-emission-subtracted 1-100 GeV counts map of (a) the region around 2FGL J1627.0-2425 smoothed by a $0.12 \mathrm{D}$ Gaussian kernel and (b) with the emission from 2FGL J1625.7-2526 subtracted. The triangular marker (colored red in the online version) represents the 2FGL position of this source. The plus-shaped marker and the circle (colored red) represent the best-fit position and extension of this source assuming a radially symmetric uniform disk model and the black cross represents a background 2FGL source. The contours in (a) correspond to the $100 \mu \mathrm{m}$ image observed by IRAS (Young et al. 1986). The contours in (b) correspond to $\mathrm{CO}(J=1 \rightarrow 0)$ emission integrated from $-8 \mathrm{~km} \mathrm{~s}^{-1}$ to $20 \mathrm{~km} \mathrm{~s}^{-1}$. They are from de Geus et al. (1990), were cleaned using the moment-masking technique (Dame 2011), and have been smoothed by a 0.25 D Gaussian kernel.

(A color version of this figure is available in the online journal.)

$\sim 1^{\prime}$ around the pulsar that was not oriented toward the $\mathrm{TeV}$ emission, rendering this association uncertain (Kargaltsev et al. 2009). No other promising counterparts were observed at X-ray and soft $\gamma$-ray energies by Suzaku (Matsumoto et al. 2007), Swift/XRT, IBIS/ISGRBI, BeppoSAX, and XMM-Newton (Landi et al. 2007a). Kargaltsev et al. (2009) discovered additional diffuse emission toward the center of HESS J1616-508 using archival radio and infrared observations. Deeper observations will likely be necessary to understand this $\gamma$-ray source.

\section{4. $2 F G L J 1615.2-5138$}

2FGL J1615.2-5138 was found to have an extension $\sigma=$ $0.42 \pm 0.04_{\text {stat }} \pm 0.02_{\text {sys }}$ with $\mathrm{TS}_{\text {ext }}=46.5$. To test for the possibility that 2FGL J1615.2-5138 is not spatially extended but instead composed of two point-like sources (one of them represented in the 2FGL catalog by 2FGL J1614.9-5212), we refit 2FGL J1615.2-5138 as two point-like sources. Because $\mathrm{TS}_{2 \mathrm{pts}}=35.1$ is less than $\mathrm{TS}_{\text {ext }}=46.5$, we conclude that this emission does not originate from two closely spaced point-like sources.

2FGL J1615.2-5138 is spatially coincident with the extended TeV source HESS J1614-518. H.E.S.S. measured a 2D Gaussian extension of $\sigma=0.23 \pm 0.02$ and $\sigma=0.15 \pm 0.02$ in the semi-major and semi-minor axes. This corresponds to a $68 \%$ containment size of $r_{68}=0.35 \pm 0.03$ and $0.23 \pm 0.03$, consistent with the LAT size $r_{68}=0.34 \pm 0.03$. Figure 20 shows that the spectrum of 2FGL J1615.2-5138 at GeV energies connects to the spectrum of HESS J1614-518 at TeV energies. Further data collected by H.E.S.S. in 2007 resolve a double-peaked structure at $\mathrm{TeV}$ energies but no spectral variation across this source, suggesting that the emission is not the confusion of physically separate sources (Rowell et al. 2008). This double-peaked structure is also hinted at in the LAT counts map in Figure 19 but is not very significant. The TeV source was also detected by CANGAROO-III (Mizukami et al. 2011).

There are five nearby pulsars, but none are luminous enough to provide the energy output required to power the $\gamma$-ray emission (Rowell et al. 2008). HESS J1614-518 is spatially coincident with a young open cluster Pismis 22 (Landi et al. 2007b; Rowell et al. 2008). Suzaku detected two promising $\mathrm{X}$-ray candidates. Source A is an extended source consistent with the peak of HESS J1614-518 and source B coincident with Pismis 22 and toward the center but in a relatively dim region of HESS J1614-518 (Matsumoto et al. 2008). Three hypotheses have been presented to explain this emission: either source $\mathrm{A}$ is an SNR powering the $\gamma$-ray emission; source A is a PWN powered by an undiscovered pulsar in either source $\mathrm{A}$ or B; and finally that the emission may arise from hadronic acceleration in the stellar winds of Pismis 22 (Mizukami et al. 2011).

$$
\text { 10.5. 2FGL J1627.0-2425c }
$$

2FGL J1627.0-2425c was found by our search to have an extension $\sigma=0.42 \pm 0.05_{\text {stat }} \pm 0.16_{\text {sys }}$ with $\mathrm{TS}_{\text {ext }}=32.4$ using photons with energies between $1 \mathrm{GeV}$ and $100 \mathrm{GeV}$. Figure 21 shows a counts map of this source.

This source is in a region of remarkably complicated diffuse emission. Even though it is $16^{\circ}$ from the Galactic plane, this source is on top of the core of the Ophiuchus molecular cloud which contains massive star-forming regions that are bright in infrared. The region also has abundant molecular and atomic gas traced by $\mathrm{CO}$ and $\mathrm{H}$ I and significant dark gas found only by its association with dust emission (Grenier et al. 2005). Embedded star-forming regions make it even more challenging to measure the column density of dust. Infrared and $\mathrm{CO}(J=1 \rightarrow 0)$ contours are overlaid on Figure 21 and show good spatial correlation with the $\mathrm{GeV}$ emission (Young et al. 1986; de Geus et al. 1990). This source might represent $\gamma$-ray emission from the interactions of cosmic rays with interstellar gas which has not been accounted for in the LAT diffuse emission model.

$$
\text { 10.6. } 2 F G L J 1632.4-4753 c
$$

2FGL J1632.4-4753c was found by our search to be an extended source candidate in the $10-100 \mathrm{GeV}$ energy range but is in a crowded region of the sky. It is spatially coincident with the TeV source HESS J1632-478. Figure 22(a) shows a counts map of this source and overlays TeV contours of HESS J1632-478 (Aharonian et al. 2006d). There are six nearby point-like 2FGL sources that appear to represent physically distinct sources and were included in our background model: 2FGL J1630.2-4752, 2FGL J1631.7-4720c, 2FGL J1632.4-4820c, 2FGL J1635.4-4717c, 2FGL J1636.34740c, and 2FGL J1638.0-4703c. On the other hand, one pointlike 2FGL source 2FGL J1634.4-4743c correlates with the 

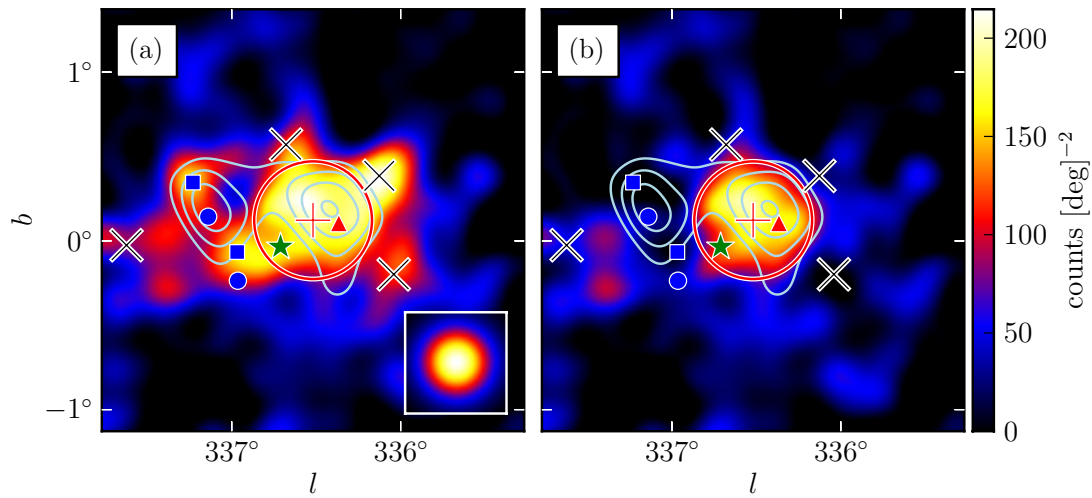

Figure 22. Diffuse-emission-subtracted 10-100 GeV counts map of 2FGL J1632.4-4753c (a) smoothed by a $0.12 \mathrm{D}$ Gaussian kernel and (b) with the emission from the background sources subtracted. The triangular marker (colored red in the online version) represents the 2FGL position of this source. The plus-shaped marker and the circle (colored red) are the best-fit position and extension of 2FGL J1632.4-4753c assuming a radially symmetric uniform disk model. The four black crosses represent background 2FGL sources subtracted in (b). The circular and square-shaped markers (colored blue) represent the 2FGL and relocalized positions, respectively, of two additional background 2FGL sources subtracted in (b). The star-shaped marker (colored green) represents 2FGL J1634.4-4743c, another 2FGL source that was removed from the background model. The contours (colored light blue) correspond to the TeV image of HESS J1632-478 (Aharonian et al. 2006d). (A color version of this figure is available in the online journal.)

extended $\mathrm{TeV}$ source and at $\mathrm{GeV}$ energies does not appear physically separate. It is very close to the position of 2FGL J1632.4-4753c and does not show spatially separated emission in the observed photon distribution. We therefore removed this source from our model of the background. Figure 22(b) shows the same region with the background sources subtracted. With this model, 2FGL J1632.4-4753c was found to have an extension $\sigma=0.35 \pm 0.04_{\text {stat }} \pm 0.02_{\text {sys }}$ with $\mathrm{TS}_{\text {ext }}=$ 26.9. While fitting the extension of 2FGL J1632.4-4753c, we iteratively relocalized 2FGL J1635.4-4717c to $(l, b)=$ $(337.23,0.35)$ and $2 \mathrm{FGL} \mathrm{J} 1636.3-4740 \mathrm{c}$ to $(l, b)=$ (336.97, -0.07$)$.

H.E.S.S. measured an extension of $\sigma=0.21 \pm 0.05$ and $0.06 \pm 0.04$ along the semi-major and semi-minor axes when fitting HESS J1632-478 with an elliptical 2D Gaussian surface brightness profile. This corresponds to a $68 \%$ containment size $r_{68}=0.31 \pm 0.08$ and $0.09 \pm 0.06$ along the semimajor and semi-minor axes, consistent with the LAT size $r_{68}=0.29 \pm 0.04$. Figure 20 shows that the spectrum of 2FGL J1632.4-4753c at GeV energies connects to the spectrum of HESS J1632-478 at TeV energies.

Aharonian et al. (2006d) argued that HESS J1632-478 is positionally coincident with the hard X-ray source IGR J1632-4751 observed by ASCA, International GammaRay Astrophysics Laboratory, and XMM-Newton (Sugizaki et al. 2001; Tomsick et al. 2003; Rodriguez et al. 2003), but this source is suspected to be a Galactic X-Ray Binary so the $\gamma$-ray extension disfavors the association. Further observations by $X M M$-Newton discovered point-like emission coincident with the peak of the H.E.S.S. source surrounded by extended emission of size $\sim 32^{\prime \prime} \times 15^{\prime \prime}$ (Balbo et al. 2010). They found in archival MGPS-2 data a spatially coincident extended radio source (Murphy et al. 2007) and argued for a single synchrotron and IC process producing the radio, X-ray, and $\mathrm{TeV}$ emission, likely due to a PWN. The increased size at TeV energies compared to X-ray energies has previously been observed in several aging PWNe including HESS J1825-137 (Gaensler et al. 2003; Aharonian et al. 2006b), HESS J1640-465 (Aharonian et al. 2006d; Funk et al. 2007), and Vela X (Markwardt \& Ogelman 1995; Aharonian et al. 2006c) and can be explained by different synchrotron cooling times for the electrons that produce $\mathrm{X}$-rays and $\gamma$-rays.

\subsection{FGL J1712.4-3941}

2FGL J1712.4-3941 was found by our search to be spatially extended using photons with energies between $1 \mathrm{GeV}$ and $100 \mathrm{GeV}$. This source is spatially coincident with the SNR RX J1713.7-3946 and was recently studied by the LAT Collaboration in Abdo et al. (2011). To avoid issues related to uncertainties in the nearby Galactic diffuse emission at lower energy, we restricted our analysis only to energies above $10 \mathrm{GeV}$. Figure 23 shows a smoothed counts map of the source. Above $10 \mathrm{GeV}$, the $\mathrm{GeV}$ emission nicely correlates with the $\mathrm{TeV}$ contours of RX J1713.7-3946 (Aharonian et al. 2007b) and 2FGL J1712.4-3941 fit to an extension $\sigma=0.56 \pm 0.04_{\text {stat }} \pm$ $0.02_{\text {sys }}$ with $\mathrm{TS}_{\mathrm{ext}}=38.5$.

\subsection{FGL J1837.3-0700c}

2FGL J1837.3-0700c was found by our search to be an extended source candidate in the $10-100 \mathrm{GeV}$ energy range and is spatially coincident with the $\mathrm{TeV}$ source HESS J1837-069. This source is in a complicated region. Figure 24(a) shows a smoothed counts map of the region and overlays the TeV contours of HESS J1837-069 (Aharonian et al. 2006d). There are two very nearby point-like 2FGL sources, 2FGL J1836.8-0623c and 2FGL J1839.3-0558c, that clearly represent distinct sources. On the other hand, there is another source 2FGL J1835.5-0649 located between the three sources that appears to correlate with the TeV morphology of HESS J1837-069 but at GeV energies does not appear to represent a physically distinct source. We concluded that this source was included in the 2FGL catalog to compensate for residuals induced by not modeling the extension of this source and removed it from our model of the sky. Figure 24(b) shows a counts map of this region after subtracting these background sources. After removing 2FGL J1835.5-0649, we tested for source confusion by fitting 2FGL J1837.3-0700c instead as two point-like sources. Because $\mathrm{TS}_{2 \text { pts }}=10.8$ is less than $\mathrm{TS}_{\mathrm{ext}}=18.5$, we conclude that this emission does not originate from two nearby point-like sources.

With this model, 2FGL J1837.3-0700c was found to have an extension $\sigma=0.33 \pm 0.07_{\text {stat }} \pm 0.05_{\text {sys }}$. While fitting the extension of 2FGL J1837.3-0700c, we iteratively relocalized 

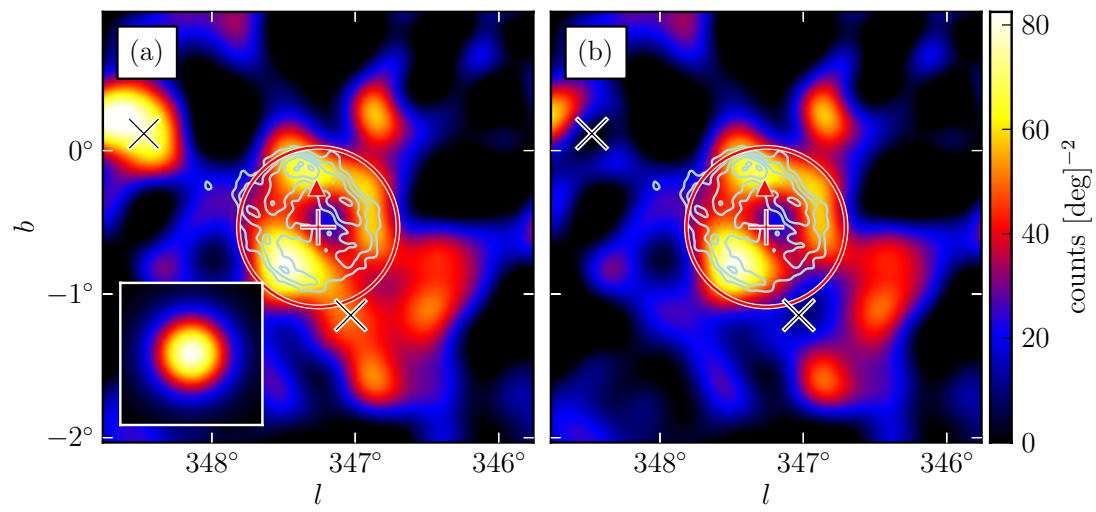

Figure 23. Diffuse-emission-subtracted 10-100 GeV counts map of 2FGL J1712.4-3941 (a) smoothed by a 0.15 2D Gaussian kernel and (b) with the emission from the background sources subtracted. This source is spatially coincident with RX J1713.7-3946 and was recently studied in Abdo et al. (2011). The triangular marker (colored red in the online version) represents the 2FGL position of this source. The plus-shaped marker and the circle (colored red) are the best-fit position and extension of this source assuming a radially symmetric uniform disk model. The two black crosses represent background 2FGL sources subtracted in (b). The contours (colored light blue) correspond to the TeV image (Aharonian et al. 2007b).

(A color version of this figure is available in the online journal.)
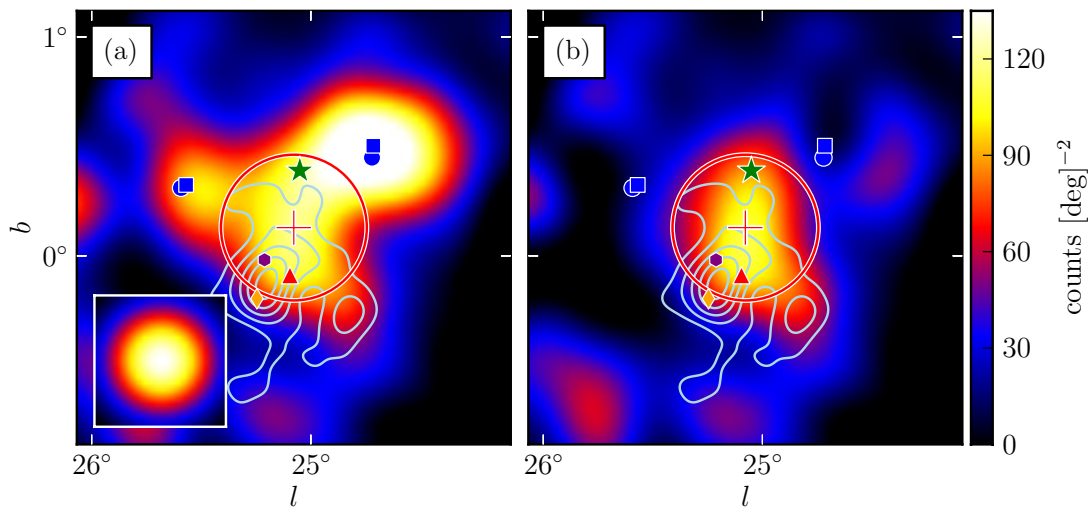

Figure 24. Diffuse-emission-subtracted $10-100 \mathrm{GeV}$ counts map of the region around 2FGL J1837.3-0700c (a) smoothed by a 0.15 2D Gaussian kernel and (b) with the emission from the background sources subtracted. The triangular marker (colored red in the online version) represents the 2FGL position of this source. The plus-shaped marker and the circle (colored red) represent the best-fit position and extension of 2FGL J1837.3-0700c assuming a radially symmetric uniform disk model. The circular and square-shaped markers (colored blue) represent the 2FGL and the relocalized positions, respectively, of two background 2FGL sources subtracted in (b). The star-shaped marker (colored green) represents 2FGL J1835.5-0649, another 2FGL source that was removed from the background model. The contours (colored light blue) correspond to the TeV image of HESS J1837-069 (Aharonian et al. 2006d). The diamond-shaped marker (colored orange) represents the position of PSR J1838-0655 and the hexagonal-shaped marker (colored purple) represents the position AX J1837.3-0652 (Gotthelf \& Halpern 2008).

(A color version of this figure is available in the online journal.)

the two closest background sources along with the extension of 2FGL J1837.3-0700c but their positions did not significantly change. 2FGL J1834.7-0705c moved to $(l, b)=(24.77,0.50)$, 2FGL J1836.8-0623c moved to $(l, b)=(25.57,0.32)$.

H.E.S.S. measured an extension of $\sigma=0.12 \pm 0.02$ and $0.05 \pm 0.02$ of the coincident TeV source HESS J1837-069 along the semi-major and semi-minor axes when fitting this source with an elliptical 2D Gaussian surface brightness profile. This corresponds to a $68 \%$ containment radius of $r_{68}=$ $0.18 \pm 0.03$ and $0.08 \pm 0.03$ along the semi-major and semiminor axes. The size is not significantly different from the LAT $68 \%$ containment radius of $r_{68}=0.27 \pm 0.07$ (less than $2 \sigma$ ). Figure 20 shows that the spectrum of 2FGL J1837.3-0700c at $\mathrm{GeV}$ energies connects to the spectrum of HESS J1837-069 at $\mathrm{TeV}$ energies.

HESS J1837-069 is coincident with the hard and steady X-ray source AX J1838.0-0655 (Bamba et al. 2003). This source was discovered by RXTE to be a pulsar (PSR $\mathrm{J} 1838-0655$ ) sufficiently luminous to power the TeV emission and was resolved by Chandra to be a bright point-like source surrounded by a $\sim 2^{\prime}$ nebula (Gotthelf \& Halpern 2008). The $\gamma$-ray emission may be powered by this pulsar. The hard spectral index and spatial extension of 2FGL J1837.3-0700c disfavor a pulsar origin of the LAT emission and suggest instead that the $\mathrm{GeV}$ and $\mathrm{TeV}$ emission both originate from the pulsar's wind. There is another X-ray point-like source AX J1837.3-0652 near HESS J1837-069 (Bamba et al. 2003) that was also resolved into a point-like and diffuse component (Gotthelf \& Halpern 2008). Although no pulsations have been detected from it, it could also be a pulsar powering some of the $\gamma$-ray emission.

\section{9. $2 F G L J 2021.5+4026$}

The source 2FGL J2021.5+4026 is associated with the $\gamma$-Cygni SNR and has been speculated to originate from the interaction of accelerated particles in the SNR with dense molecular clouds (Pollock 1985; Gaisser et al. 1998). This association was disfavored when the $\mathrm{GeV}$ emission from this source was detected to be pulsed (PSR J2021+4026; Abdo et al. 20101). This pulsar was also observed by AGILE (Chen et al. 2011).

Looking at the same region at energies above $10 \mathrm{GeV}$, the pulsar is no longer significant but we instead found in our search 


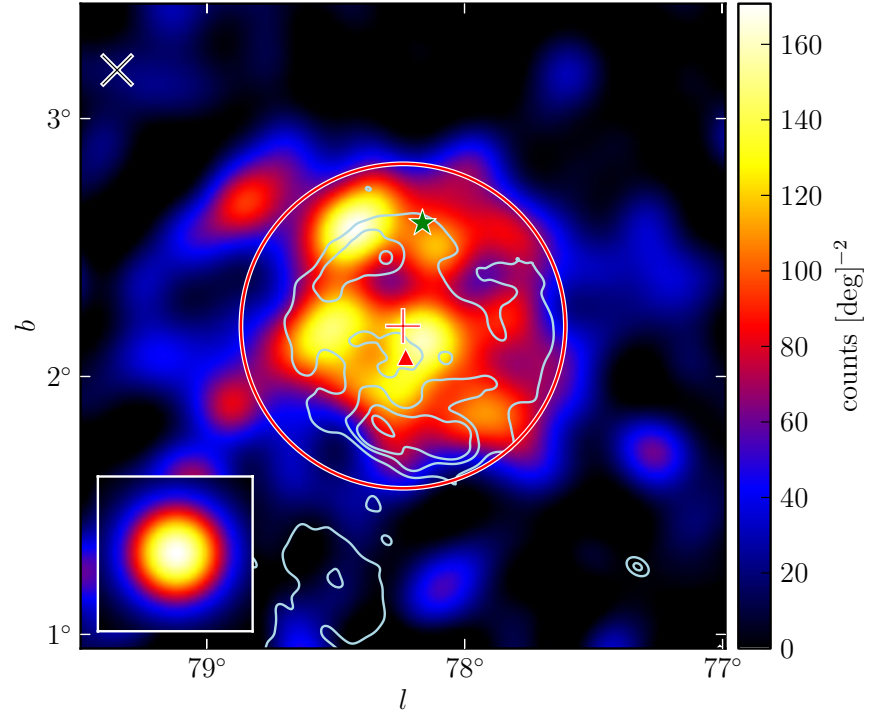

Figure 25. Diffuse-emission-subtracted 10-100 GeV counts map of the region around 2FGL J2021.5+4026 smoothed by a $0.12 \mathrm{D}$ Gaussian kernel. The triangular marker (colored red in the online version) represents the 2FGL position of this source. The plus-shaped marker and the circle (colored red) represent the best-fit position and extension of 2FGL J2021.5+4026 assuming a radially symmetric uniform disk model. The star-shaped marker (colored green) represents 2 FGL J2019.1+4040, a 2FGL source that was removed from the background model. 2FGL J2021.5+4026 is spatially coincident with the $\gamma$-Cygni SNR. The contours (colored light blue) correspond to the $408 \mathrm{MHz}$ image of $\gamma$-Cygni observed by the Canadian Galactic Plane Survey (Taylor et al. 2003).

(A color version of this figure is available in the online journal.)

an extended source candidate. Figure 25 shows a counts map of this source and overlays radio contours of $\gamma$-Cygni from the Canadian Galactic Plane Survey (Taylor et al. 2003). There is good spatial overlap between the SNR and the GeV emission.

There is a nearby source 2FGL J2019.1+4040 that correlates with the radio emission of $\gamma$-Cygni and at $\mathrm{GeV}$ energies does not appear to represent a physically distinct source. We concluded that it was included in the 2FGL catalog to compensate for residuals induced by not modeling the extension of $\gamma$-Cygni and removed it from our model of the sky. With this model,
2FGL J2021.5+4026 was found to have an extension $\sigma=$ $0.63 \pm 0.05_{\text {stat }} \pm 0.04_{\text {sys }}$ with $\mathrm{TS}_{\text {ext }}=128.9$. Figure 17 shows its spectrum. The inferred size of this source at $\mathrm{GeV}$ energies well matches the radio size of $\gamma$-Cygni. Milagro detected a $4.2 \sigma$ excess at energies $\sim 30 \mathrm{TeV}$ from this location (Abdo et al. 2009b, 2009d). VERITAS also detected an extended source VER J2019+407 coincident with the SNR above $200 \mathrm{GeV}$ and suggested that the $\mathrm{TeV}$ emission could be a shock-cloud interaction in $\gamma$-Cygni (Weinstein 2009).

\section{DISCUSSION}

Twelve extended sources were included in the 2FGL catalog and two additional extended sources were studied in dedicated publications. Using two years of LAT data and a new analysis method, we presented the detection of seven additional extended sources. We also reanalyzed the spatial extents of the 12 extended sources in the 2FGL catalog and the two additional sources. The 21 extended LAT sources are located primarily along the Galactic plane and their locations are shown in Figure 26. Most of the LAT-detected extended sources are expected to be of Galactic origin as the distances of extragalactic sources (with the exception of the local group Galaxies) are typically too large to be able to resolve them at $\gamma$-ray energies.

For the LAT extended sources also seen at TeV energies, Figure 27 shows that there is a good correlation between the sizes of the sources at $\mathrm{GeV}$ and $\mathrm{TeV}$ energies. Even so, the sizes of PWNe are expected to vary across the GeV and TeV energy range and the size of HESS J1825-137 is significantly larger at $\mathrm{GeV}$ than $\mathrm{TeV}$ energies (Grondin et al. 2011). It is interesting to compare the sizes of other PWN candidates at $\mathrm{GeV}$ and $\mathrm{TeV}$ energies, but definitively measuring a difference in size would require a more in-depth analysis of the LAT data using the same elliptical Gaussian spatial model.

Figure 28 compares the sizes of the 21 extended LAT sources to the 42 extended H.E.S.S. sources. ${ }^{16}$ Because of the large field of view and all-sky coverage, the LAT can more easily measure

16 The TeV extension of the 42 extended H.E.S.S. sources comes from the H.E.S.S. Source Catalog http://www.mpi-hd.mpg.de/hfm/HESS/pages/ home/sources/.

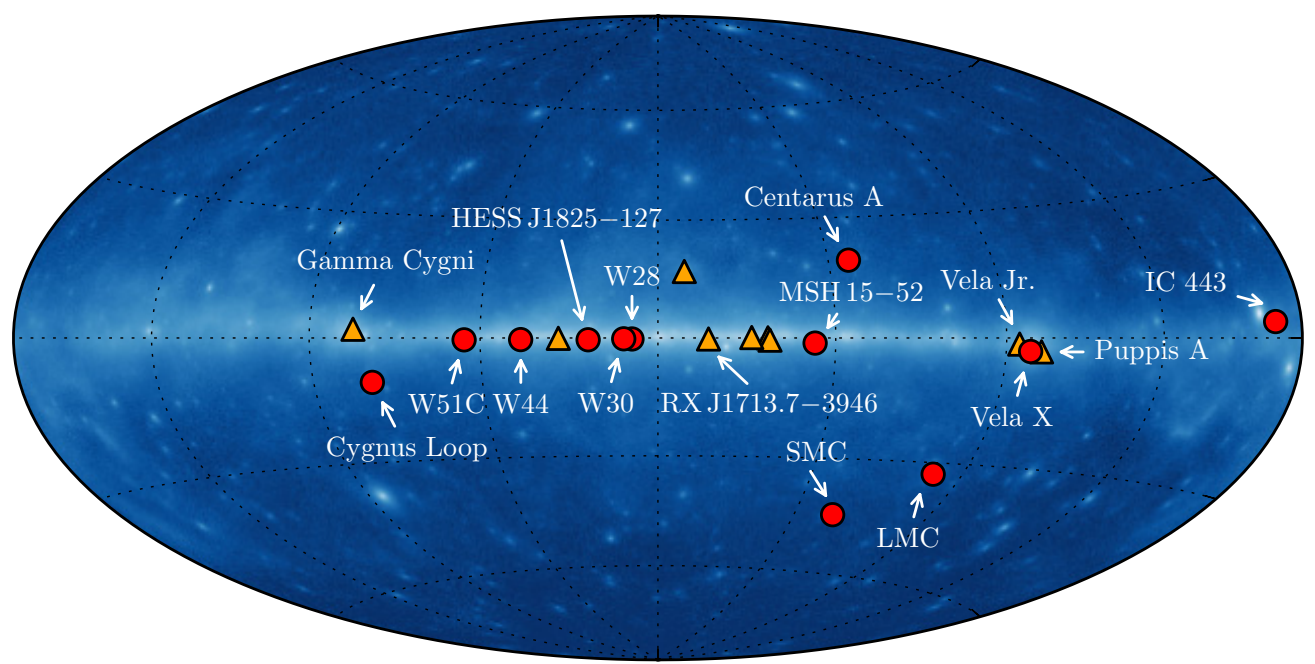

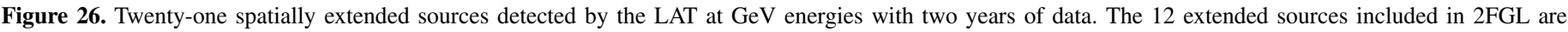

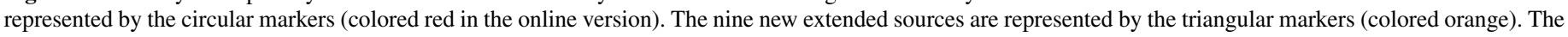
source positions are overlaid on a $100 \mathrm{MeV}$ to $100 \mathrm{GeV}$ Aitoff projection sky map of the LAT data in Galactic coordinates.

(A color version of this figure is available in the online journal.) 


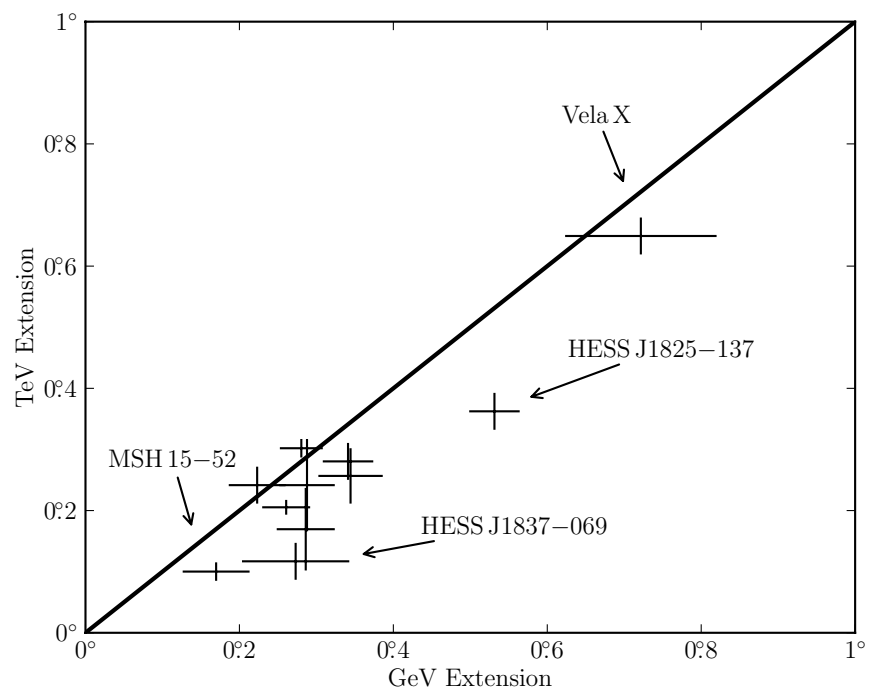

Figure 27. Comparison of the sizes of extended sources detected at both $\mathrm{GeV}$ and $\mathrm{TeV}$ energies. The $\mathrm{TeV}$ sizes of W30, 2FGL J1837.3-0700c, 2FGL J1632.4-4753c, 2FGL J1615.0-5051, and 2FGL J1615.2-5138 are from Aharonian et al. (2006d). The TeV sizes of MSH 15-52, HESS J1825-137, Vela X, Vela Jr., RX J1713.7-3946, and W28 are from Aharonian et al. (2005, 2006b, 2006c, 2007a, 2007b, 2008). The TeV size of IC 443 is from Acciari et al. (2009) and W51C is from Krause et al. (2011). The TeV sizes of MSH 15-52, HESS J1614-518, HESS J1632-478, and HESS J1837-069 have only been reported with an elliptical 2D Gaussian fit and so the plotted sizes are the geometric mean of the semi-major and semiminor axes. The LAT extension of Vela $X$ is from Abdo et al. (2010g). The $\mathrm{TeV}$ sources were fit assuming a 2D Gaussian surface brightness profile so the plotted $\mathrm{GeV}$ and $\mathrm{TeV}$ extensions were first converted to $r_{68}$ (see Section 2.4). Because of their large sizes, the shapes of RX J1713.7-3946 and Vela Jr. were not directly fit at $\mathrm{TeV}$ energies and so are not included in this comparison. On the other hand, dedicated publications by the LAT Collaboration on these sources showed that their morphologies are consistent (Abdo et al. 2011; Tanaka et al. 2011). The LAT extension errors are the statistical and systematic errors added in quadrature.

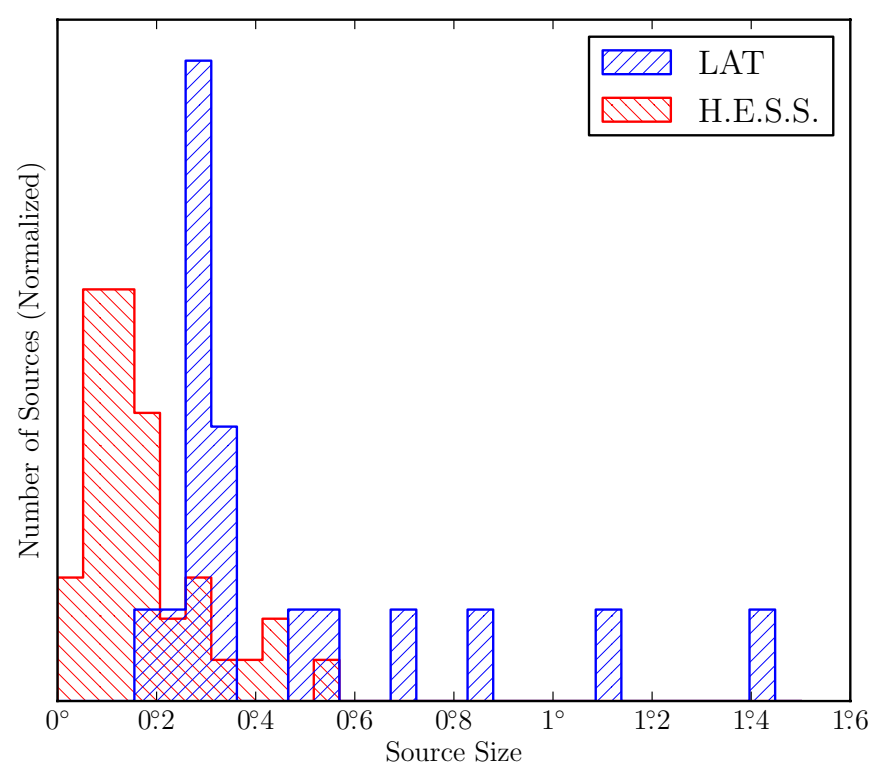

Figure 28. Distributions of the sizes of 18 extended LAT sources at $\mathrm{GeV}$ energies (colored blue in the online version) and the sizes of the 40 extended H.E.S.S. sources at $\mathrm{TeV}$ energies (colored red). The H.E.S.S. sources were fit with a 2D Gaussian surface brightness profile so the LAT and H.E.S.S. sizes were first converted to $r_{68}$. The $\mathrm{GeV}$ size of Vela $\mathrm{X}$ is taken from Abdo et al. (2010g). Because of their large sizes, the shapes of RX J1713.7-3946 and Vela Jr. were not directly fit at $\mathrm{TeV}$ energies and are not included in this comparison. Centaurus A is not included because of its large size.

(A color version of this figure is available in the online journal.)

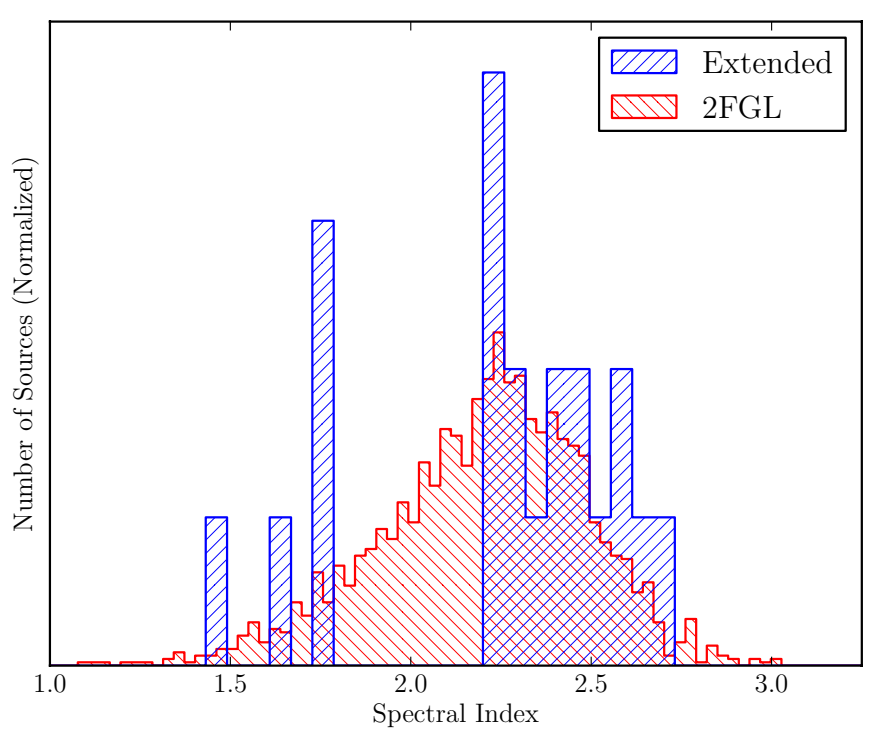

Figure 29. Distribution of spectral indices of the 1873 2FGL sources (colored red in the online version) and the 21 spatially extended sources (colored blue). The index of Centaurus A is taken from Nolan et al. (2012) and the index of Vela $\mathrm{X}$ is taken from Abdo et al. (2010g).

(A color version of this figure is available in the online journal.)

larger sources. On the other hand, the better angular resolution of air Cerenkov detectors allows them to measure a population of extended sources below the resolution limit of the LAT (currently about $\sim 0.2$ ). Fermi has a five-year nominal mission lifetime with a goal of 10 years of operation. As Figure 10 shows, the low background of the LAT at high energies allows its sensitivity to these smaller sources to improve by a factor greater than the square root of the relative exposures. With increasing exposure, the LAT will likely begin to detect and resolve some of these smaller $\mathrm{TeV}$ sources.

Figure 29 compares the spectral indices of LAT-detected extended sources and of all sources in the 2FGL cata$\log$. This, and Tables 3 and 4, shows that the LAT observes a population of hard extended sources at energies above $10 \mathrm{GeV}$. Figure 20 shows that the spectra of four of these sources (2FGL J1615.0-5051, 2FGL J1615.2-5138, 2FGL J1632.4-4753c, and 2FGL J1837.3-0700c) at GeV energies connects to the spectra of their H.E.S.S. counterparts at $\mathrm{TeV}$ energies. This is also true of Vela Jr., HESS J1825-137 (Grondin et al. 2011), and RX J1713.7-3946 (Abdo et al. 2011). It is likely that the $\mathrm{GeV}$ and $\mathrm{TeV}$ emission from these sources originates from the same population of high-energy particles.

Many of the TeV-detected extended sources now seen at $\mathrm{GeV}$ energies are currently unidentified and further multiwavelength follow-up observations will be necessary to understand these particle accelerators. Extending the spectra of these $\mathrm{TeV}$ sources toward lower energies with LAT observations may help to determine the origin and nature of the high-energy emission.

The Fermi-LAT Collaboration acknowledges generous ongoing support from a number of agencies and institutes that have supported both the development and the operation of the LAT as well as scientific data analysis. These include the National Aeronautics and Space Administration and the Department of Energy in the United States, the Commissariat à l'Energie Atomique and the Centre National de la Recherche Scientifique/Institut National de Physique Nucléaire et de Physique des Particules in France, the Agenzia Spaziale Italiana and the Istituto Nazionale 
di Fisica Nucleare in Italy, the Ministry of Education, Culture, Sports, Science, and Technology (MEXT), High Energy Accelerator Research Organization (KEK), and Japan Aerospace Exploration Agency (JAXA) in Japan, and the K. A. Wallenberg Foundation, the Swedish Research Council, and the Swedish National Space Board in Sweden.

Additional support for science analysis during the operations phase is gratefully acknowledged from the Istituto Nazionale di Astrofisica in Italy and the Centre National d'Études Spatiales in France.

This research has made use of pywcsgrid2, an open-source plotting package for Python. ${ }^{17}$ The authors acknowledge the use of HEALPix ${ }^{18}$ (Górski et al. 2005).

\section{REFERENCES}

Abdo, A. A., Ackermann, M., Ajello, M., et al. 2009a, ApJ, 706, L1 Abdo, A. A., Ackermann, M., Ajello, M., et al. 2009b, ApJS, 183, 46 Abdo, A. A., Ackermann, M., Ajello, M., et al. 2009c, Astropart. Phys., 32, 193 Abdo, A., Ackermann, M., Ajello, M., Baldini, L., Ballet, J., et al. 2010a, ApJ, 722,1303

Abdo, A. A., Ackermann, M., Ajello, M., et al. 2010b, ApJ, 714, 927

Abdo, A. A., Ackermann, M., Ajello, M., et al. 2010c, A\&A, 523, A46

Abdo, A. A., Ackermann, M., Ajello, M., et al. 2010d, Science, 328, 725

Abdo, A. A., Ackermann, M., Ajello, M., et al. 2010e, ApJS, 188, 405

Abdo, A. A., Ackermann, M., Ajello, M., et al. 2010f, ApJ, 718, 348

Abdo, A. A., Ackermann, M., Ajello, M., et al. 2010g, ApJ, 713, 146

Abdo, A. A., Ackermann, M., Ajello, M., et al. 2010h, Science, 327, 1103

Abdo, A. A., Ackermann, M., Ajello, M., et al. 2010i, ApJ, 712, 459

Abdo, A. A., Ackermann, M., Ajello, M., et al. 2010j, A\&A, 512, A7

Abdo, A. A., Ackermann, M., Ajello, M., et al. 2010k, Phys. Rev. Lett., 104,101101

Abdo, A. A., Ackermann, M., Ajello, M., et al. 20101, ApJS, 187, 460

Abdo, A. A., Ackermann, M., Ajello, M., et al. 2011, ApJ, 734, 28

Abdo, A. A., Ackermann, M., Ajello, M., et al. 2012a, arXiv:1206.1896

Abdo, A. A., Ackermann, M., Ajello, M., et al. 2012b, ApJ, 744, 80

Abdo, A. A., Allen, B. T., Aune, T., et al. 2009d, ApJ, 700, L127

Acciari, V. A., Aliu, E., Arlen, T., et al. 2009, ApJ, 698, L133

Acciari, V. A., Aliu, E., Arlen, T., et al. 2011, ApJ, 738, 3

Ackermann, M., Ajello, M., Allafort, A., et al. 2011, ApJ, 743, 171

Aharonian, F., Akhperjanian, A. G., Aye, K.-M., et al. 2005, A\&A, 435, L17

Aharonian, F., Akhperjanian, A. G., Bazer-Bachi, A. R., et al. 2006a, A\&A, 460,743

Aharonian, F., Akhperjanian, A. G., Bazer-Bachi, A. R., et al. 2006b, A\&A, 460,365

Aharonian, F., Akhperjanian, A. G., Bazer-Bachi, A. R., et al. 2006c, A\&A, 448, L43

Aharonian, F., Akhperjanian, A. G., Bazer-Bachi, A. R., et al. 2006d, ApJ, 636, 777

Aharonian, F., Akhperjanian, A. G., Bazer-Bachi, A. R., et al. 2007a, ApJ, 661,236

Aharonian, F., Akhperjanian, A. G., Bazer-Bachi, A. R., et al. 2007b, A\&A, 464, 235

Aharonian, F. A., Akhperjanian, A. G., Bazer-Bachi, A. R., et al. 2007c, A\&A, 469, L1

Aharonian, F., Akhperjanian, A. G., Bazer-Bachi, A. R., et al. 2008, A\&A, 481,401

Aharonian, F. A., Coppi, P. S., \& Voelk, H. J. 1994, ApJ, 423, L5

Akaike, H. 1974, IEEE Trans. Autom. Control, 19, 716

Albert, J., Aliu, E., Anderhub, H., et al. 2006, Science, 312, 1771

Atwood, W. B., Abdo, A. A., Ackermann, M., et al. 2009, ApJ, 697, 1071

Balbo, M., Saouter, P., Walter, R., et al. 2010, A\&A, 520, A111
Baltz, E. A., Berenji, B., Bertone, G., et al. 2008, J. Cosmol. Astropart. Phys., JCAP07(2008)013

Bamba, A., Ueno, M., Koyama, K., \& Yamauchi, S. 2003, ApJ, 589, 253

Burnham, K. P., \& Anderson, D. R. 2002, Model Selection and Multimodel Inference: A Practical Information-theoretic Approach (2nd ed.; Berlin: Springer), 1

Castelletti, G., Dubner, G., Golap, K., \& Goss, W. M. 2006, A\&A, 459, 535

Chen, A. W., Piano, G., Tavani, M., et al. 2011, A\&A, 525, A33

Dame, T. M. 2011, arXiv:1101.1499

de Geus, E. J., Bronfman, L., \& Thaddeus, P. 1990, A\&A, 231, 137

Eadie, W. T., Drijard, D., \& James, F. E. (ed.) 1971, Statistical Methods in Experimental Physics (Amsterdam: North-Holland)

Funk, S., Hinton, J. A., Pühlhofer, G., et al. 2007, ApJ, 662, 517

Gaensler, B. M., Schulz, N. S., Kaspi, V. M., Pivovaroff, M. J., \& Becker, W. E. 2003, ApJ, 588, 441

Gaisser, T. K., Protheroe, R. J., \& Stanev, T. 1998, ApJ, 492, 219

Giordano, F. 2011, in High-energy Emission from Pulsars and Their Systems, ed. N. Rea \& D. F. Torres (Berlin: Springer), 69

Górski, K. M., Hivon, E., Banday, A. J., et al. 2005, ApJ, 622, 759

Gotthelf, E. V., \& Halpern, J. P. 2008, ApJ, 681, 515

Grenier, I. A., Casandjian, J.-M., \& Terrier, R. 2005, Science, 307, 1292

Grondin, M.-H., Funk, S., Lemoine-Goumard, M., et al. 2011, ApJ, 738, 42

Hwang, U., Petre, R., \& Flanagan, K. A. 2008, ApJ, 676, 378

James, F., \& Roos, M. 1975, Comput. Phys. Commun., 10, 343

Kargaltsev, O., Pavlov, G. G., \& Wong, J. A. 2009, ApJ, 690, 891

Katagiri, H., Tibaldo, L., Ballet, J., et al. 2011, ApJ, 741, 44

Kerr, M. 2011, PhD thesis, Univ. Washington

King, I. 1962, AJ, 67, 471

Krause, J., Carmona, E., \& Reichardt, I. 2011, in Presented at the 2011 Fermi Symposium, Rome, Italy (available at http://www.slac.stanford.edu/econf/ C110509/)

Landi, R., de Rosa, A., Dean, A. J., et al. 2007a, MNRAS, 380, 926

Landi, R., Masetti, N., Bassani, L., et al. 2007b, ATel, 1047, 1

Markwardt, C. B., \& Ogelman, H. 1995, Nature, 375, 40

Matsumoto, H., Uchiyama, H., Sawada, M., et al. 2008, PASJ, 60, 163

Matsumoto, H., Ueno, M., Bamba, A., et al. 2007, PASJ, 59, 199

Mattox, J. R., Bertsch, D. L., Chiang, J., et al. 1996, ApJ, 461, 396

Mizukami, T., Kubo, H., Yoshida, T., et al. 2011, ApJ, 740, 78

Murphy, T., Mauch, T., Green, A., et al. 2007, MNRAS, 382, 382

Neronov, A., Semikoz, D. V., Tinyakov, P. G., \& Tkachev, I. I. 2011, A\&A, 526, A90

Nolan, P. L., Abdo, A. A., Ackermann, M., et al. 2012, ApJS, 199, 31

Paron, S., Dubner, G., Reynoso, E., \& Rubio, M. 2008, A\&A, 480, 439

Petre, R., Becker, C. M., \& Winkler, P. F. 1996, ApJ, 465, L43

Pollock, A. M. T. 1985, A\&A, 150, 339

Protassov, R., van Dyk, D. A., Connors, A., Kashyap, V. L., \& Siemiginowska, A. 2002, ApJ, 571, 545

Reynoso, E. M., Dubner, G. M., Goss, W. M., \& Arnal, E. M. 1995, AJ, 110,318

Reynoso, E. M., Green, A. J., Johnston, S., et al. 2003, MNRAS, 345, 671

Rodriguez, J., Tomsick, J. A., Foschini, L., et al. 2003, A\&A, 407, L41

Rowell, G., Horns, D., Fukui, Y., \& Moriguchi, Y. 2008, in AIP Conf. Ser. 1085, High Energy Gamma-ray Astronomy, ed. F. A. Aharonian, W. Hofmann, \& F. Rieger (Melville, NY: AIP), 241

Schwarz, G. 1978, Ann. Stat., 6, 461

Sreekumar, P., Bertsch, D. L., Dingus, B. L., et al. 1998, ApJ, 494, 523

Strong, A. W., \& Moskalenko, I. V. 1998, ApJ, 509, 212

Sugizaki, M., Mitsuda, K., Kaneda, H., et al. 2001, ApJS, 134, 77

Tanaka, T., Allafort, A., Ballet, J., et al. 2011, ApJ, 740, L51

Taylor, A. R., Gibson, S. J., Peracaula, M., et al. 2003, AJ, 125, 3145

Tomsick, J. A., Lingenfelter, R., Walter, R., et al. 2003, IAU Circ., 8076, 1

Torii, K., Kinugasa, K., Toneri, T., et al. 1998, ApJ, 494, L207

Vladimirov, A. E., Digel, S. W., Jóhannesson, G., et al. 2011, Comput. Phys. Commun., 182, 1156

Weekes, T. C., Cawley, M. F., Fegan, D. J., et al. 1989, ApJ, 342, 379

Weinstein, A. 2009, arXiv:0912.4492

Young, E. T., Lada, C. J., \& Wilking, B. A. 1986, ApJ, 304, L45

\footnotetext{
17 http://leejjoon.github.com/pywcsgrid2/

$18 \mathrm{http} / / /$ healpix.jpl.nasa.gov/
} 